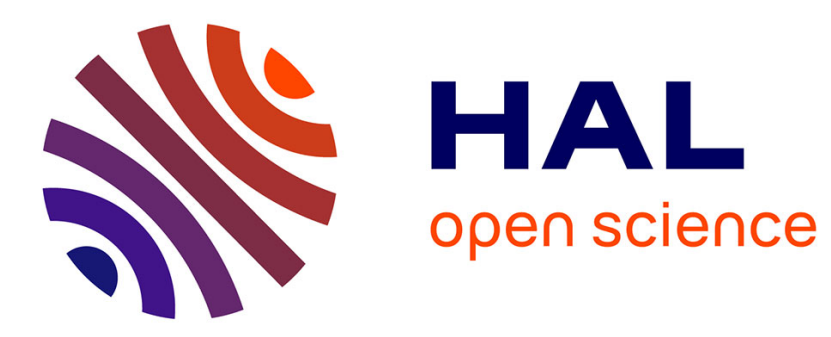

\title{
Les productions lamellaires dans l'Aurignacien de l'abri Pataud, Les Eyzies-de-Tayac (Dordogne)

\author{
Laurent Chiotti
}

\section{To cite this version:}

Laurent Chiotti. Les productions lamellaires dans l'Aurignacien de l'abri Pataud, Les Eyzies-deTayac (Dordogne). Gallia Préhistoire - Archéologie de la France préhistorique, 2003, 45, pp.113-156. 10.3406/galip.2003.2037 . hal-02353425

\section{HAL Id: hal-02353425 \\ https://hal.science/hal-02353425}

Submitted on 21 Jan 2020

HAL is a multi-disciplinary open access archive for the deposit and dissemination of scientific research documents, whether they are published or not. The documents may come from teaching and research institutions in France or abroad, or from public or private research centers.
L'archive ouverte pluridisciplinaire HAL, est destinée au dépôt et à la diffusion de documents scientifiques de niveau recherche, publiés ou non, émanant des établissements d'enseignement et de recherche français ou étrangers, des laboratoires publics ou privés.

\section{(ㅇ)(1) $\$$}

Distributed under a Creative Commons Attribution - NonCommercial - NoDerivatives| 4.0 


\title{
LES PRODUCTIONS LAMELLAIRES DANS L'AURIGNACIEN DE L'ABRI PATAUD, LES EyZIES-DE-TAYAC (DORDOGNE)
}

\author{
Laurent ChIOTTI*
}

Mots-clés. Aurignacien, lamelle, grattoir caréné, grattoir à museau, burin busqué, nucléus à lamelles, lamelle Dufour.

Résumé. Durant l'Aurignacien, diverses techniques ont été développées afin de produire des lamelles. Dans la séquence aurignacienne de l'abri Pataud (9 niveaux), ces différentes chaînes opératoires ont pu être mises en évidence et leur importance précisée pour chaque stade de l'Aurignacien. La production de lamelles à partir des grattoirs aurignaciens (carénés et à museau) est présente durant toute la séquence. Comme c'est généralement le cas, la production à partir des burins busqués se situe quasi exclusivement dans l'Aurignacien évolué, cependant, une chaîne opératoire identique a pu être décrite dans l'un des niveaux de l'Aurignacien ancien. Dans l'Aurignacien ancien, les petites lamelles qui sont produites à partir des grattoirs sont plutôt des lamelles rectilignes ou courbes, alors que dans l'Aurignacien

(II rolué elles sont essentiellement courbes et torses, qu'elles soient produites à partir des grattoirs ou des burins. Une production de lamelles de plus grandes dimensions à partir de nucléus prismatiques existe durant toute la séquence, même si elle est toujours faible. L'outillage amellaire est peu développé, à l'exception des lamelles Dufour du niveau 8 (Aurignacien évolué), et ce malgré la grande quantité te lamelles produite, ce qui pose la question de leur utilisation brute.

Key-words. Aurignacian, bladelet, carinate scraper, nose-shaped scraper, busqued burin, core bladelets, Dufour bladelet.

Abstract. During Aurignacian period, several techniques have been developped to produce bladelets. In the Aurignacian sequence of abri Pataud (9 levels) these lithic reduction sequences have been identified and their importance in each stage of the Aurignacian is specified. Bladelet production from Aurignacian scrapers (carinated and nose-shaped) appears in the whole sequence. As it is usually the case, the bladelet production from busqued burin is almost exclusively present in the evolved Aurignacian. However, a similar reduction sequence has been identified in one of the early Aurignacians levels. In the early Aurignacian, small bladelets producing from scrapers are rather straight or curved. At the opposite, the evolved Aurignacian bladelets, produced from scrapers and burins are mainly curved and twisted. Long bladelets are produced from prismatic cores during all the Aurignacian sequence, but always on a small scale production. Bladelet tools are underdevelopped, except Dufour bladelets in level 8 and so it raises the question of their unretouched use.

Palabras claves. Auriñaciense, laminitas, raspadores carenados, raspadores de hocico, buriles busqué, núcleos de laminitas, laminitas Dufour.

Resumen. Durante el Auriñaciense, se desarrollaron diversas técnicas a fin de producir laminitas. En la secuencia auriñaciense del abrigo Pataud (nueve niveles), dichas cadenas operatorias y su respectiva importancia fueron evidenciadas, en cada fase del Auriñaciense. La producción de laminitas a partir de raspadores auriñacienses (carenados o de hocico) se observa a lo largo de toda la secuencia. Como suele ocurrir, la producción a partir de buriles encorvados se sitúa casi exclusivamente durante el Aurinaciense evolucionado. Sin embargo, una cadena operatoria idéntica se halla en uno de los niveles del Auriñaciense antiguo. Durante el Auriñaciense antiguo, las pequeñas laminitas producidas con raspadores son más bien laminitas rectas o curvas, mientras que durante el Auriñaciense evolucionado, en mayoria son curvas y retorcidas - sean producidas con raspadores o con buriles -. Una producción

\footnotetext{
* FRE 2676 du CNRS, Muséum national d’histoire naturelle, abri Pataud, 20 rue dụ Moven Àge, F-24fi20 I.es Fyzies-de-Tayac. Mél : Ichiotti@mnhn.fr
} 
de laminitas de mayores dimensiones a partir de núcleos prismáticos es atestiguada a lo largo de toda la secuencia, aun que sea escasa.

El utillaje para laminitas no es muy desarrollado - salvo el caso de las laminitas Dufour del nivel 8 (Auriñaciense evolucionado) -

a pesar de la gran cantidad de laminitas producidas, lo cual induce que consideremos su uso sin retoque.

Situé dans le village des Eyzies-de-Tayac (Dordogne), au pied de la falaise calcaire qui domine le village d'une trentaine de mètres de hauteur, l'abri Pataud a été fouillé entre 1958 et 1964 par le professeur américain Hallam Leonard Movius.

Cet ancien abri-sous-roche, qui s'est avéré d'une richesse exceptionnelle, a livré une séquence stratigraphique de première importance pour la connaissance des débuts du Paléolithique supérieur en Périgord. La stratigraphie mise au jour (fig. 1) se compose de 9 niveaux aurignaciens (niveaux 14 à 6), de 4 niveaux gravettiens (niveaux 5 à 2) et d'un faible niveau solutréen (niveau 1), répartis sur une épaisseur de $9,25 \mathrm{~m}$ (Movius, 1977).

À l'issue des campagnes de fouilles, l'équipe francoaméricaine poursuivit son étude du matériel archéologique recueilli (Movius ed., 1975) jusqu'au début des années quatre-vingt.

À partir de 1986, le laboratoire de Préhistoire du Muséum national d'histoire naturelle a décidé de remettre le site en état et de reprendre une nouvelle étude pluridisciplinaire du matériel extrait lors de la fouille de H. L. Movius. C'est dans le cadre de ces nouvelles études que se place cette publication.

Les neuf niveaux aurignaciens de l'abri Pataud couvrent l'ensemble de la séquence aurignacienne classiquement reconnue en Périgord. Une étude technologique et typologique des industries en question nous a permis de préciser leurs attributions culturelles (Chiotti, 1999).

L'Aurignacien ancien est la période la mieux représentée (niveaux 14 à 9). Il s'agit d'un Aurignacien I, à sagaies à base fendue, tel qu'il a été défini à La Ferrassie (Savignac-de-Miremont, Dordogne) par D. Peyrony (Peyrony, 1934). Les industries de ces niveaux se caractérisent par une proportion importante de lames aurignaciennes, la quasi-absence des burins et la présence de grattoirs carénés. Les six niveaux correspondent à la même période malgré des différences notables dans les industries, différences qui semblent dues essentiellement à la durée et au mode d'occupation du site.

Il faut noter qu'il n'existe pas à l'abri Pataud d'équivalent de l'Aurignacien 0 défini par $\mathrm{H}$. Delporte
(Delporte, 1968), et ce malgré des dates relativement anciennes pour l'Aurignacien du Périgord (34250 $\pm 675 \mathrm{BP}$ pour la couche 14). Cependant, ceci n'est pas si surprenant au vu des nouvelles données qui tendent à remettre en cause l'existence de l'Aurignacien 0, tout au moins sur certains des sites où il avait été identifié (Bordes, 2000).

L'Aurignacien évolué, qui regroupe les stades II, III et IV définis par D. Peyrony à La Ferrassie (Peyrony, 1934), est représenté par les industries des niveaux 8 à 6 , qui peuvent chacune être rattachées aux différents stades.

Le niveau 8 a livré une industrie qui correspond à un Aurignacien II. Elle se caractérise par l'apparition des burins en quantité importante et la forte présence de grattoirs aurignaciens (surtout des grattoirs à museau) et de lamelles Dufour. Le caractère le plus étonnant de cette industrie est l'absence de burins busqués, alors que ces outils font généralement leur apparition, parfois en quantité importante, lors de ce stade II de l'Aurignacien du sud-ouest de la France.

Le niveau 7: Lower contenait un faciès plus rare en Périgord, qui est classé différemment selon les auteurs. Il s'agit d'une industrie avec une très forte proportion de burins $(34,89 \%)$, et en particulier de burins busqués $(12,54 \%)$. Selon D. de Sonneville-Bordes et F. Djindjian, les industries de ce type correspondent à un faciès particulier de l'Aurignacien II, que F. Djindjian dénomme Aurignacien IIr (Bouyssonie, SonnevilleBordes, 1957 ; Sonneville-Bordes, 1970 ; Djindjian, 1993), alors que selon la définition de P.-Y. Demars, il s'agit d'un Aurignacien III (Demars, 1994 ; Djindjian, 1993).

Les niveaux 7 : Upper et 6 contenaient des industries très comparables qui se caractérisent une fois encore par l'importance des burins, les burins busqués étant en quantité plus modérée. Ce sont des industries qui entrent dans la variabilité de l'Aurignacien IV, stade qui, rappelons-le, présente un polymorphisme important.

Le présent article va s'attacher à identifier les différents types de productions lamellaires présents dans toute la séquence aurignacienne de l'abri Pataud, pour l'ensemble des stades précédemment décrits. 


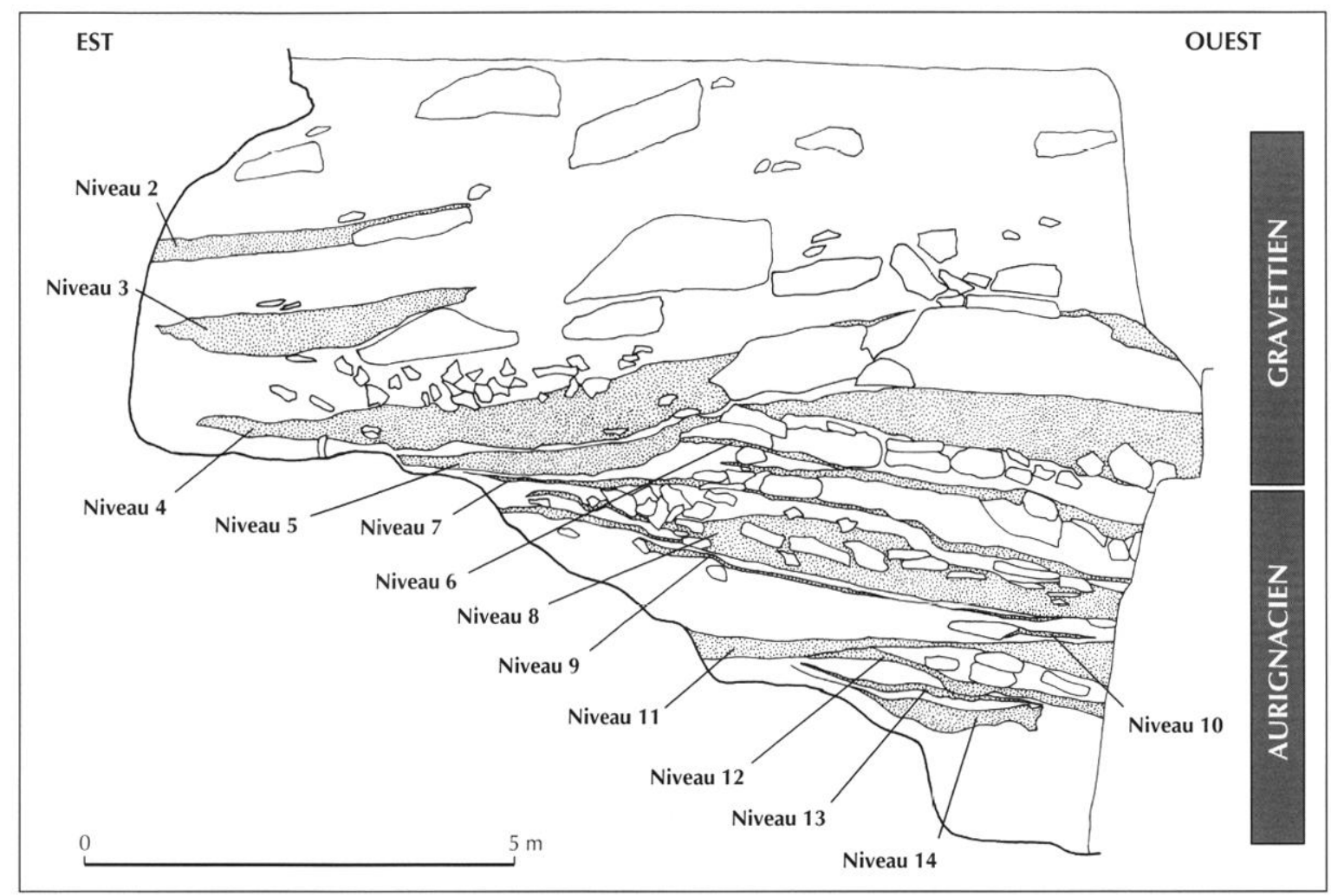

Fig. 1 - Coupe stratigraphique de l'abri Pataud.

\section{MÉTHODOLOGIE}

L'ensemble des lamelles des neuf niveaux aurignaciens de l'abri Pataud ont été prises en compte lors de cette étude. Différents caractères ont été observés.

\section{Les dimensions des lamelles}

La longueur et la largeur ont été mesurées au dixième de millimètre, la pièce étant orientée selon son axe technologique (fig. 2). La longueur a été mesurée uniquement pour les pièces entières. Dans le cas de pièces fragmentaires, seule la largeur a été prise en compte.

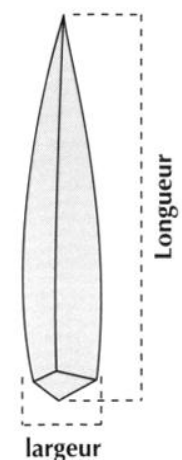

Fig. 2 - Méthode de mesure des dimensions (longueur et largeur) des lamelles.

\section{La courbure et la torsion}

La courbure est définie comme étant une concavité de la face d'éclatement de la pièce. La torsion peut, quant à elle, être définie comme un mouvement de vrille de l'extrémité distale par rapport à l'extrémité proximale de la pièce.

Ces aspects, observés pour toutes les lamelles, sont difficiles à mesurer et à quantifier. Il a donc simplement été noté s'ils sont présents ou absents, ce qui est estimé
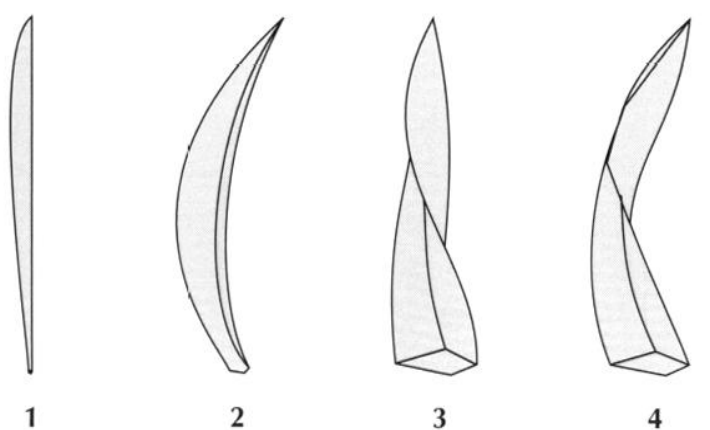

4

Fig. 3 - Catégories retenues lors de l'étude pour la description de la courbure et de la torsion des lamelles: 1, lamelles rectilignes; 2 , lamelles courbes; 3 , lamelles torses; 4 , lamelles courbes et torses. 


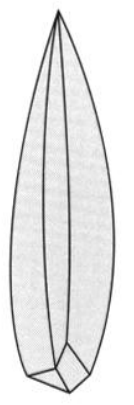

1

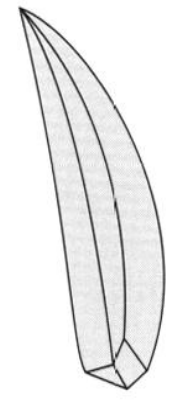

2

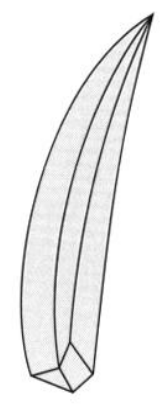

3
Fig. 4 - Catégories retenues lors de l'étude pour la description de l'aspect déjeté ou non des lamelles: 1, lamelles non déjetées; 2, lamelles déjetées vers la droite; 3, lamelles déjetées vers la gauche.

en posant la lamelle à plà sur sa face d'éclatement. Quatre catégories ont ainsi été définies (fig. 3) :

- des lamelles rectilignes;

- des lamelles courbes;

- des lamelles torses;

- des lamelles à la fois courbes et torses.

\section{L'aspect déjeté ou non déjeté des lamelles}

Certaines lamelles ne sont pas rectilignes et présentent une déviation de leur extrémité distale par rapport à l'axe technologique de la pièce. Cette déviation peut, selon les cas, se situer vers la droite ou vers la gauche. Comme pour le précédent, nous n'avons pas quantifié ce caractère, mais nous avons simplement noté sa présence ou son absence. Il a été estimé visuellement en posant la pièce sur du papier millimétré, à plat sur sa face d'éclatement, selon son axe technologique. Pour décrire ce caractère, nous avons retenu trois catégories (fig. 4) :

- des lamelles non déjetées ;

- des lamelles déjetées vers la droite ;

- des lamelles déjetées vers la gauche.

La combinaison de ces critères principaux nous a permis d'obtenir une représentation fidèle des différents types de lamelles, permettant ainsi, dans la majorité des cas, d'identifier le schéma de production dont elles sont issues.

D'autres caractères ont également été observés.

Sur l'ensemble des lamelles:

- matière première du support (silex sénonien local noir ou blond, silex du Bergeracois, silex jaspoïde, silex indéterminé...) ;

- présence ou absence de cortex ;
- position du cortex lorsque celui-ci est présent (en position proximale, distale ou latérale) ;

- état de fragmentation des pièces (entier, proximal, mésial ou distal).

Plus ponctuellement sur certains types de lamelles :

- présence ou absence d'une facette abrupte sur l'un des bords de la pièce ;

- présence ou absence des traces d'une encoche d'arrêt à l'extrémité distale de la pièce.

\section{TYPOLOGIE MORPHO-DIMENSIONNELLE DES LAMELLES}

Parmi les différents niveaux aurignaciens de l'abri Pataud, nous avons pu distinguer plusieurs types de lamelles. Ces types ont été différenciés en fonction du schéma opératoire dont les pièces sont issues.

Les deux principaux schémas généralement présents dans l'Aurignacien consistent en une production soit à partir d'outils-nucléus (pièces carénées, qu'il s'agisse de grattoirs ou de burins), soit à partir de nucléus prismatiques. Ces deux types de production ont pu être identifiés dans les niveaux 6 à 14 de l'abri Pataud.

\section{LES LAMELLES ISSUES DES PIÈCES CARÉNÉES}

Nous avons pu identifier trois types de lamelles différents correspondant toutes à ce schéma de production.

Lamelles de type A : petites lamelles dont la longueur est comprise entre $10 \mathrm{~mm}$ et $20 \mathrm{~mm}$ et la largeur entre $4 \mathrm{~mm}$ et $8 \mathrm{~mm}$. Ces lamelles sont généralement à la fois courbes et torses, mais elles peuvent parfois être seulement courbes ou plus rarement seulement torses. Elles sont le plus souvent déjetées.

Lamelles de type B : lamelles de plus grandes dimensions dont la longueur est comprise entre $25 \mathrm{~mm}$ et $40 \mathrm{~mm}$ et la largeur entre $8 \mathrm{~mm}$ et $12 \mathrm{~mm}^{1}$. Comme les

1. Lors de notre étude, nous avons séparé les lames des lamelles selon la définition de J. Tixier (1963), c'est-à-dire suivant une limite arbitraire de leur largeur fixée à $12 \mathrm{~mm}$. Cette définition reste acceptable pour les lamelles issues des nucléus prismatiques qui technologiquement sont similaires aux lames. Cependant, elle ne tient pas compte de la technologie. Pour ces pièces du type $B$, nous avons inclus des lamelles présen- 
lamelles de type A, elles sont le plus souvent courbes et torses et déjetées. Certaines d'entre elles conservent sur leur extrémité distale des traces d'aménagement de la carène du grattoir caréné dont elles sont issues (fig. 9, $\left.n^{\circ} 4\right)$.

Lamelles de type $\mathbf{C}$ : lamelles dont les caractéristiques dimensionnelles et morphologiques sont identiques à celles du type A, mais qui présentent en plus une facette abrupte, généralement positionnée sur leur bord droit. Ce type de lamelles provient en fait des burins aurignaciens, et la facette abrupte correspond à une partie de la face d'éclatement du support du burin, qui a été emportée lors du détachement de la lamelle.

Les trois types de lamelles (A, B et C) proviennent des pièces carénées aurignaciennes, mais ont une origine différente parmi ces dernières.

Les lamelles du type A peuvent avoir plusieurs origines différentes. Elles peuvent provenir soit des grattoirs à museau qui sont fréquents dans le niveau 8 , soit des grattoirs carénés qui sont présents en plus ou moins grande quantité dans tous les niveaux aurignaciens, soit enfin des burins aurignaciens (carénés, busqués, ou des Vachons) qui sont essentiellement présents dans le niveau 7 : Lower.

Comme nous l'avons déjà signalé, les lamelles du type B proviennent exclusivement des grattoirs carénés, et celles du type $\mathrm{C}$ des burins aurignaciens.

L'identification de l'origine technologique des lamelles de types $\mathrm{B}$ et $\mathrm{C}$ ne pose donc pas de problème important. En revanche, pour le type A, nous avons vu que l'origine pouvait être multiple: grattoirs carénés, grattoirs à museau ou burins aurignaciens. Dans les niveaux où ces différents types d'outils-nucléus sont présents, il est impossible de savoir de quel schéma de débitage proviennent les lamelles de type A. Nous parlerons alors de lamelles indifférenciées de type A. Cependant, dans certains niveaux, ils ne sont pas tous présents. Par exemple, dans le niveau 8, il n'y a pas de burin aurignacien, ce qui permet d'affirmer que les lamelles de type A proviennent exclusivement des grattoirs, qu'ils soient carénés ou à museau.

tant les mêmes caractéristiques morphologiques, mais dont la largeur est supérieure à la limite de $12 \mathrm{~mm}$. En effet, du fait de leur origine technologique (grattoirs carénés), ces pièces ne peuvent pas être considérées comme de vraies lames.

\section{LES LAMELLES ISSUES DES NUCLÉUS PRISMATIQUES}

Un type de lamelles différent des précédents est présent dans l'Aurignacien de l'abri Pataud, mais en quantité moins importante. Ces lamelles que nous dénommerons lamelles de type $\mathrm{D}$ sont des pièces dont la longueur est généralement comprise entre $20 \mathrm{~mm}$ et $40 \mathrm{~mm}$. Cependant cette donnée ne peut être considérée comme fiable car seules 17 pièces entières ont été identifiées pour l'ensemble des niveaux aurignaciens. La largeur, beaucoup plus fiable, est quant à elle comprise entre $8 \mathrm{~mm}$ et $12 \mathrm{~mm}$.

Il s'agit toujours de lamelles non déjetées. La plupart sont rectilignes, mais certaines sont courbes; en revanche, elles sont rarement torses ou courbes et torses.

Le fait que ces lamelles de type $\mathrm{D}$ soient issues de nucléus prismatiques ne pose pas de problème, en revanche leur place dans la séquence de débitage de ces nucléus est plus difficile à cerner. En effet, trois cas sont possibles :

- des lamelles provenant de petits nucléus prismatiques à lamelles stricto sensu;

- des lamelles provenant de la réduction de nucléus prismatiques à lames ;

- des lamelles intercalées dans des productions de lames.

\section{AUTRES LAMELLES}

À côté des deux origines principales décrites cidessus, nous avons également trouvé une petite série de lamelles plus irrégulières qui ne semblent pas issues d'un schéma de production particulier, et qui ont été classées comme des lamelles opportunistes.

Enfin, un certain nombre de lamelles n'ont pas pu être replacées dans l'un ou l'autre des schémas de production précédents, le plus souvent en raison de leur état de fracturation. Elles ont été classées dans une catégorie à part, celle des lamelles indéterminées.

\section{INVENTAIRE}

Les lamelles sont présentes dans tous les niveaux aurignaciens de l'abri Pataud, mais elles sont réparties en 
Tabl. I - Répartition des différents types de lamelles dans les principaux niveaux aurignaciens de l'abri Pataud.

\begin{tabular}{|c|c|c|c|c|c|c|c|c|c|}
\hline \multicolumn{2}{|c|}{ Niveau } & $\begin{array}{c}\text { Burin } \\
\text { aurignacien } \\
\text { (type C) }\end{array}$ & $\begin{array}{c}\text { Indifférencié } \\
\text { (type A) }\end{array}$ & $\begin{array}{c}\text { Grattoir caréné } \\
\text { ou à museau } \\
\text { (type A) }\end{array}$ & $\begin{array}{l}\text { Grattoir } \\
\text { caréné } \\
\text { (type B) }\end{array}$ & Indéterminé & $\begin{array}{c}\text { Nucléus } \\
\text { prismatique } \\
\text { (type D) }\end{array}$ & Opportuniste & Total \\
\hline \multirow{2}{*}{6} & $\mathrm{nb}$ & 5 & 64 & 0 & 68 & 70 & 35 & 17 & 259 \\
\hline & $\%$ & 1,93 & 24,71 & 0 & 26,25 & 27,03 & 13,51 & 6,56 & 100 \\
\hline \multirow{2}{*}{7 : Upper } & $\mathrm{nb}$ & 24 & 31 & 0 & 29 & 12 & 12 & 7 & 115 \\
\hline & $\%$ & 20,87 & 26,96 & 0 & 25,22 & 10,43 & 10,43 & 6,09 & 100 \\
\hline \multirow{2}{*}{7 : Lower } & $\mathrm{nb}$ & 49 & 111 & 0 & 85 & 38 & 31 & 18 & 332 \\
\hline & $\%$ & 14,76 & 33,43 & 0 & 25,60 & 11,45 & 9,34 & 5,42 & 100 \\
\hline \multirow{2}{*}{8} & $\mathrm{nb}$ & 5 & 0 & 738 & 171 & 174 & 30 & 31 & 1149 \\
\hline & $\%$ & 0,44 & 0 & 64,23 & 14,88 & 15,14 & 2,61 & 2,7 & 100 \\
\hline \multirow{2}{*}{11} & $\mathrm{nb}$ & 0 & 0 & 41 & 64 & 65 & 16 & 23 & 209 \\
\hline & $\%$ & 0 & 0 & 19,62 & 30,62 & 31,1 & 7,66 & 11 & 100 \\
\hline \multirow{2}{*}{12} & $\mathrm{nb}$ & 0 & 0 & 64 & 90 & 68 & 37 & 15 & 274 \\
\hline & $\%$ & 0 & 0 & 23,36 & 32,85 & 24,82 & 13,5 & 5,47 & 100 \\
\hline \multirow{2}{*}{13} & $\mathrm{nb}$ & 16 & 0 & 41 & 15 & 33 & 25 & 13 & 143 \\
\hline & $\%$ & 11,19 & 0 & 28,67 & 10,49 & 23,08 & 17,48 & 9,09 & 100 \\
\hline \multirow{2}{*}{14} & $\mathrm{nb}$ & 0 & 0 & 58 & 34 & 36 & 29 & 15 & 172 \\
\hline & $\%$ & 0 & 0 & 33,72 & 19,77 & 20,93 & 16,86 & 8,72 & 100 \\
\hline
\end{tabular}

quantités très inégales (tabl. I). L'ensemble des niveaux aurignaciens a livré un total de 2795 lamelles, mais le niveau 8 en contenait à lui seul près de la moitié ( 1149 pièces). Les autres niveaux sont tous nettement moins riches, avec un maximum de 332 lamelles dans le niveau 7 : Lower. Les éboulis séparant les niveaux d'occupation contiennent également des lamelles, mais toujours en très faibles quantités.

Pour la suite de cette étude, nous ne prendrons en compte que les niveaux contenant suffisamment de lamelles pour permettre une étude fiable, à savoir les niveaux 6, $7:$ Upper, $7:$ Lower, 8, 11, 12, 13 et 14.

\section{NIVEAUX 11, 12, 13 ET 14, AURIGNACIEN ANCIEN}

Dans les quatre niveaux en question, la production dominante est celle des lamelles provenant des grattoirs aurignaciens (types A et B, avec un total allant de 39,16\% pour le niveau 13 à $56,21 \%$ pour le niveau 12). Les lamelles de type $\mathrm{D}$ issues des nucléus sont peu représentées (de 7,66\% dans le niveau 11 à $17,48 \%$ dans le niveau 13). Quant aux lamelles opportunistes, elles ne dépassent jamais $11 \%$ (maximum dans le niveau 11).
Il faut noter une particularité importante du niveau 13 : la présence de lamelles de type $C$ issues de burins, qui sont totalement absentes dans les autres niveaux de l'Aurignacien ancien.

\section{PRODUCTION LAMELLAIRE DES GRATTOIRS CARÉNÉS ET À MUSEAU}

\section{LES GRATTOIRS-NUCLÉUS}

Les grattoirs aurignaciens sont représentés par un total de 79 pièces dans les quatre niveaux de l'Aurignacien ancien étudiés, soit treize dans le niveau 14 , deux dans le niveau 13 , vingt-quatre dans le niveau 12 et quarante dans le niveau 11 (fig. 5 et 6 ). Malgré le nombre de pièces qui peut paraître relativement important, cela ne représente qu'une très faible partie de l'outillage de ces niveaux: respectivement $7,34 \% ; 2,41 \% ; 6,20 \%$ et $4,10 \%$.

Les plus nombreux sont les grattoirs carénés avec 42 pièces, dont 4 doubles et 7 atypiques. Il y a également 13 grattoirs à museau épais, 16 grattoirs sur éclats épais et 8 grattoirs à épaulement.

La grande majorité de ces grattoirs (52 pièces) est réalisée sur des éclats. Il y en a également 20 sur des 

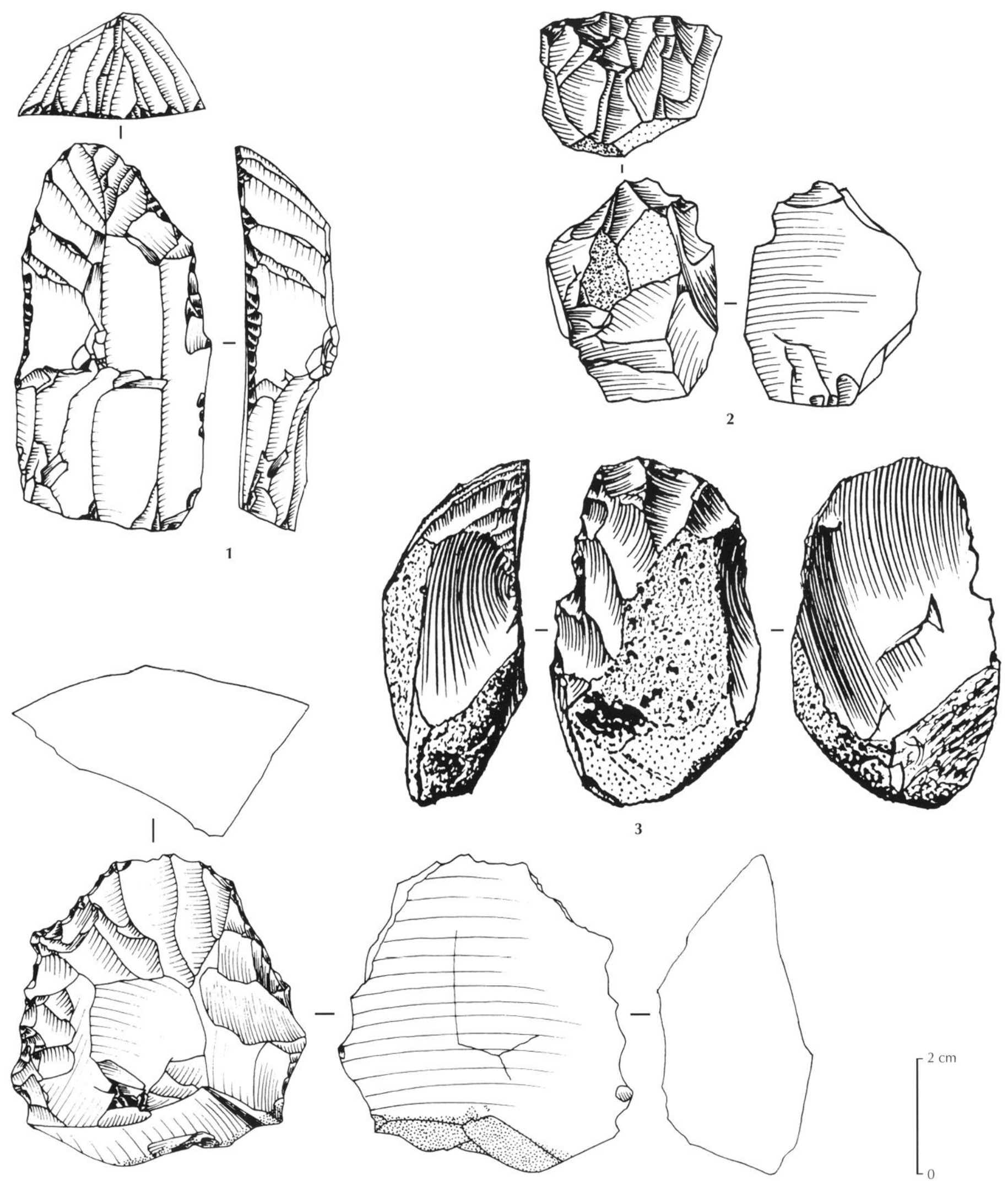

Fig. 5 - Crattoirs carénés el à museau de l'Aurignacien ancien : 1, 4, niveau $11 ; 2$, niverau $12 ; 3$, niveau 14 (dessins : 1, 2, 4, I. C.hivuli ; 3, M. Dauvois). 

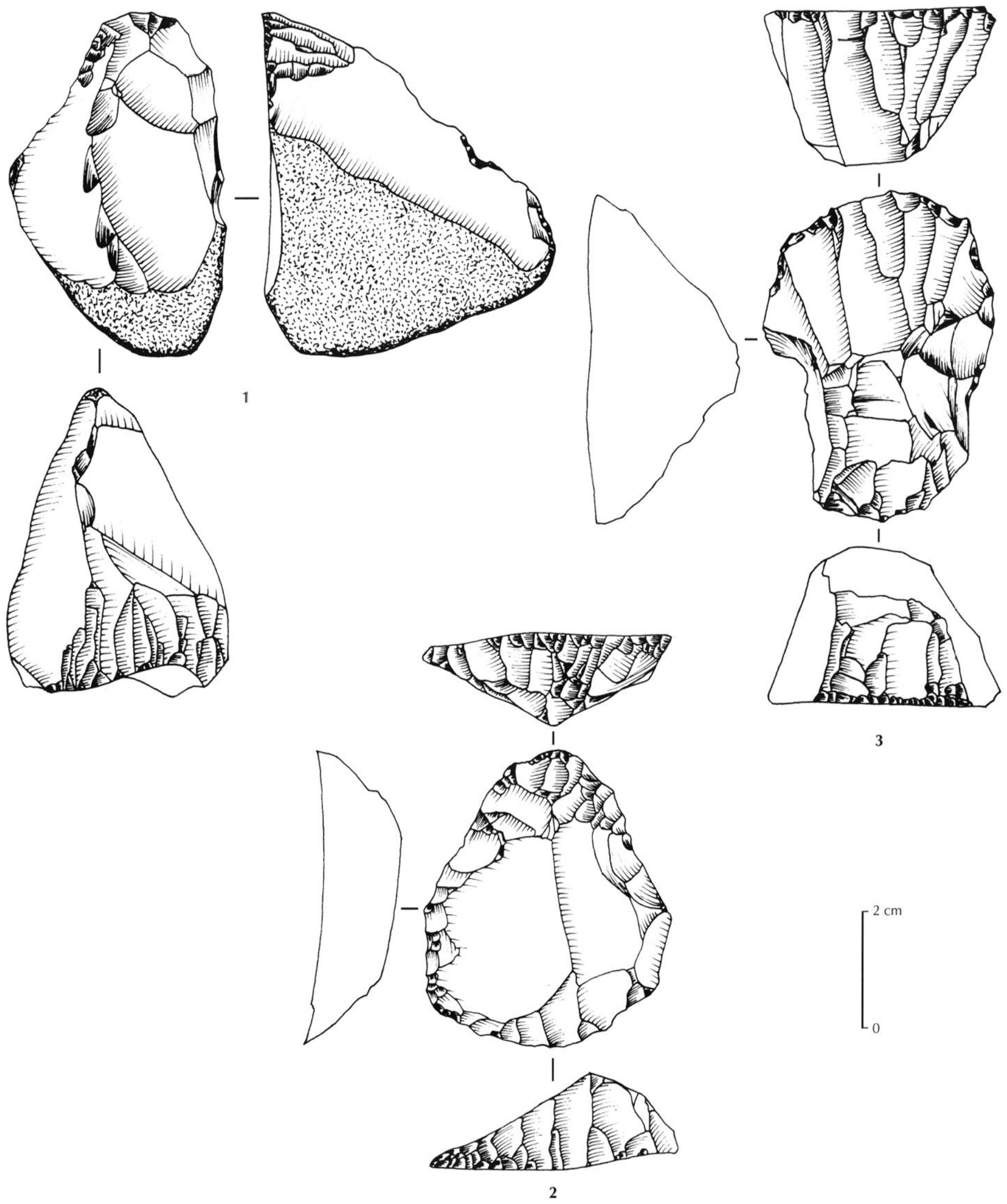

Fig. 6 - Grattoirs carénés et à museau de l'Aurignacien ancien : 1, niveau $12 ; 2,3$, niveau 14 (dessins L. Chiotti). 
blocs, et seulement 7 sur des lames. Les supports semblent avoir été choisis essentiellement en fonction de leurs dimensions. En effet, aussi bien les éclats que les lames choisis ont des dimensions nettement supérieures à la moyenne des supports produits dans ces industries (en particulier en ce qui concerne l'épaisseur). Les blocs, quant à eux, ont été peu transformés (ils sont souvent très corticaux), et ont donc été choisis en fonction de leurs dimensions et d'une forme appropriée.

Ces grattoirs aurignaciens présentent généralement très peu de macrotraces d'utilisation : seuls seize d'entre eux ont de très faibles esquillements sur la face inférieure qui partent généralement du front (quatre dans le niveau 14, un dans le niveau 13 et onze dans le niveau 11).

L'Aurignacien ancien a également livré des pièces identifiées par différents auteurs (Aubry et al., 1995 ; Lucas, 1997 ; Chiotti, 2000) comme étant des ravivages de grattoirs carénés, permettant de maintenir une encoche sur le côté du front. Elles sont au nombre de 9 : une dans le niveau 14, six dans le niveau 12 et deux dans le niveau 11.

\section{LeS lamelles DE TYPES A ET B}

Les lamelles provenant des grattoirs peuvent être séparées en deux groupes, essentiellement sur la base de leurs dimensions. Le premier groupe comprend des lamelles issues exclusivement de grattoirs carénés (type B) et le second des lamelles qui peuvent provenir soit de grattoirs carénés, soit de grattoirs à museau (type A).

Du point de vue de la répartition entre les deux types de lamelles, le comportement paraît différent entre les niveaux les plus anciens (14 et 13) et les plus récents (12 et 11).

Dans les premiers, ce sont les lamelles de type A qui dominent (tabl. I) sur celles de type B. Pour les niveaux 12 et 11 , le comportement est inversé, avec une dominance des lamelles de type B. Cependant, nous avons tendance à ne pas attacher une grande importance à ces différences de comportement en raison de la représentativité des échantillons étudiés. En effet, pour les niveaux 13 et 14 , une partie importante du matériel

2. À la fin des fouilles du professeur H. L. Movius, le matériel archéologique a été rangé dans des sacs en toile, et stocké dans une cave pré- lithique n'a pas été retrouvée lors des nouvelles études 2 (Chiotti, 1999), ce qui rend peu fiable les données sur ces niveaux, contrairement aux autres niveaux pour lesquels la quasi-totalité du matériel a pu être étudiée.

Sur les nuages de points représentant les pièces entières (fig. 7), les lamelles de type A sont toujours très regroupées (pour les quatre niveaux), alors que les lamelles de type $\mathrm{B}$ forment au contraire des nuages plus dispersés. De même, sur les histogrammes de largeurs prenant en compte la totalité des pièces (fig. 8), les lamelles de type A forment un pic nettement plus étroit que celles de type $B$.

La faible amplitude des largeurs ainsi que la faible dispersion des rapports longueur/largeur mettent en évidence la standardisation des dimensions des lamelles de type A provenant soit des grattoirs carénés, soit des grattoirs à museau. Au contraire, il ne semble pas y avoir de standardisation pour les lamelles de type B.

Ces dernières (fig. 9, nos 1-6) sont en majorité des pièces non déjetées $(46,34 \%$ de l'effectif des quatre niveaux). Les pièces déjetées le sont à peu près autant vers la gauche $(23,90 \%)$ que vers la droite $(20,00 \%)$. La majorité de ces lamelles sont courbes et torses (56,93\%). Les autres sont soit courbes $(22,77 \%)$, soit torses $(15,35 \%)$. En revanche, il n'y a quasiment pas de lamelles rectilignes.

Ce sont en fait les lamelles courbes et torses qui dominent, aussi bien parmi les pièces déjetées que non déjetées. Cependant, parmi les lamelles non déjetées, il y a également beaucoup de lamelles courbes, ce qui n'est pas le cas parmi celles qui sont déjetées.

Dans l'ensemble, nous avons pu constater l'existence d'une grande variabilité de formes au sein des lamelles de type $B$, ce qui est cohérent avec les fortes variations observées lors de l'étude des dimensions.

Techniquement, il s'agit des premières lamelles qui interviennent à la suite des phases de mise en forme, ou de réaménagement de la table de débitage. Pour la majorité d'entre elles, elles se situent sur les parties latérales du front. Elles ont souvent un caractère torse qui provient du fait qu'en partie distale l'axe de leur détachement est fréquemment dévié par la présence

sentant un fort taux d'humidité. Lors de la reprise des collections en 1986, une partie des sacs s'était dégradée et les informations concernant ceux-ci étaient perdues. Bien que cela ne concerne qu'une faible part du matériel extrait des fouilles, il s'avère qu'une partie importante du matériel lithique des niveaux 13 et 14 est dans ce cas. 

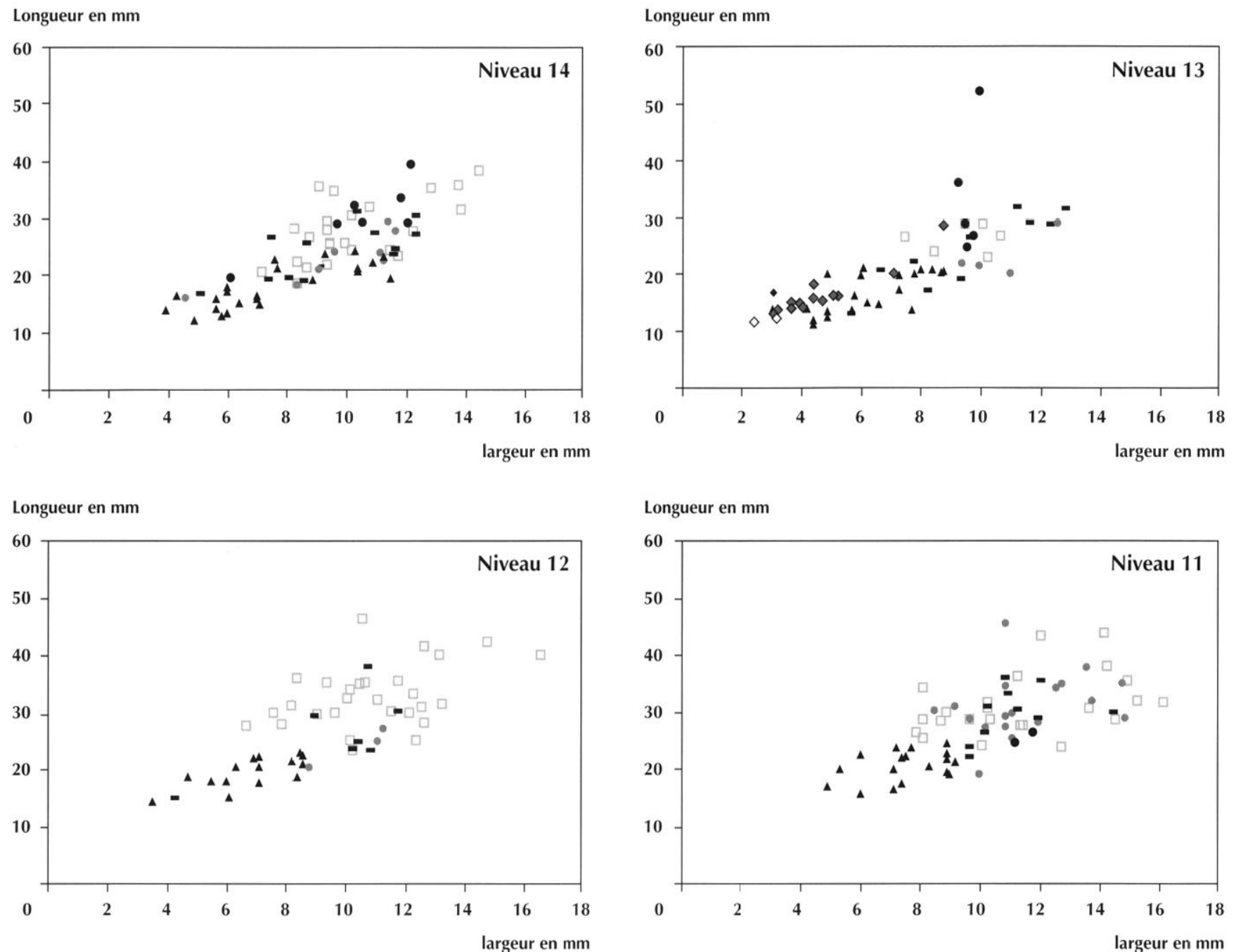

Longueur en mm

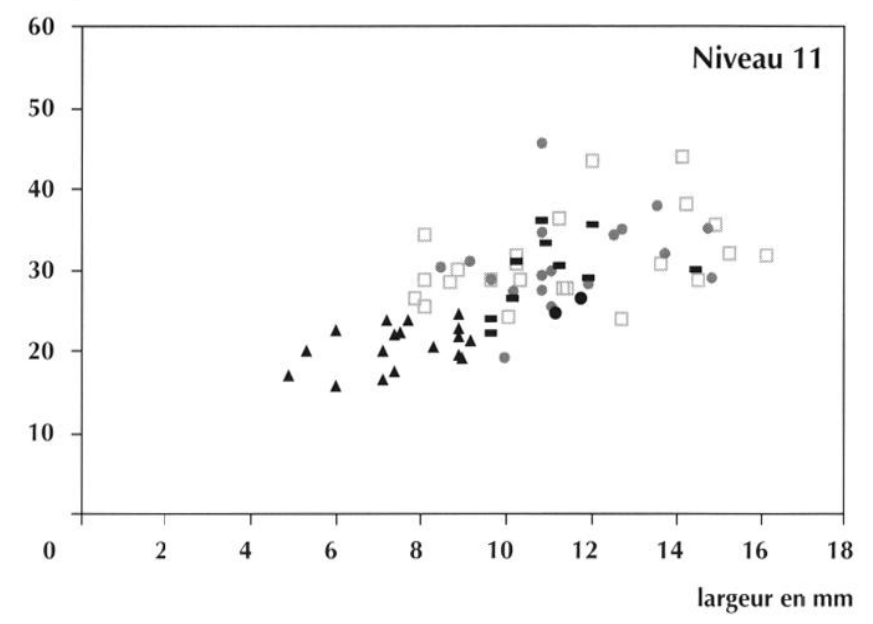

$$
\begin{array}{lll}
\square \text { lamelles des grattoirs carénés (type B) } & \bullet \text { lamelles indéterminées } & \bullet \text { lamelles des nucléus (type D) } \\
\Delta \text { lamelles des grattoirs carénés ou à museau (type A) } & - \text { lamelles opportunistes } & \diamond \text { lamelles des burins (type C) } \\
& & \diamond \text { négatifs de lamelles sur les biseaux des burins }
\end{array}
$$

Fig. 7 - Diagrammes présentant les rapports longueur/largeur des différents types de lamelles entières, dans les niveaux de l'Aurignacien ancien (niveaux 11, 12, 13 et 14).

lamelles des grattoirs carénés (type B)

nombre de pièces

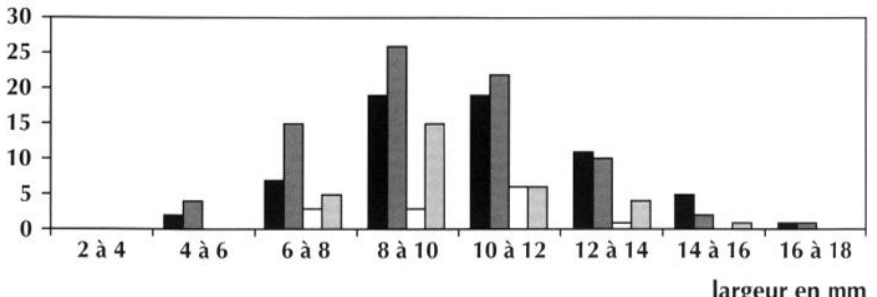

lamelles des grattoirs carénés et/ou à museau (type A)

nombre de pièces

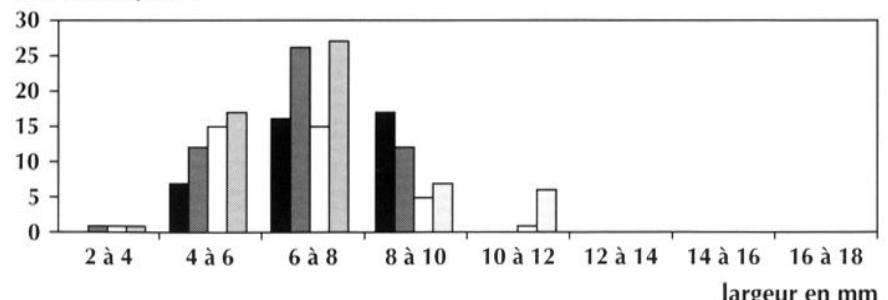

niveau $11-\square$ niveau 12

$\square$ niveau $13 \quad \square$ niveau 14

Fig. 8 - Histogrammes présentant les largeurs des lamelles provenant des grattoirs carénés (type B) et des grattoirs carénés el/ou à museau (type A) dans les niveaux d'Aurignacien ancien (niveaux $11,12,13$ et 14). 

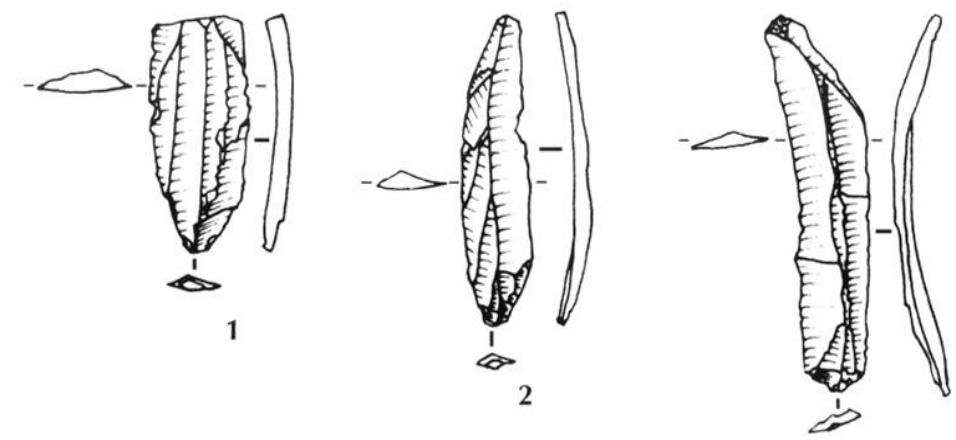

3

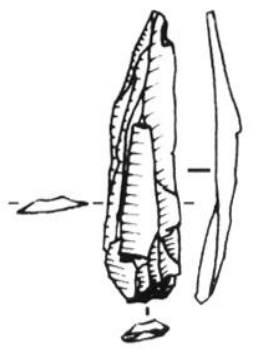

6

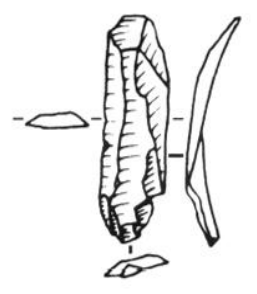

11

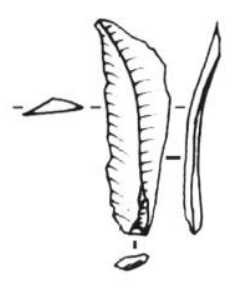

7

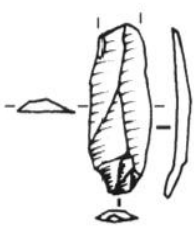

12

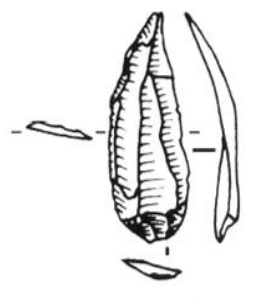

8

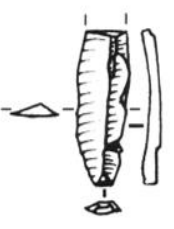

13
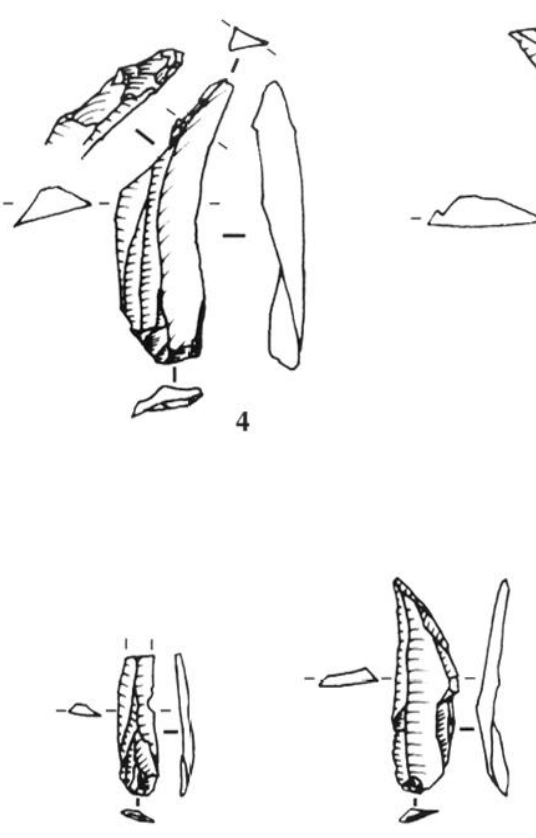

10

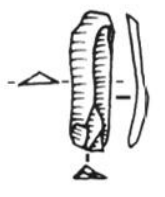

14

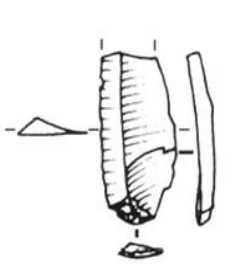

15

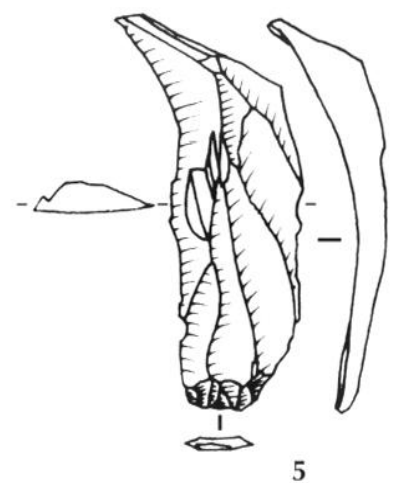

5

Fig. 9 - I.amelles issues des grattoirs de l'Aurignacien ancien : 1-6, lamelles de type B; 7-11, lamelles de type A courbes et torses; 12-15, lamelles de type B rectilignes ou courbes $(6-8,12:$ niveau $11 ; 1-3,9,10,13:$ niveau $12 ; 4,14,15:$ niveau $13 ; 5,11:$ niveau 14) (dessins L. Chiotti).

d'une " crête " sur la partie supérieure du grattoir (Bon, 2000). Elles portent parfois des traces de cette "crête " sur leur extrémité distale (fig. $9, \mathrm{n}^{\circ} 4$ ).

Parmi les lamelles de type A, il y a une forte majorité de pièces non déjetées $(59,11 \%$ de l'effectif des quatre niveaux). Le reste est constitué de 12,81\% de lamelles déjetées à gauche et 18,72\% déjetées à droite. Comme pour les lamelles de type B, il n'y a donc pas de latéralisation. Du point de vue de la courbure et de la torsion, la majorité des pièces présente en même temps les deux critères : courbes et torses $(51,47 \%)$. Il y a également une forte proportion de lamelles courbes $(29,90 \%)$. Au contraire, il y a très peu de lamelles torses $(9,80 \%)$ et rectilignes $(6,37 \%)$.

En définitive, parmi les lamelles de type A, deux groupes peuvent être distingués :
- des lamelles courbes et torses dont plus de la moitié

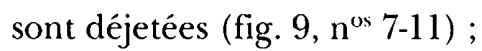

- des lamelles rectilignes qui ne sont quasiment jamais déjetées (fig. 9, nos 12-15).

Ces deux groupes correspondent en fait à un positionnement différent sur la surface de débitage des grattoirs-nucléus. Les lamelles courbes et torses sont débitées plutôt sur les côtés des fronts de grattoirs, alors que les lamelles courbes ou rectilignes sont débitées sur le centre du front. C'est cette position qui explique le fait que les premières soient en partie déjetées alors que les secondes ne le sont pas.

Du point de vue de leurs dimensions, les deux groupes ainsi identifiés correspondent, en revanche, à un même gabarit de lamelles puisqu'ils n'avaient pas du tout été mis en évidence lors de l'étude des dimensions. 
En conclusion, tous ces éléments tendent à montrer que ce sont les petites lamelles de type A qui étaient recherchées, les grandes, de type $B$, étant plutôt des phases de préparation. La variabilité de ces dernières peut alors s'expliquer par les différences de dimensions des blocs à mettre en forme afin de pouvoir débiter des petites lamelles ayant toujours le même gabarit dimensionnel.

Deux types principaux de lamelles ont donc pu être identifiés au sein de la production des grattoirs aurignaciens : des lamelles de type B, non standardisées, et des lamelles de type A comprenant deux groupes: des lamelles à la fois courbes et torses et des lamelles qui sont soit courbes, soit rectilignes.

Ces trois formes correspondent à trois phases successives du schéma de débitage des grattoirs-nucléus. Elles sont encore visibles sur certains fronts de grattoirs dans leur état d'abandon.

Les lamelles de type $\mathrm{B}$ correspondent à la préparation des parties latérales du front. Leurs dimensions sont très variables du fait de la diversité de formes et de tailles des supports des grattoirs. Elles ont un but de mise en forme ou d'entretien de la carène et du cintre de la surface de débitage afin que celle-ci ait la forme et les dimensions appropriées pour l'obtention des deux autres formes de lamelles.

Les lamelles de type A interviennent après le débitage des précédentes. Celles qui sont courbes et torses sont débitées de part et d'autre de la partie centrale du front ; les lamelles déjetées étant situées plus sur l'extérieur et les non déjetées plus vers le centre. Enfin, celles qui sont courbes ou rectilignes sont débitées sur le centre du front.

Cette séquence de production de lamelles peut se répéter plusieurs fois sur un même grattoir-nucléus, suivant les dimensions du bloc utilisé.

La question qui se pose après l'identification de ce schéma de débitage est de savoir, parmi ces formes de lamelles, laquelle était recherchée.

Les grandes lamelles de type B qui ne présentent aucune standardisation semblent n'être que des lamelles de préparation. En revanche, les deux formes de petites lamelles plus standardisées peuvent constituer le but de ce débitage.

Deux hypothèses peuvent être posées :

- soit ce sont les lamelles courbes et rectilignes qui sont recherchées, et dans ce cas les lamelles courbes et torses ne sont qu'une préparation des parties latérales du front ;

- soit ce sont les lamelles courbes et torses qui sont recherchées, et dans ce cas les lamelles courbes et rectilignes sont détachées par la suite sur le centre du front, afin de permettre la poursuite du débitage évitant ainsi le développement d'un museau trop prononcé.

Nous ne pouvons pas, dans l'état actuel de nos recherches, trancher de façon certaine entre ces deux hypothèses, cependant les fronts de grattoirs nous apportent un argument en faveur de la première. En effet, dans leur état d'abandon, ceux-ci présentent souvent un ou plusieurs négatifs de lamelles rectilignes ou courbes sur le centre. L'enlèvement de ces lamelles avant l'abandon n'était pas nécessaire si ce n'était pas celles-ci qui étaient recherchées.

D'autre part, la comparaison avec d'autres sites est également en faveur de la recherche de lamelles non torses. En effet, le schéma que nous avons identifié semble correspondre à celui mis en évidence par F. Lebrun-Ricalens dans l'Aurignacien ancien de Hui à Beauville (Lot-et-Garonne) ${ }^{3}$, si ce n'est que, contrairement à nous, l'auteur a pu identifier trois tailles de lamelles différentes, les deux plus grandes étant les produits de phases de préparation destinées à l'obtention des plus petites, qui sont des lamelles rectilignes ou courbes. De plus, J.-G. Bordes a également mis en évidence une volonté de produire des lamelles non torses sur les grattoirs de plusieurs sites : Caminade à Sarlat-laCanéda, Corbiac Vignoble 2 près de Bergerac (Dordogne), Le Piage à Fajoles (Lot), dans l'Aurignacien ancien (Bordes, à paraitre).

\section{PRODUCTION LAMELLAIRE DES BURINS AURIGNACIENS}

Les niveaux de l'Aurignacien ancien en Périgord contiennent généralement une très faible proportion de burins. L'Aurignacien de l'abri Pataud n'échappe pas à ce schéma, puisque seuls 27 burins ont été retrouvés dans l'ensemble des quatre niveaux principaux (deux dans le

\footnotetext{
3. F. L.e Brun-Ricalens, Modalités et variabilité de quelques productions lamellaires sur le site de plein air d'Hui (Beauville, Lot-et-Garonne, France), communication lors du XIV“ Congrès de l'UISPP, Liège, 2-9 sept. 2001, dans le cadre d'un colloque intitulé "Les productions lamellaires attribuées à l'Aurignacien : chaînes opératoires et perspectives techno-culturelles ».
} 


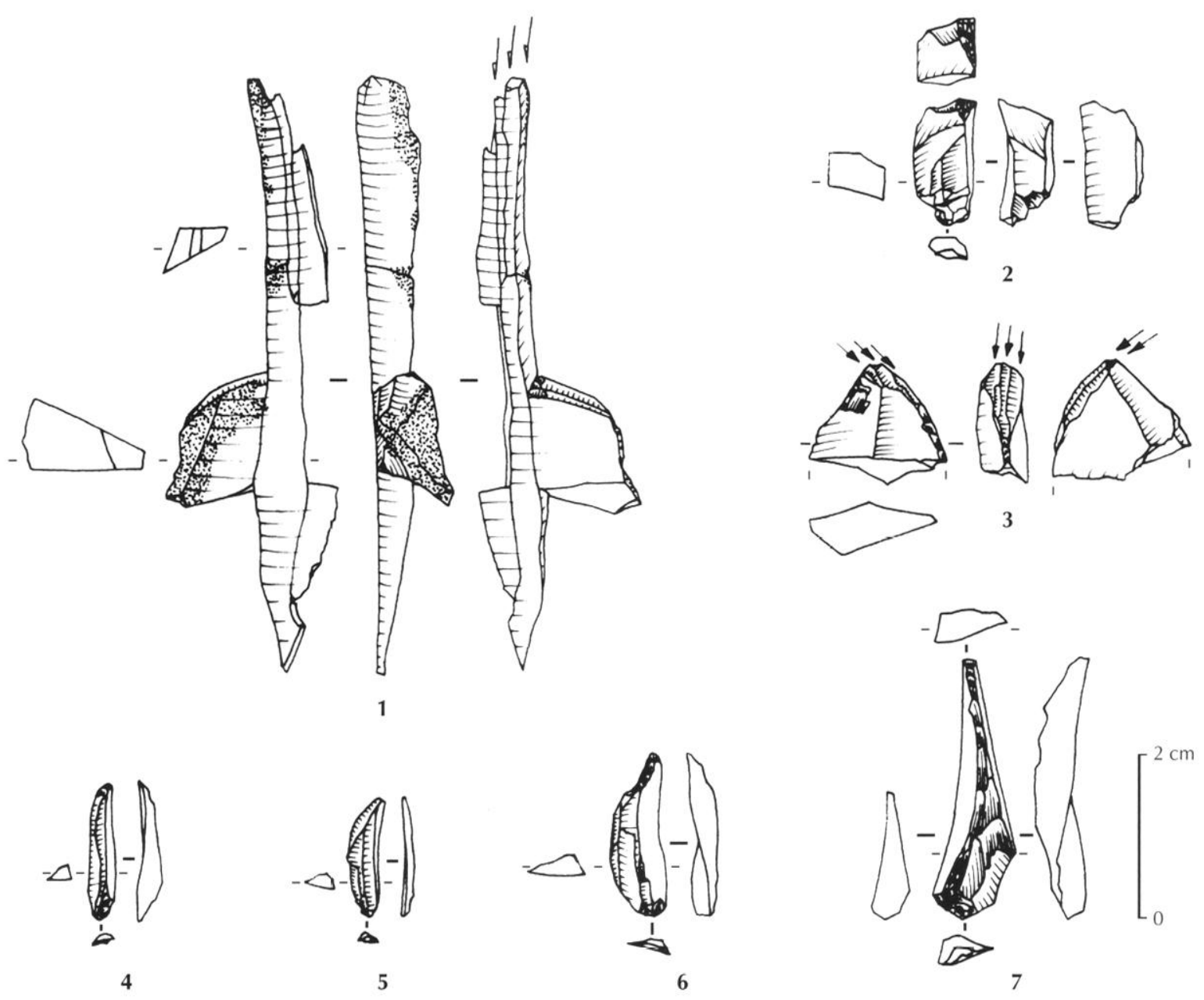

Fig. 10 - Éléments de la chaine opératoire de production de lamelles à partir des burins du niveau $13: 1$, remontage d'un burin-nucléus et de ses chutes; 2 , pièce de ravivage de la table de débitage; 3 , burin-nucléus; 4-6, lamelles de type $C ; 7$, lamelle permettant l'entame du débitage (dessins L. Chiotti).

niveau 14 ; huit dans le niveau 13 ; sept dans le niveau 12 et dix dans le niveau 11).

Malgré le faible nombre de burins, nous avons pu identifier, dans la couche 13 , une production de lamelles à partir des burins. L'identification de cette chaine opératoire a été possible grâce à la réalisation d'un remontage. Elle s'avère très proche de celle des burins busqués connue dans l'Aurignacien évolué.

\section{LES BURINS-NUCLÉUS}

Parmi les huit burins du niveau 13, deux peuvent être rattachés à cette production en tant que nucléus. Ils sont tous deux de petites dimensions $(15 \mathrm{~mm} \times 18 \mathrm{~mm}$ x $7 \mathrm{~mm})$ et $(17 \mathrm{~mm} \times 14 \mathrm{~mm} \times 10 \mathrm{~mm})$ car très fragmentaires, cassés immédiatement en arrière du biseau.
Dans les deux cas, il s'agit de fractures en flexion (l'une des deux est même une fracture en languette) qui évoquent des fractures d'utilisation, ce qui pourrait être un argument en faveur d'un usage de ces pièces comme burins après le débitage des lamelles. De plus, l'un de ces burins (fig. $10, n^{\circ} 3$ ) porte sur la face supérieure une retouche issue du négatif de la chute du pan primaire, qui ne semble pas liée au débitage des lamelles. Cette retouche nette, mais néanmoins de faible ampleur, est très certainement liée à une utilisation de la pièce postérieure au débitage des lamelles, et pourrait correspondre à des traces d'utilisation. Le biseau de ce même burin porte des esquillements qui s'étendent sur le négatif de chute de burin servant de plan de frappe ; ces esquillements semblent également liés à une utilisation. 
Malgré leur position dans l'Aurignacien ancien, ces deux burins sont typologiquement identiques à des burins busqués. Ils présentent toutes les caractéristiques de ces derniers :

- un pan primaire destiné à servir de plan de frappe pour le débitage des lamelles (sur l'un d'entre eux, ce plan a visiblement subi un ravivage) ;

- un pan secondaire formé de négatifs de chutes de burin arquées ;

- les traces d'une encoche d'arrêt à l'extrémité distale des négatifs lamellaires.

Chacun de ces burins présente sur son biseau un seul négatif de lamelle entier.

\section{LES LAMELLES DE TYPE C}

Certaines lamelles issues du débitage de ces burins busqués ont pu être identifiées. Elles sont relativement peu nombreuses ( 16 lamelles), mais étant donné la faible quantité de pièces dans cette couche, cela représente quand même $11,19 \%$ de la totalité des lamelles. Il s'agit de lamelles de type $\mathrm{C}$ présentant une petite facette abrupte sur l'un de leurs bords (fig. 10, $\mathrm{n}^{\text {os }} 4-6$ ). Ces lamelles sont très semblables à celles issues des burins busqués, identifiées dans les couches de l'Aurignacien évolué. Onze d'entre elles présentent des traces d'une retouche de préparation sur leur extrémité distale, qui correspondait probablement à l'encoche d'arrêt des lamelles/chutes de burins. L'angle de la facette est généralement un peu moins abrupt en partie proximale.

Ce sont en grande majorité des lamelles courbes et torses (13 pièces sur 16), le reste étant constitué de deux pièces courbes et d'une pièce rectiligne. Elles sont fortement latéralisées : la facette abrupte se situe sur le bord droit pour 13 lamelles sur 16. De plus, parmi ces seize lamelles, neuf sont déjetées à droite alors qu'une seule est déjetée à gauche. Les six autres sont rectilignes.

Le rapport longueur/largeur présente une très faible dispersion (fig. 7), révélant ainsi un gabarit très standardisé. À l'exception de deux pièces de dimensions plus importantes, ce sont les lamelles les plus petites du niveau 13. Les deux négatifs de lamelles présents sur les biseaux de burins ont des dimensions qui sont encore inférieures à celles de ces lamelles. Étant donné le nombre relativement faible de ces pièces, et au vu du remontage effectué (cf. infra), il est probable que nous ne soyons en présence que d'une partie de la production.
Les très faibles dimensions des lamelles et des négatifs sur les burins laissent penser qu'une partie importante des lamelles issues de cette chaîne opératoire n'a pas été récoltée lors de la fouille qui, rappelons-le, s'est déroulée dans les années soixante, et ne comportait la plupart du temps qu'un tamisage à sec.

Il faut également préciser qu'une partie des lamelles issues de cette chaîne opératoire n'a probablement pas été identifiée. En effet, celles qui sont débitées du côté de la surface supérieure du burin ne présentent pas la facette abrupte (qui n'est autre qu'une partie de la face d'éclatement du support de ce burin) qui nous a permis de les identifier. Dans ce cas, elles sont identiques aux lamelles de type A provenant des grattoirs aurignaciens et en particulier des grattoirs à museau. Cependant, nous avons vu que les lamelles issues de ces burins sont généralement plus petites. Or, il n'y a que très peu de pièces correspondant à ces dimensions parmi les lamelles de type $\mathrm{A}$, ce qui nous incite à penser qu'il n'y a probablement que très peu de mélange.

L'un des deux burins a pu être remonté avec une partie des chutes constituant le pan primaire, destiné à constituer le plan de frappe (fig. $10, \mathrm{n}^{\circ} 1$ ). Ce remontage met en évidence l'existence d'une très forte réduction de la lame utilisée comme nucléus. Le point de percussion de la dernière chute à l'origine du plan de frappe se situe à $38 \mathrm{~mm}$ de la surface de débitage dans son état d'abandon. Cette réduction très importante de la lame vient confirmer le rôle de nucléus de ces burins busqués. D'autre part, cette réduction confirme également le fait qu'une partie importante des petites lamelles n'a pas été récoltée à la fouille. En effet, pour toute la séquence de débitage de ce burin, nous n'avons retrouvé qu'une seule lamelle dans la même matière.

Nous avons également retrouvé deux pièces provenant d'autres burins (non retrouvés), qui constituent des étapes particulières de cette chaîne opératoire : une lamelle initiant le débitage et un ravivage de la table de débitage.

La première de ces pièces est l'une des petites lamelles courbes et torses présentant une facette abrupte, située sur le bord gauche. Cette lamelle porte une retouche sur toute la longueur de l'arête entre la facette abrupte et la face supérieure (fig. 10, n 7 ). Il s'agit d'une retouche qui équivaut à une « légère troncature " jouant le rôle de " crête " destinée à la mise en forme de la carène de la future table de débitage 
(Le Brun-Ricalens, Brou, sous presse). En partie proximale de la lamelle, la retouche est abrupte et écailleuse, constituant une vraie troncature. Elle se réduit en direction de l'extrémité distale, jusqu'à devenir une retouche marginale semi-abrupte.

La seconde pièce est un ravivage de la table de débitage. Il s'agit des pièces décrites par F. Le BrunRicalens et L. Brou comme « des lamelles volontairement outrepassées, souvent plus épaisses dont le profil de la ligne dorsale s'infléchit brutalement en ligne brisée ". C'est une lamelle qui emporte la totalité de la table de débitage du burin (fig. $10, \mathrm{n}^{\circ}$ 2). Ce type de pièce a déjà été décrit par M. Otte au Trou du Renard à Furfoo\% (Belgique), sous le terme de "fronts de burins carénés" (Otte, 1979) et F. Le Brun-Ricalens et L. Brou à Thèmes (Cézy, Yonne) (Le Brun-Ricalens, Brou, sous presse).

Dans ce cas précis, ce ravivage est intervenu à la suite d'une série de rebroussés qui rendaient l'ancienne table de débitage totalement inutilisable. L'extrémité distale de cette lamelle porte une retouche sur toute sa longueur, ce qui semble indiquer que les traces de retouche visibles sur l'extrémité de certaines lamelles ne correspondent pas à une encoche, mais plutôt à une retouche continue du bord.

Selon Le Brun-Ricalens et Brou, la convexité du carénage peut également être entretenue par des néocrêtes partielles ou totales. Nous avons retrouvé une petite lamelle qui porte des traces d'aménagement de type crête le long de la facette abrupte semblant correspondre à ce type d'aménagement. Cependant, cette pièce peu caractéristique ne porte pas de négatifs d'autres lamelles sur le côté opposé à la facette.

\section{PRODUCTION LAMELLAIRE DES NUCLÉUS PRISMATIQUES}

La production de lamelles de type $\mathrm{D}$ est relativement faible, de 7,66\% (soit 16 lamelles) dans le niveau 11 à un maximum de $17,48 \%$ (soit 25 lamelles) dans le niveau 13.

C'est cependant dans les niveaux les plus anciens (14 et 13) que cette production est la plus importante (en rappelant les réserves concernant la fiabilité des résultats sur les niveaux 13 et 14).

Ces lamelles sont toujours beaucoup plus fragmentées que celles issues des pièces carénées: 89,52 \% des lamelles de l'Aurignacien ancien sont fracturées; dans le niveau 12, il n'y en a même aucune entière. De plus, la majorité des fragments $(47,87 \%)$ sont des fragments mésiaux, ce qui indique une fracturation multiple très importante. Ceci est dû à la plus grande longueur de ce type de lamelles, qui les rend beaucoup plus sujettes à la fracturation, en particulier à la fracturation post-dépositionnelle (fig. 11, $\mathrm{n}^{\circ} 3-6$ ).

Lorsque ce caractère est déterminable, les pièces ne sont presque jamais déjetées; seule une lamelle de la couche 11 est déjetée vers la droite. Ce sont généralement de grandes lamelles: la plupart sont comprises entre $8 \mathrm{~mm}$ et $12 \mathrm{~mm}$ de largeur (fig. 12). Contrairement à ce que l'on pourrait penser, les lamelles issues des nucléus ne sont pas toutes rectilignes. Certes les lamelles rectilignes sont majoritaires $(67,29 \%)$, mais nous avons également une quantité non négligeable de lamelles courbes $(16,82 \%)$, ainsi que quelques lamelles torses $(6,54 \%)$ et quelques lamelles courbes et torses $(5,61 \%)$. La présence de ces différents types de lamelles n'est cependant pas surprenante. En effet, bien que ce facteur n'ait pas été détaillé lors de notre étude globale du matériel de ces niveaux (Chiotti, 1999), nous avons pu constater que ces différents types sont présents dans la production de lames, qui est également issue essentiellement de nucléus prismatiques.

Comme nous l'avons vu dans la présentation des types de lamelles (cf. p. 117), trois schémas différents sont possibles pour expliquer la production des lamelles de type D : à partir de nucléus prismatiques spécifiques, lors de la réduction de nucléus à lames, ou bien des lamelles intercalées dans une production laminaire. Lorsque seules les lamelles sont présentes, il est très difficile, voire impossible, de distinguer de quel schéma de production elles sont issues.

Les nucléus prismatiques à lamelles siricto sensu sont quasiment absents de ces niveaux. Un seul exemplaire a été retrouvé dans le niveau 12 (fig. $11, n^{\circ} 1$ ). Il s'agit d'un petit nucléus ( $45 \mathrm{~mm}$ x $41 \mathrm{~mm}$ x $28 \mathrm{~mm}$ ) dont la face arrière et le fond sont totalement corticaux. Ia position du cortex sur le fond du nucléus et à l'arrière du plan de frappe témoigne des faibles dimensions du bloc d'origine, et atteste donc qu'il s'agit bien, dès le début du débitage, d'un nucléus à intention lamellaire et non d'une réduction d'un nucléus à lames.

Le niveau 12 a également livré un flanc de nucléus portant des négatifs lamellaires (fig. $11, n^{\circ} 2$ ), qui peut 

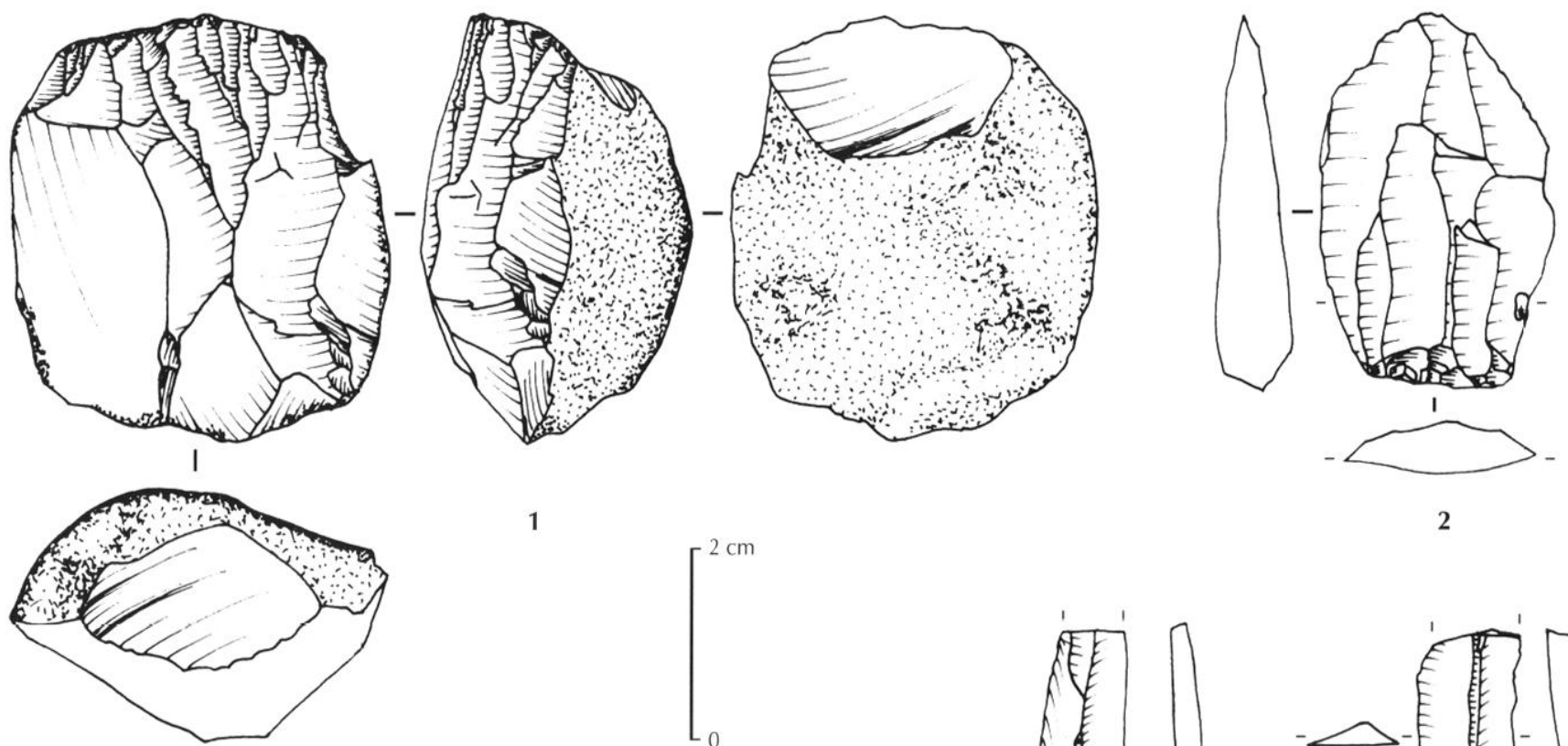

1

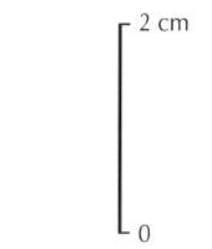

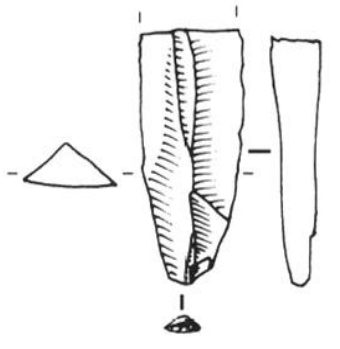

3

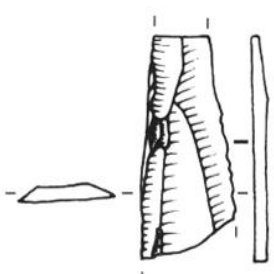

4

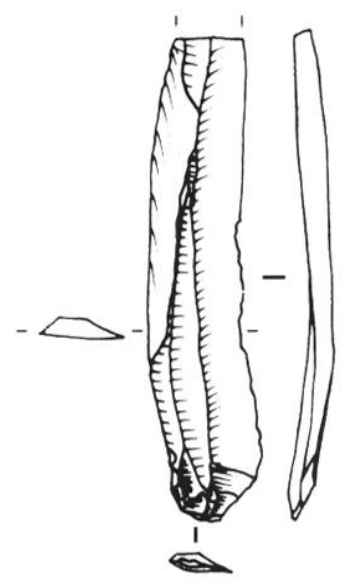

5
2

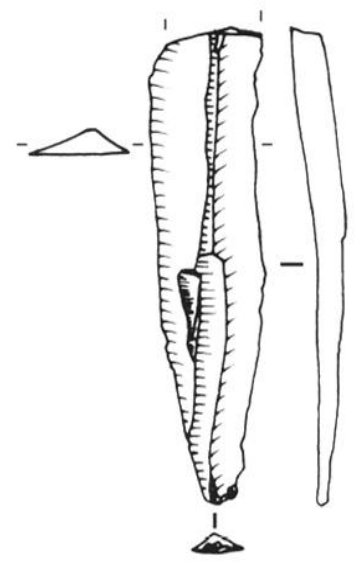

6

Fig. 11 - Production des nucléus prismatiques de l'Aurignacien ancien: 1, nucléus à lamelles du niveau 12 ; 2, flanc de nucléus à lamelles du niveau $12 ; 3,5$, lamelles de type I) du niveau $11 ; 4,6$, lamelles de type 1 ) du niveau 14 (dessins $L$. Chiotti).

lamelles des nucléus prismatiques (type D)

nombre de pièces

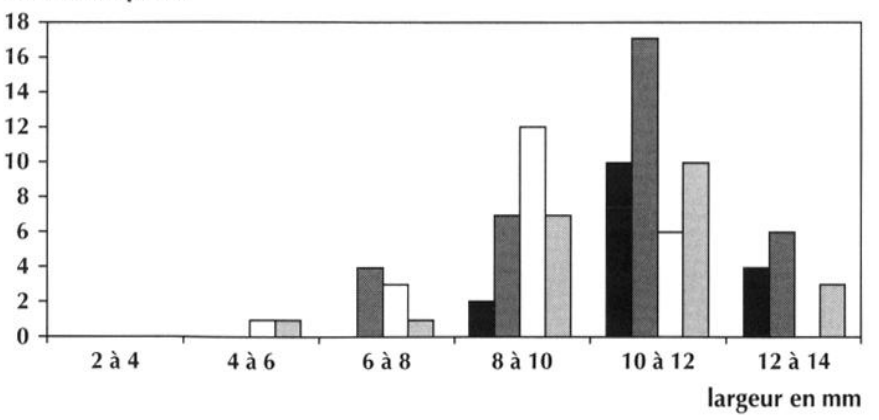

niveau $11 \quad \square$ niveau $12 \quad \square$ niveau $13 \quad \square$ niveau 14

Fig. 12 - Histogramme présentant les largeurs des lamelles provenant des nucléus prismatiques (type D) dans les niveaux d'Aurignacien ancien (niveaux 11, 12, 13 et 14). provenir d'un vrai nucléus à lamelles ou d'un nucléus à lames réduit. Mis à part cela, les quatre niveaux principaux de l'Aurignacien ancien de l'abri Pataud n'ont pas livré d'exemple identifiable de production de lamelles sur des nucléus résultant de la réduction de nucléus laminaires.

En revanche, nous avons pu mettre en évidence une production de lamelles intercalées dans un débitage laminaire grâce à l'exemple fourni par un remontage effectué dans la couche 14 .

Ce remontage concerne un débitage laminaire effectué sur un bloc de silex sénonien noir (fig. 13) dont seulement une petite partie a pu être remontée (probablement du fait du nombre important de pièces qui n'ont pas été retrouvées pour cette couche). Ce remontage est constitué de deux parties séparées par une 


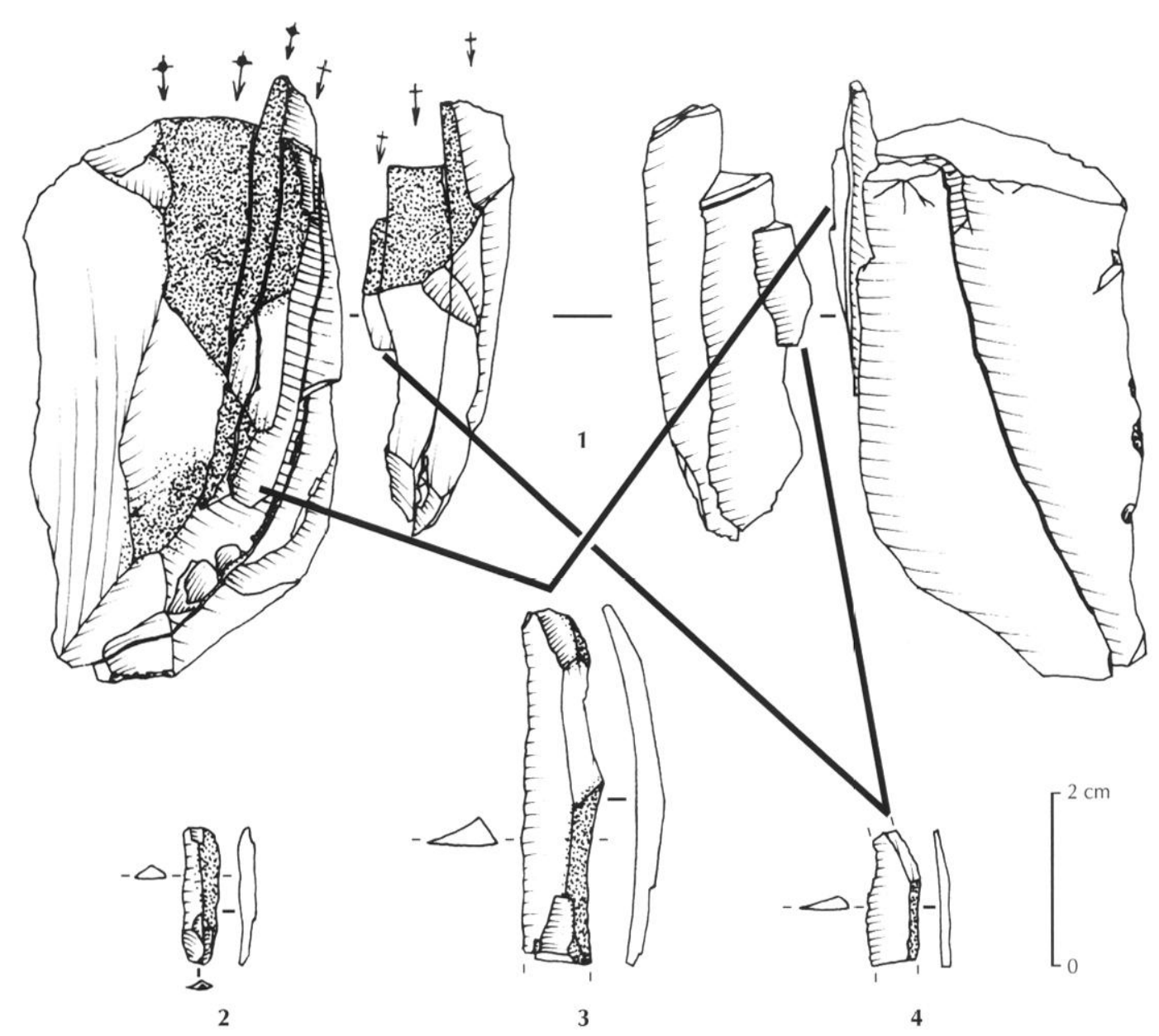

Fig. 13 - Remontage d'une partie de la séquence d'un débitage laminaire incluant des lamelles, provenant du niveau 14 : 1 , remontage ; 2, lamelle non remontée; 3, 4, lamelles remontées, intercalées entre les lames (dessins $L$. Chiotti).

ou deux pièces manquantes (fig. $13, n^{\circ} 1$ ). Deux lamelles sont intercalées entre les différentes lames, et nous avons également une troisième lamelle qui provient de la même séquence mais qui n'a pas pu être remontée. Les deux pièces remontées ne sont pas entières mais leur état de fracturation laisse encore entrevoir qu'elles avaient des gabarits assez différents (fig. 13, $\mathrm{n}^{\circ \mathrm{s}} 2-4$ ). L'une est de grandes dimensions ( $41 \mathrm{~mm} \times 11 \mathrm{~mm} \times 4 \mathrm{~mm}$ ), alors que la seconde est plus petite $(16 \mathrm{~m} \times 8 \mathrm{~mm} \times 2 \mathrm{~mm})$. La lamelle non remontée est encore plus petite $(16 \mathrm{~mm}$ x $5 \mathrm{~mm} \times 2 \mathrm{~mm}$ ), d'autant plus qu'elle est entière. Dans les trois cas il s'agit de lamelles qui présentent un bord cortical, donc produites sur le bord de la surface de débitage. Elles se situent par conséquent dans des phases d'extension latérale de cette surface.

Le problème posé par ces lamelles est de savoir s'il s'agit d'une production volontaire ou opportuniste. Dans l'état actuel de nos travaux, nous n'avons pas vraiment d'arguments à apporter en faveur de l'une ou l'autre de ces hypothèses étant donné le très faible nombre de pièces que nous avons pu rattacher à ce schéma de production. Cependant il nous semble probable qu'il s'agisse en fait de sous-produits et non d'une production volontaire.

En effet, dans la majorité des cas, les lames débitées sur de petits blocs sont de dimensions assez proches de celles des lamelles. Dans ces conditions, les productions laminaires sont assez favorables à une production accidentelle de lamelles, en particulier pour des pièces comme la plus grosse des lamelles figurant dans le remontage. Il ne s'agirait donc pas d'une production volontaire, mais plutôt de quelques lames plus petites que les autres. 


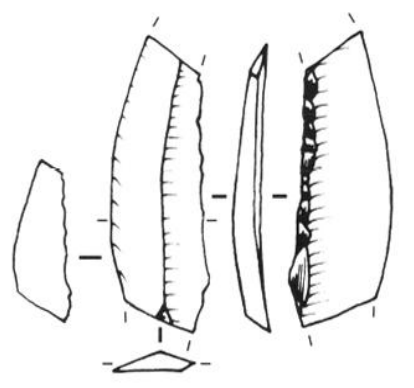

1

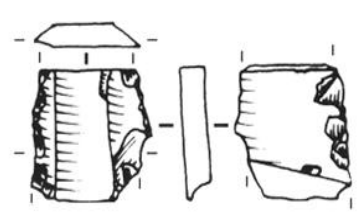

3

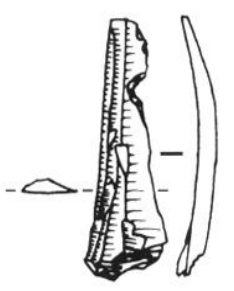

5
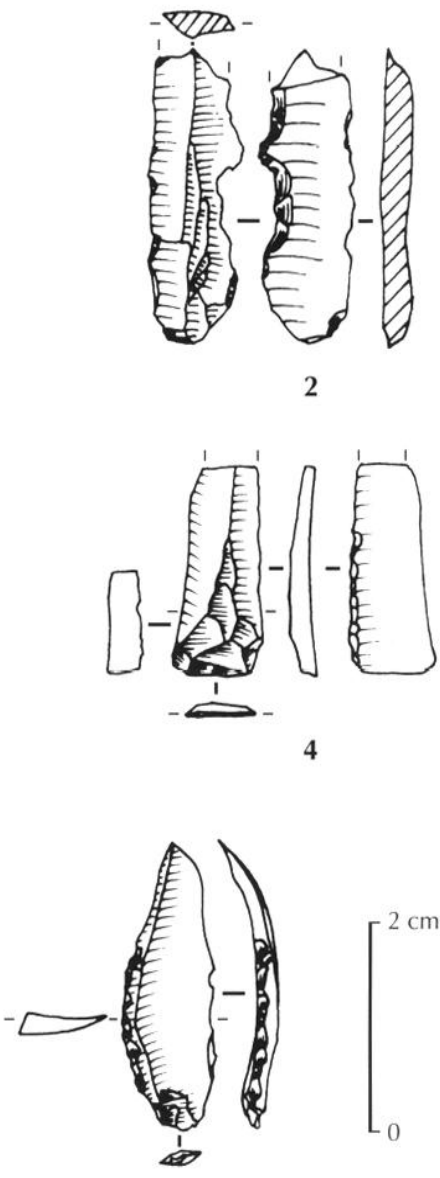

Fig. 14 - Outillage lamellaire de l'Aurignacien ancien: 1, lamelle Dufour du niveau $12 ; 2$, lamelle denticulée du nivean 12 ;

3, lamelle relouchée du niveau $11 ; 4$, lamelle Dufour du niveau 14 ;

5, lamelle à encoche du niveau 12 ; 6, lamelle retouchée du niveau 12 (dessins L. (hiotti).

\section{LAMELLES OPPORTUNISTES}

Dans les quatre niveaux considérés, ces pièces sont peu nombreuses: quinze lamelles dans le niveau 14, treize dans le niveau 13 , quinze dans le niveau 12 et vingttrois dans le niveau 11.

Elles sont assez irrégulières, et ne présentent aucun signe d'une quelconque standardisation, si ce n'est des dimensions similaires pour celles du niveau 11 , mais cela ne semble pas être volontaire. En effet, il s'agit de pièces très différentes les unes des autres qui ont été obtenues accidentellement au cours du développement de diverses chaînes opératoires qui, pour la plupart, n'étaient probablement pas destinées à la production de lamelles.

\section{OUTILLAGE LAMELLAIRE}

Les lamelles transformées en outils sont assez peu nombreuses dans l'Aurignacien ancien, avec seulement 31 pièces. Elles sont réparties très inégalement entre les différents niveaux, puisqu'à lui seul le niveau 12 comprend vingt outils sur lamelles. Il y en a également sept dans le niveau 11 et deux dans les niveaux 13 et 14 .

L'outillage lamellaire de l'Aurignacien ancien est composé de 13 lamelles plus ou moins bien retouchées, 10 lamelles à encoche, 3 lamelles Dufour, 3 lamelles tronquées et 2 lamelles denticulées (fig. 14).

Les supports de ces outils sont constitués par des lamelles d'origines et de formes très diverses:

- lamelles retouchées : 6 lamelles de type D, 4 lamelles indéterminées et 3 lamelles de type B ;

- lamelles à encoche : 4 lamelles de type B, 2 lamelles de type A, 2 lamelles de type $D$ et 2 lamelles indéterminées ; - lamelles Dufour : 3 lamelles de type A ;

- lamelles tronquées : 2 lamelles de type A et 1 lamelle indéterminée ;

- lamelles denticulées : 1 lamelle de type D et 1 lamelle indéterminée.

Les seules standardisations que l'on peut noter concernent les lamelles Dufour qui sont toutes sur supports identiques et les lamelles retouchées qui, bien qu'étant sur des supports de natures différentes, sont toutes de dimensions relativement importantes.

Mis à part ces deux types de pièces, le choix des lamelles utilisées dénote un certain opportunisme, parmi un fonds de lamelles déjà débitées, et non un débitage dans le but d'obtenir des supports particuliers pour les outils.

\section{NIVEAU 8, AURIGNACIEN ÉVOLUÉ}

Les lamelles du niveau 8 faisant l'objet d'une autre étude plus particulière (Chiotti, 2000, à paraître), nous ne présenterons pas ici leur étude détaillée comme pour les autres niveaux. Cependant, afin de rester cohérent et d'obtenir des points de comparaison avec les autres niveaux, nous présenterons un résumé des différentes observations déjà réalisées sur cette industrie.

Tout d'abord, il convient de rappeler que l'industrie du niveau 8 est celle qui a livré le plus de lamelles, avec un total de 1149 pièces. La caractéristique principale de 
ce niveau, concernant l'étude de la production lamellaire, est la quantité de petites lamelles Dufour du soustype Roc de Combe (Demars, Laurent, 1989) présentes dans l'outillage. C'est de loin le niveau le plus riche de l'Aurignacien de l'abri Pataud puisque, avec 46 pièces, les lamelles Dufour représentent $8,38 \%$ de l'outillage. C'est également cette industrie qui a livré la plus grande quantité de grattoirs aurignaciens (carénés et à museau) qui, avec 116 pièces, représentent $20,77 \%$ de l'outillage.

La présence en grande quantité des grattoirs aurignaciens et des lamelles Dufour nous a permis de mener une étude technologique complète basée sur un ensemble de méthodes complémentaires (étude morphologique et morphométrique, remontage, expérimentation). Cette étude (Chiotti, 2000) a pu démontrer de façon certaine le lien technologique unissant ces deux types d'outils, à savoir que les grattoirs aurignaciens sont les nucléus ayant permis d'obtenir les supports des lamelles Dufour (du sous-type Roc de Combe).

\section{PRODUCTION LAMELLAIRE DES GRATTOIRS CARÉNÉS ET À MUSEAU}

Comme celles de l'Aurignacien ancien, les lamelles issues des grattoirs aurignaciens du niveau 8 ont été séparées en deux groupes: des lamelles de type B issues des grattoirs carénés et des lamelles de type A qui peuvent provenir soit de grattoirs carénés, soit des grattoirs à museau épais. Ces dernières sont les plus nombreuses avec 738 pièces, alors qu'il n'y a que 171 lamelles de type B.

Les deux types de grattoirs (carénés et à museau) étant en quantités assez proches (respectivement 55 et 61 pièces), la différence du nombre de lamelles provient du type même de production puisque seule une partie des lamelles des grattoirs carénés est identique à celles des grattoirs à museau. Malgré cela, la différence de quantité est tellement importante qu'il semble que les grattoirs à museau aient produit des quantités plus importantes de lamelles que les grattoirs carénés. Le remontage de l'un des grattoirs à museau (Chiotti, 2000) atteste que ce type de grattoir pouvait fournir un grand nombre de lamelles puisque nous avons pu en identifier 45 provenant de cette seule pièce.

Les lamelles de type B provenant des grattoirs carénés ne sont pas standardisées, quels que soient les critères considérés (morphométrie, courbure, torsion, déviation, etc.). Techniquement, ce sont les premières lamelles détachées durant les phases de mise en forme ou de réaménagement de la table de débitage. Elles se situent, dans la majorité des cas, sur les parties latérales du front. Il s'agit donc de sous-produits issus des phases de préparation des fronts de grattoirs. Ces lamelles sont en fait destinées à permettre le débitage des petites lamelles de type A sur la partie centrale des fronts de grattoirs dont l'épaisseur est trop importante pour les obtenir directement.

Les dimensions des lamelles de type A présentent une très faible dispersion, révélant ainsi un gabarit très standardisé. Elles sont généralement à la fois courbes et torses. En ce qui concerne la déviation, la majorité d'entre elles ne sont pas déjetées, en revanche, les lamelles déjetées sont fortement latéralisées puisqu'elles le sont quasiment toutes vers la droite. Les lamelles courbes et torses déjetées à droite correspondent au gabarit utilisé comme support des lamelles Dufour (Chiotti, id.). Il semble donc que ce soit le gabarit recherché lors de cette production, d'autant plus qu'à l'intérieur même de ces lamelles, il y a eu une sélection importante des plus régulières d'entre elles pour réaliser ces outils.

Le remontage d'une lamelle Dufour dans la séquence de débitage de l'un des grattoirs à museau de cette couche (Chiotti, $i d$.) nous a permis de prouver, pour la première fois de manière directe, que ces grattoirs sont bien les nucléus ayant permis d'obtenir les petites lamelles courbes et torses, déjetées à droite, qui ont servi de supports aux lamelles Dufour. En effet, les remontages de lamelles sur les fronts de grattoirs aurignaciens se sont multipliés ces dernières années (Hahn, Owen, 1984; Le Brun-Ricalens, 1996 ; Marks, Almeida, 1996 ; Zilhão, 1997), mais c'est la première fois qu'un tel remontage inclut une lamelle Dufour. Ce remontage nous a également permis de déterminer en détail la chaîne opératoire permettant le débitage de lamelles sur ces grattoirs, avec l'entretien d'une table de débitage (museau du grattoir) produisant des déchets spécifiques, la production d'une quantité importante de lamelles, mais également beaucoup de petites esquilles et de débris.

\section{PRODUCTION LAMELLAIRE DES BURINS AURIGNACIENS}

Dans le niveau 8 de l'abri Pataud, nous n'avons retrouvé qu'un seul vrai burin caréné pouvant corres- 
pondre à un nucléus à lamelles. Les autres pièces signalées par A. S. Brooks (1979), au nombre de 6, ne sont pas caractéristiques et ont été classées dans d'autres catégories de burins (Chiotti, 1999). De plus, ètant donné la forme de leur support, elles ne peuvent pas avoir servi de nucléus à lamelles.

Les lamelles de type $\mathrm{C}$, caractéristiques d'une production à partir de burins, sont très peu nombreuses dans l'industrie du niveau 8. Nous n'en avons identifié que cinq qui sont toutes de petites lamelles courbes et torses, parmi lesquelles deux sont déjetées à droite et trois sont non déjetées. Dans tous les cas, la facette abrupte permettant de les identifier se situe sur le bord droit de la lamelle.

Il apparaît clairement que la chaîne opératoire de production de lamelles à partir des burins aurignaciens est quasiment inexistante dans l'industrie du niveau 8. Nous pouvons conclure cela malgré le fait que les burins peuvent produire des lamelles identiques à celles des grattoirs à museau. Étant donné le rapport de 1 burin pour 116 grattoirs, nous pouvons donc considérer que les lamelles utilisées comme supports des lamelles Dufour proviennent exclusivement de grattoirs aurignaciens.

\section{PRODUCTION LAMELLAIRE DES NUCLÉUS PRISMATIQUES}

Les pièces provenant de nucléus (lamelles de type D) sont peu nombreuses dans cette industrie, avec seulement 30 pièces, soit $2,61 \%$ des lamelles. Il s'agit en majorité de lamelles rectilignes et non déjetées. Elles sont très fragmentées (une seule pièce entière).

Un seul nucléus ayant produit des lamelles a été retrouvé, mais il s'agit d'une pièce qui, par la suite, a été réutilisée comme support d'un grattoir caréné. Du fait de ce second usage, il est impossible de dire s'il s'agissait d'un vrai nucléus à lamelles ou d'un nucléus laminaire réduit. La plupart de ces lamelles semblent en fait être des pièces intercalées dans des schémas de production laminaire, plutôt que des lamelles issues de nucléus lamellaires. L'existence de lamelles intercalées dans la production laminaire a pu, comme dans le niveau 14 , être prouvée par la réalisation d'un remontage.

Le remontage en question est celui d'un petit galet de silex dans lequel a été développé un débitage laminaire avec très peu de préparation. Il comprend 4 lamelles remontées, ainsi que 10 autres que nous n'avons pas pu remonter. Les lamelles issues de ce galet à débitage laminaire se situent toutes lors des préparations ou des tentatives de reprise du débitage. Elles ne sont jamais présentes lors du plein débitage des lames, même s'il faut reconnaître que le plein débitage est relativement court pour un galet tel que celui-ci.

Ces lamelles sont de formes très variées, si bien qu'une partie aurait certainement été classée dans d'autres catégories sans la réalisation du remontage, et en particulier dans les lamelles opportunistes. Cette variété de formes met en évidence l'existence possible de certains mélanges lors de notre classement. Il est en particulier probable qu'une part (peut-être importante) des 31 lamelles classées comme opportunistes puisse provenir de débitages laminaires tels que celui-ci.

Ces lamelles ne semblent pas faire partie des pièces recherchées lors de cette chaîne opératoire, mais plutôt en être un sous-produit. Elles peuvent donc être considérées comme opportunistes.

\section{LAMELLES OPPORTUNISTES}

Il s'agit de pièces de forme assez irrégulière qui ne semblent pas réellement issues d'une chaîne opératoire bien organisée, destinée à la production de lamelles. Elles sont peu nombreuses, avec 31 pièces, soit 2,7\% des lamelles. Ce sont des lamelles qui ne présentent pas de caractéristiques particulières au niveau de leurs dimensions, elles sont essentiellement rectilignes et ne sont jamais déjetées. Il s'agit d'un groupe de pièces sans aucune homogénéité.

Comme nous l'avons vu ci-dessus, il est probable qu'au moins une partie d'entre elles provient des phases de préparation de certains nucléus laminaires.

\section{OUTILLAGE LAMELLAIRE}

L'outillage sur lamelles du niveau 8 est constitué essentiellement de lamelles Dufour du sous-type Roc de Combe (Demars, Laurent, 1989) : 46 pièces, dont 2 non retrouvées lors de l'étude (Chiotti, 2000). Elles portent toujours une retouche (presque toujours inverse: 39 pièces) sur leur bord droit, le bord gauche n'étant généralement pas retouché. Comme nous l'avons vu, 
Longueur en $\mathrm{mm}$

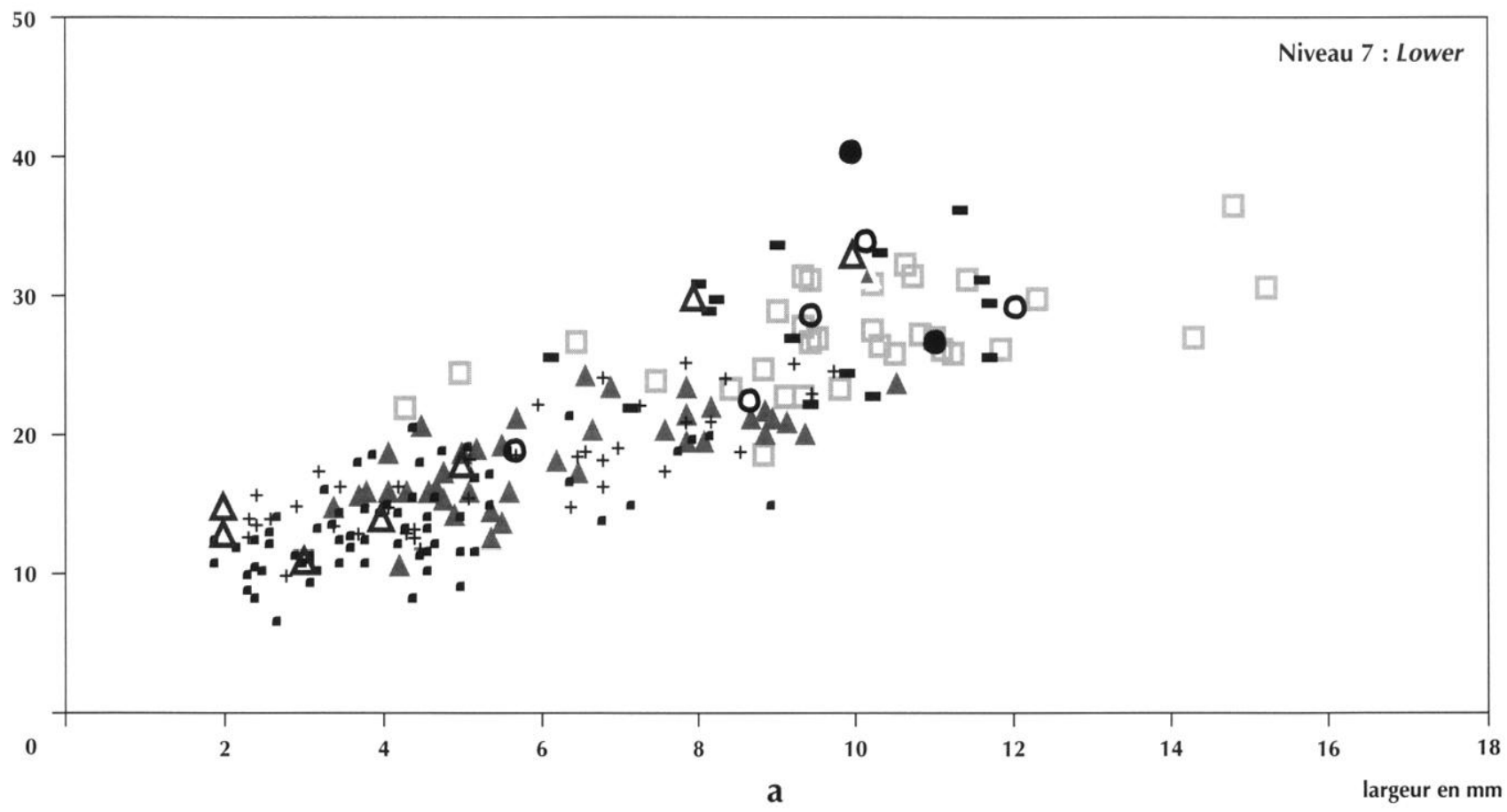

Longueur en $\mathrm{mm}$

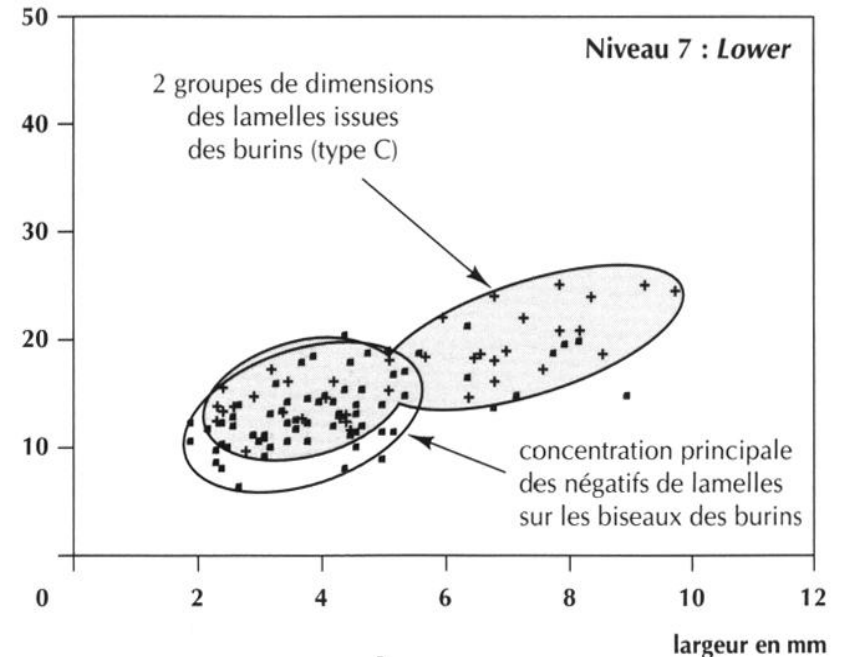

b
Longueur en mm

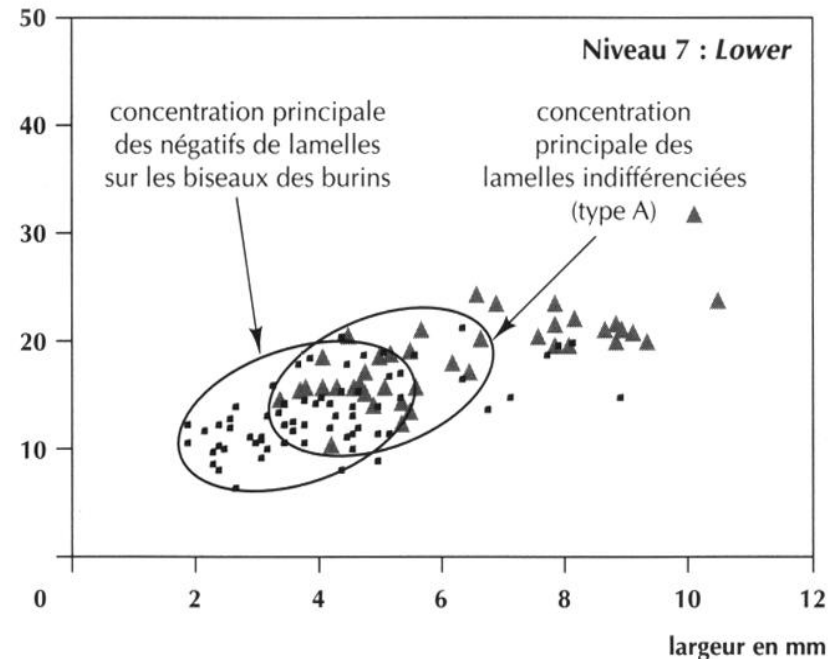

C
ㅁ lamelles des grattoirs carénés (type B)

\ lamelles indifférenciées (grattoirs et burins, type A)

- lamelles indéterminées

- lamelles opportunistes
- lamelles des nucléus (type D)

+ lamelles des burins aurignaciens (type C)

- négatifs de lamelles sur les biseaux des burins

$\Delta$ négatifs de lamelles sur les fronts des grattoirs

Fig. 15 - Diagrammes présentant les rapports longueur/largeur des différents types de lamelles entières dans le niveau $7:$ Lower.

$a$ : toutes les lamelles; $b$ : comparaison des lamelles des burins (type $C$ ) et des négatifs sur les biseaux de burins; $c$ : comparaison des lamelles indifférenciées (type $A$ ) et des négatifs sur les biseaux de burins. 
elles sont réalisées sur les lamelles de type A produites sur les grattoirs carénés ou les grattoirs à museau épais, qui constituent l'essentiel de la production lamellaire du niveau 8 , et plus particulièrement sur celles qui sont courbes et torses, et déjetées à droite.

Les lamelles Dufour ne sont cependant pas les seuls outils sur lamelles du niveau 8 . Nous avons dénombré 17 autres outils constitués de 8 lamelles retouchées (de façon plus ou moins intense), 5 lamelles tronquées, 2 lamelles à encoche, 1 lamelle denticulée et 1 lamelle à dos. Ces outils sont réalisés indifféremment sur tous les types de lamelles. Les supports semblent avoir été choisis au hasard, parmi un ensemble de pièces disponibles. Il n'y a aucun type d'outil qui corresponde à l'un des types de lamelles plutôt qu'à un autre.

Dans la majorité des cas, il s'agit d'outils aménagés seulement par quelques retouches, de facture assez fruste. La pièce la plus transformée est la lamelle à dos.

\section{NIVEAU $7:$ LOWER, AURIGNACIEN ÉVOLUÉ}

Le niveau $7:$ Lower est, avec 332 pièces, le plus riche en lamelles après le niveau 8 (tabl. I). Ce matériel est composé d'une majorité de lamelles de type A (111 pièces, soit 33,43\%) dont nous n'avons pas pu distinguer la provenance, entre les grattoirs carénés, les grattoirs à museau et les burins aurignaciens (busqués, carénés et des Vachons). Mis à part ces lamelles indifférenciées, le niveau 7 : Lower a livré 85 lamelles de type B issues de grattoirs carénés $(25,60 \%), 49$ lamelles de type C issues de burins aurignaciens $(14,76 \%), 31$ lamelles de type $\mathrm{D}$ issues de nucléus prismatiques $(9,34 \%), 18$ lamelles opportunistes $(5,42 \%)$ et 38 lamelles indéterminées $(11,45 \%)$.

\section{PRODUCTION LAMELLAIRE DES GRATTOIRS CARÉNÉS ET À MUSEAU}

\section{LES GRATTOIRS-NUCLÉUS}

Comme dans l'Aurignacien ancien, les grattoirs aurignaciens sont assez peu nombreux. Ils ne représentent que 22 pièces, soit $3,17 \%$ de l'outillage du niveau 7 : Lower. Il s'agit de 14 grattoirs carénés (dont 2 doubles et
3 atypiques), 8 grattoirs à museau et autres grattoirs épais. Leurs supports sont constitués de 14 éclats, 7 blocs et 1 lame. Les fronts de ces grattoirs ont des aspects et des dimensions très variables, avec des largeurs comprises entre $12 \mathrm{~mm}$ et $44 \mathrm{~mm}$ et des épaisseurs entre $12 \mathrm{~mm}$ et $33 \mathrm{~mm}$.

Onze grattoirs ont au moins une encoche adjacente au front : cinq ont cette encoche sur le bord gauche, trois l'ont sur le bord droit, et les trois autres en ont une de part et d'autre du front. Parmi ces pièces, cinq sont des grattoirs carénés à coches (Ronen, 1964), présentant des aménagements issus de la partie supérieure des pièces.

Dans cette industrie, nous avons également retrouvé neuf ravivages de grattoirs carénés destinés à l'entretien des encoches, (Chiotti, 2000). Six ont été détachés sur la gauche du front, un sur la droite et un de la partie supérieure.

\section{LES LAMELLES DE TYPE B}

Comme dans les niveaux précédemment étudiés, nous avons isolé des lamelles de type B, provenant uniquement des grattoirs carénés, qui sont assez nombreuses ( 85 pièces).

Leur rapport longueur/largeur présente une dispersion relativement faible, avec une concentration de largeurs comprises entre $8 \mathrm{~mm}$ et $12 \mathrm{~mm}$ et de longueurs comprises entre $25 \mathrm{~mm}$ et $35 \mathrm{~mm}$ (fig. 15a). Même si la dispersion est plus faible que dans les niveaux précédents, il faut noter qu'il existe un nombre non négligeable de lamelles qui s'écartent de ce gabarit dimensionnel. La prise en compte de la largeur de l'ensemble des lamelles (fig. 16) confirme l'existence de ce gabarit, avec un pic entre $7 \mathrm{~mm}$ et $12 \mathrm{~mm}$, centré sur une largeur de $9 \mathrm{~mm}$.

Les lamelles de type B sont en majorité non déjetées $(59,30 \%)$. Parmi celles qui sont déjetées, il n'y a pas de latéralisation très nette $(16,28 \%$ déjetées à droite et $6,98 \%$ déjetées à gauche). Leurs formes sont extrêmement variées, avec $29,41 \%$ de lamelles courbes et torses, $23,53 \%$ de lamelles torses, $21,18 \%$ de lamelles courbes et $15,19 \%$ de lamelles rectilignes. Il semble donc que, malgré ce que pouvait laisser penser leur étude morphométrique, les lamelles de type B ne sont pas plus standardisées que dans les autres niveaux, ce qui, en définitive, paraît normal puisqu'il s'agit, comme dans ces derniers, 


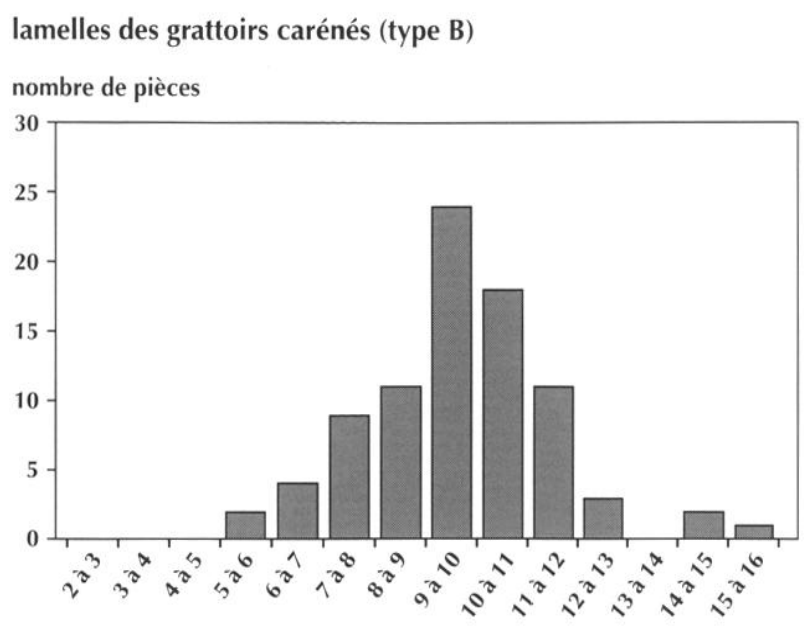

largeur en $\mathrm{mm}$

lamelles indifférenciées (grattoirs et burins, type A)

nombre de pièces

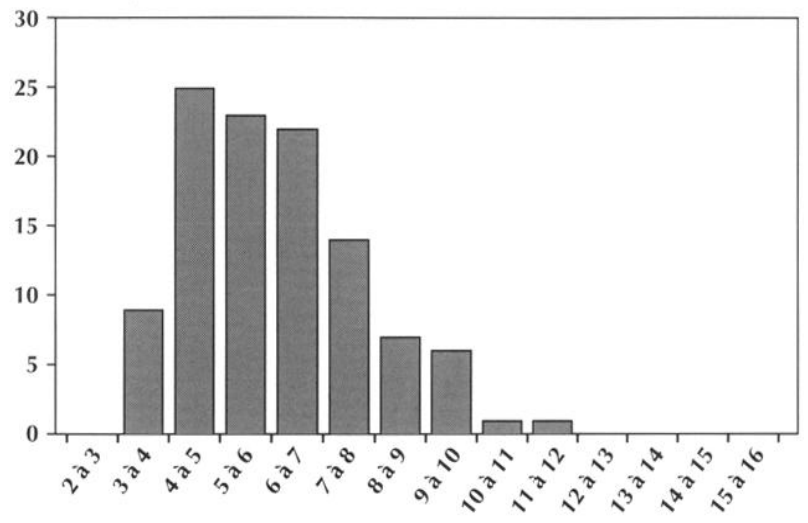

largeur en mm lamelles des burins aurignaciens (type C)

nombre de pièces

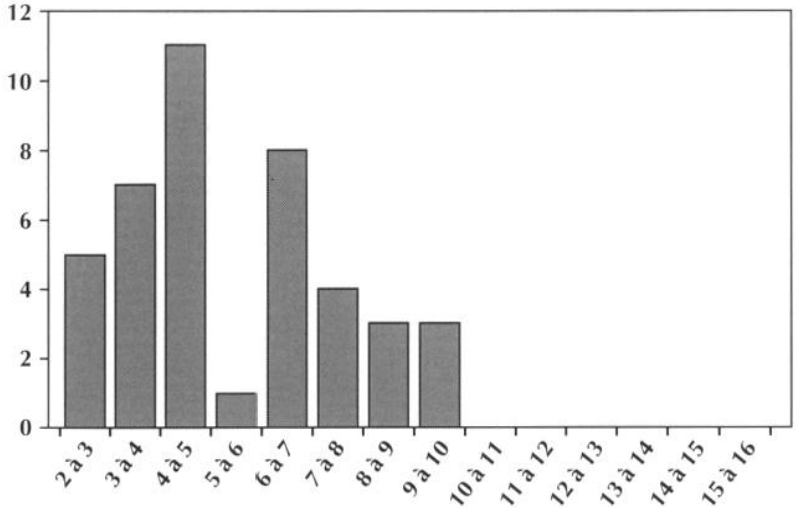

largeur en mm

lamelles des nucléus prismatiques (type D)

nombre de pièces

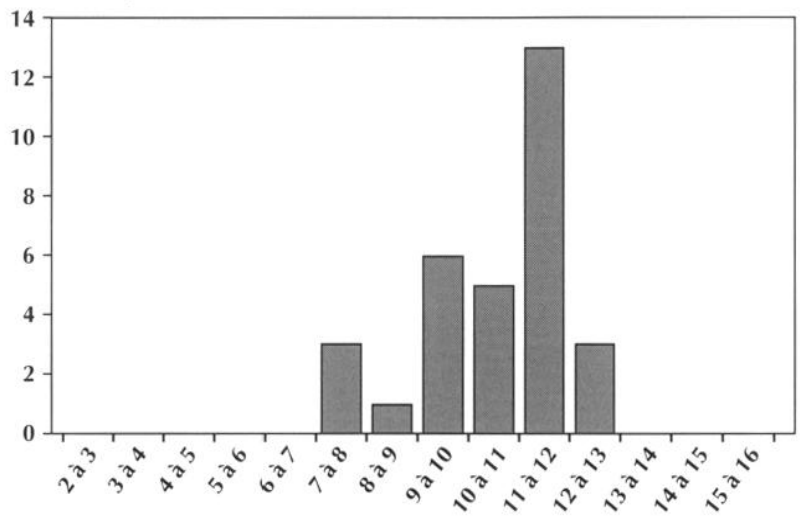

largeur en $\mathrm{mm}$

Fig. 16 - Histogrammes présentant les largeurs des lamelles des grattoirs carénés (type B), des lamelles des burins aurignaciens (type (C), des lamelles indifférenciées (type A) et des lamelles des mucléus prismatiques (type I)) dans le niveau 7 : Lower.

de lamelles destinées à la mise en forme de la table de débitage que constitue le front des grattoirs.

\section{PRODUCTION LAMELLAIRE DES BURINS AURIGNACIENS}

\section{LES BURINS-NUCLÉUS}

Les burins aurignaciens (busqués, carénés et des Vachons) constituent la seconde chaine opératoire importante utilisée par les Aurignaciens pour obtenir des petites lamelles. Malgré l'exemple que nous avons vu précédemment dans le niveau 13, cette technique se rencontre généralement dans l'Aurignacien évolué. À l'abri Pataud, c'est dans le niveau $7:$ Lower qu'elle est la mieux représentée. En effet, ce niveau est celui qui a livré la plus grande quantité de burins aurignaciens, essentiellement des burins busqués (fig. 17 et 18). Les burins aurignaciens simples sont au nombre de 80 dans cette industrie, dont 62 burins busqués, 14 burins des Vachons et 4 burins carénés. En ajoutant 3 burins busqués doubles ( 6 biseaux), 3 burins busqués sur des outils composites et 8 sur des burins multiples mixtes, nous arons dans cette industrie un total de 97 biseaux de burins aurignaciens. Les supports de ces burins sont généralement des lames (51 pièces), mais peuvent aussi être des éclats (32 pièces). 

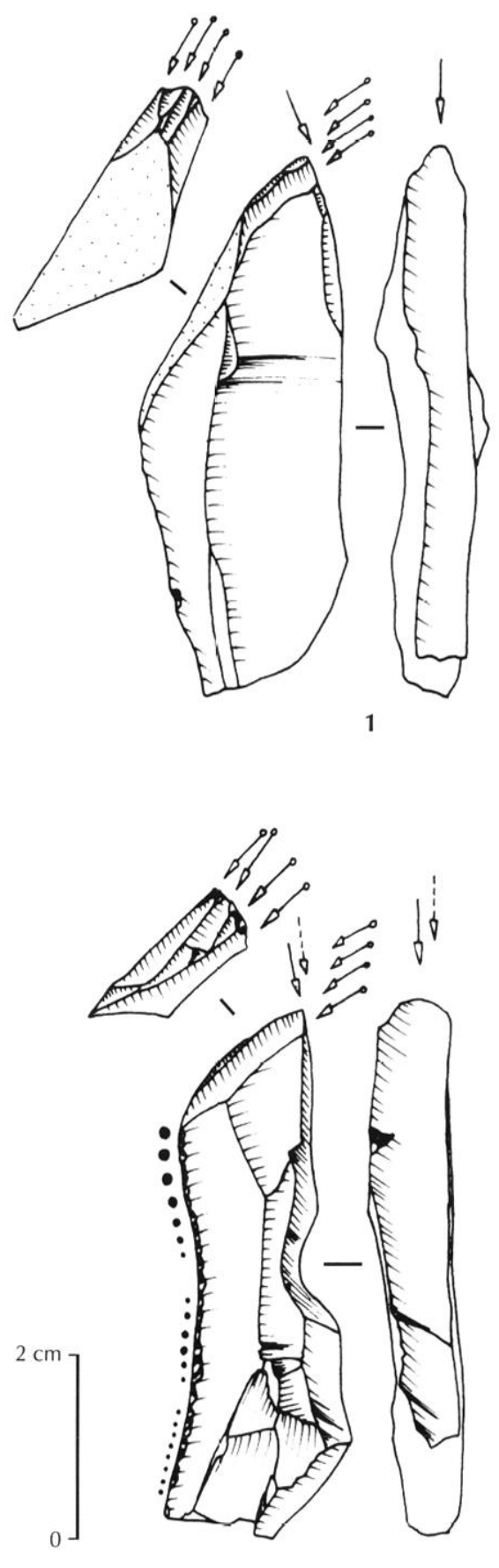

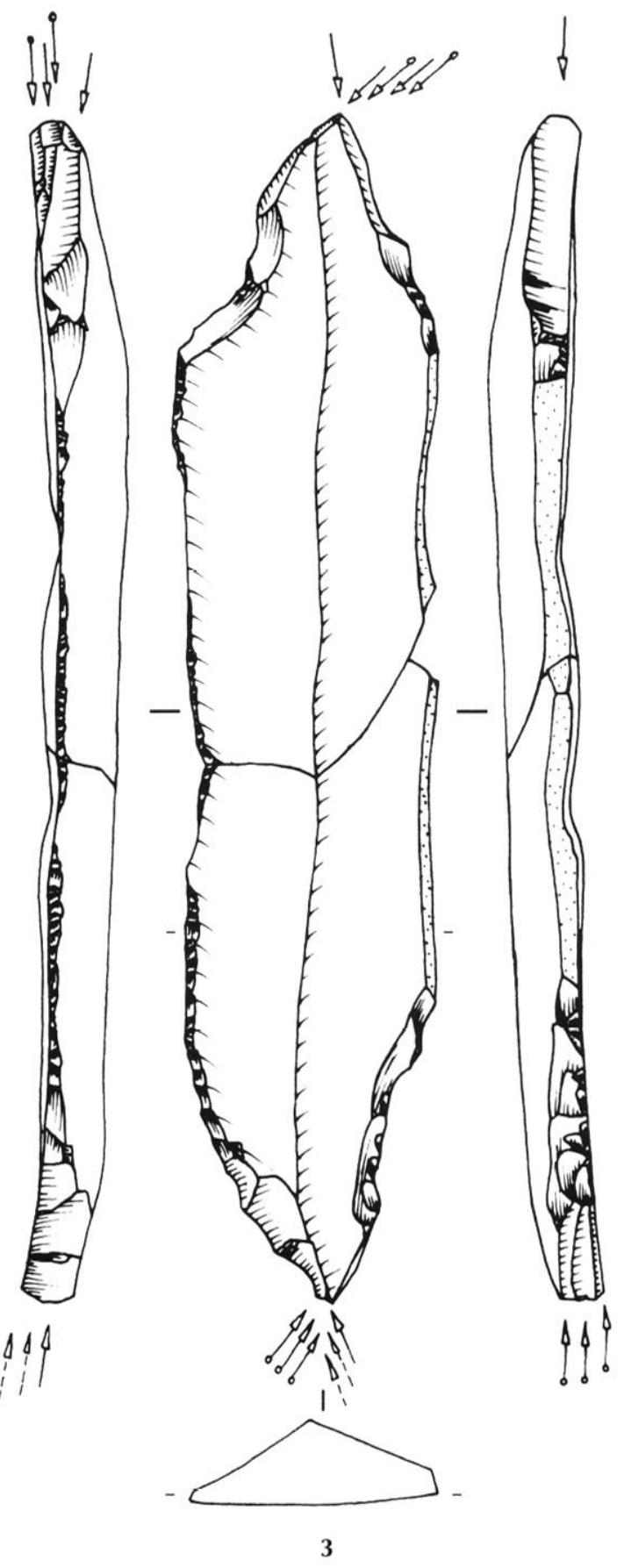

Fig. 17 - Burins aurignaciens du niveau 7 : Lower.
Soixante-cinq biseaux sur quatre-vingt-dix-sept sont réalisés en partie proximale des supports, ce qui est sans doute lié à la recherche de l'épaisseur maximale, c'est-àdire d'une quantité de matière plus importante, mieux adaptée au débitage des lamelles. Il faut d'ailleurs signaler que les supports laminaires des burins aurigna- ciens ont généralement été choisis parmi les lames les plus épaisses produites dans cette industrie.

Sur tous les sites où ils sont présents, les burins aurignaciens sont des pièces très fortement latéralisées (Perpère, 1972a, 1972b ; Demars, 1977, 1982 ; Chiotti, 1999 ; Lucas, 2000). Ceux du niveau 7 : Lower de l'abri 


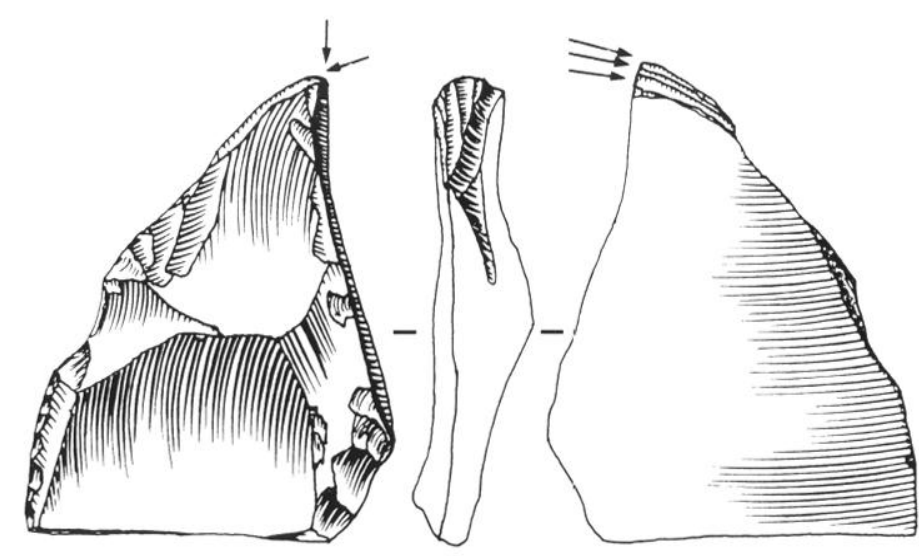

1
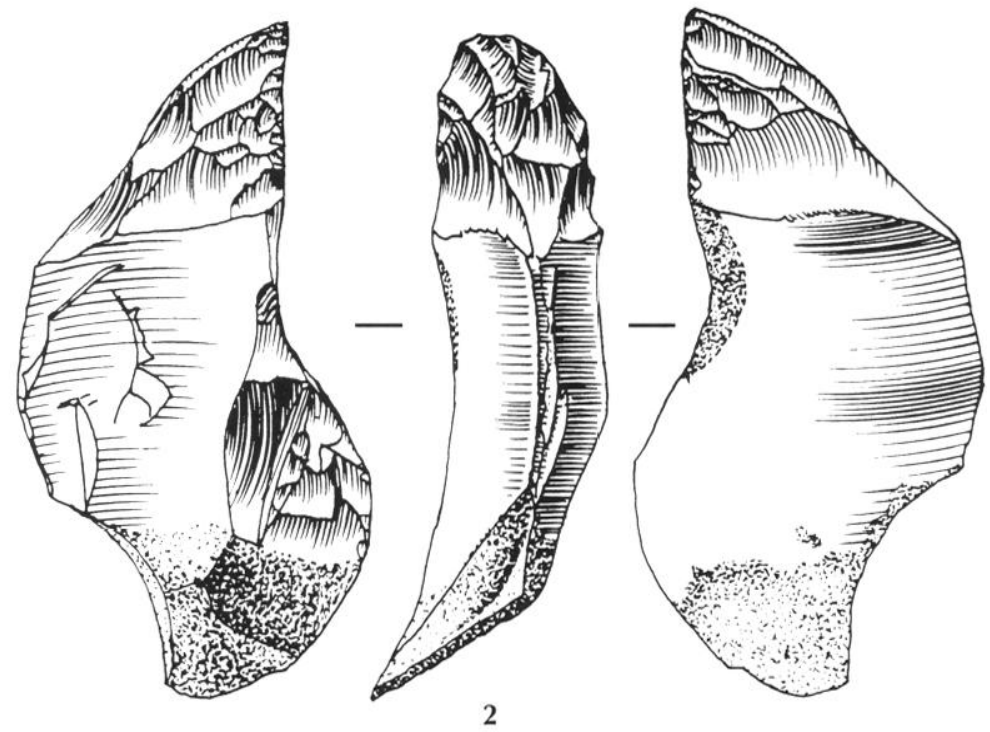

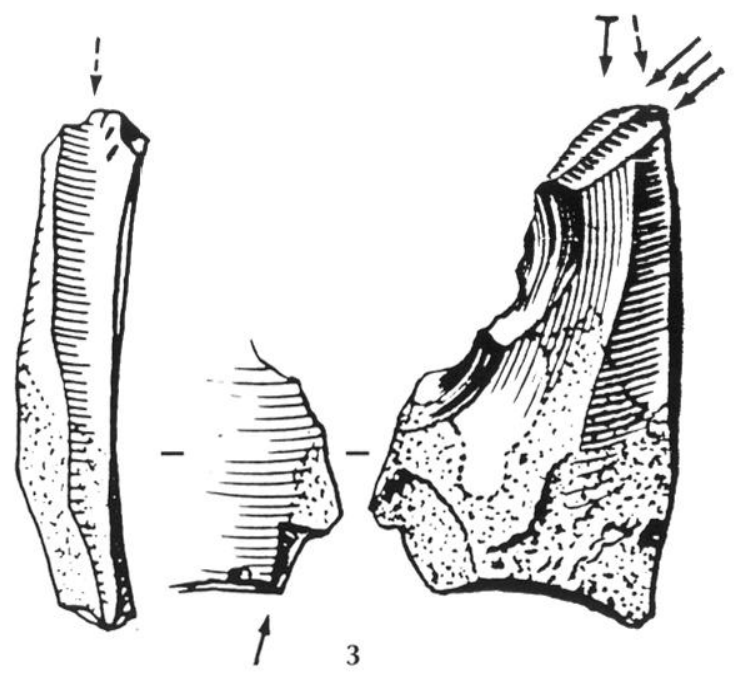

Fig. 18 - Burins aurignaciens du niveau 7 : Lower. 1, 3, burins busqués; 2, burin des Vachons (dessins M. Dauvois).
Pataud ne font pas exception puisque sur 93,81\% d'entre eux, le plan de frappe est sur la droite, et la table de débitage sur la gauche (lorsque la pièce est posée sur sa face d'éclatement, le biseau orienté vers le haut). Selon P.-Y. Demars, cette latéralisation " peut être liée soit à des facilités d'utilisation, soit de fabrication, [mais elle risque d'être liée à la] proportion entre droitiers et gauchers " (Demars, 1982).

Les burins aurignaciens du niveau $7:$ Lower présentent des stigmates d'utilisation qui laissent penser que ces pièces ne sont pas uniquement des nucléus. Il est probable que par suite de leur abandon en tant que nucléus, ils aient été réutilisés, mais cette fois-ci comme outils. Ceci est d'autant plus probable que les stigmates présents sur les burins sont très importants. Il s'agit de deux types de stigmates particuliers : des fractures qui se localisent généralement très près de l'extrémité du biseau, et un poli très important qui généralement ne se situe pas sur le biseau lui-même, mais sur l'un des bords retouchés de la lame servant de support au burin.

Les fractures localisées au niveau de l'encoche d'arrêt des burins busqués, ou juste en arrière, sont fréquentes. Elles concernent 23 burins busqués sur les 65 et peuvent être de deux types.

- Des fractures par flexion (qui n'ont jamais développé de vraie languette) affectent 16 pièces (fig. 19, $n^{\circ} 1-3$ ) ; elles sont presque systématiquement obliques par rapport à l'axe du support, et toujours dans le même sens: la partie la plus longue étant celle qui porte le pan primaire (ancien plan de frappe) et la plus courte celle qui porte le plan secondaire (ancienne table de débitage); elles ne sont pas liées à des dommages 

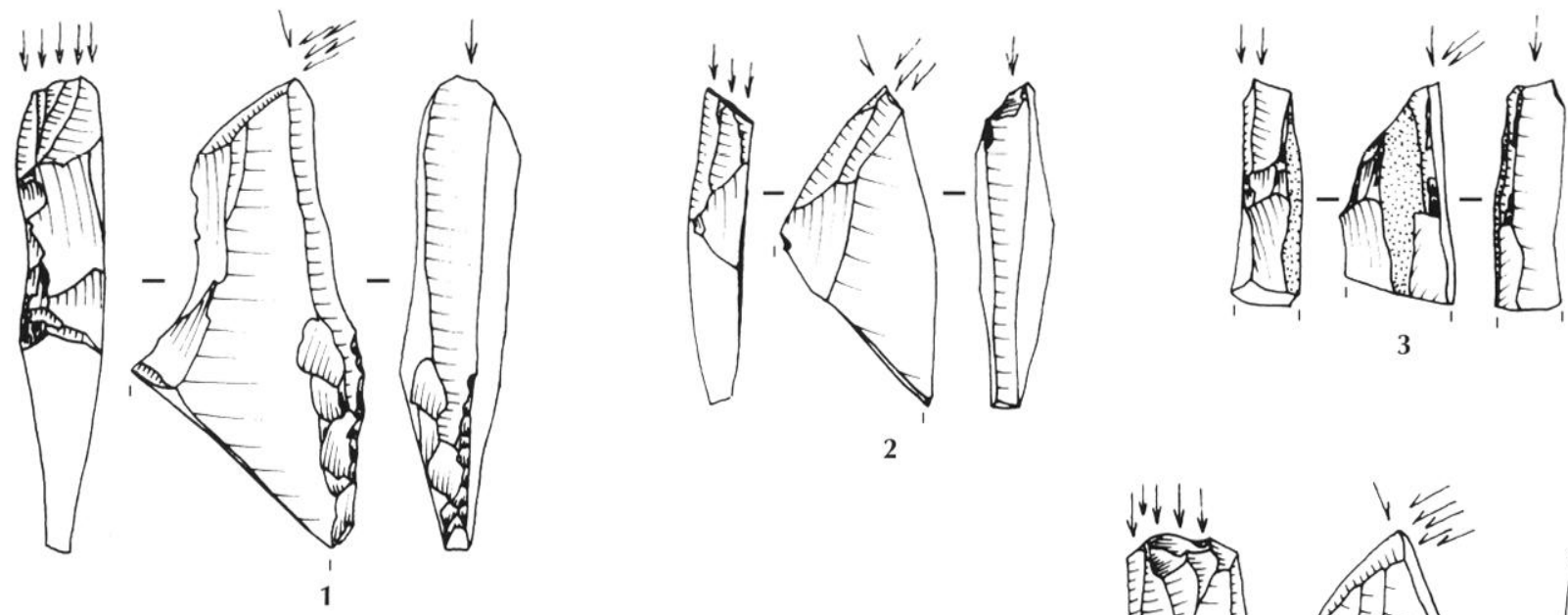

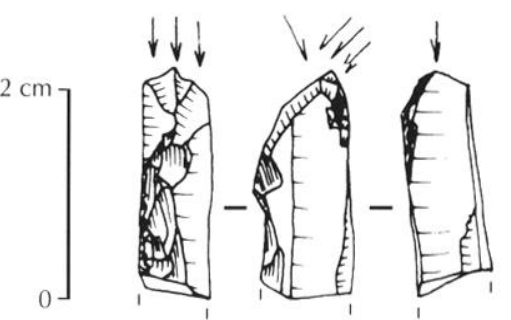

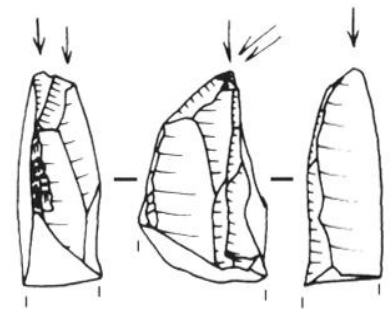

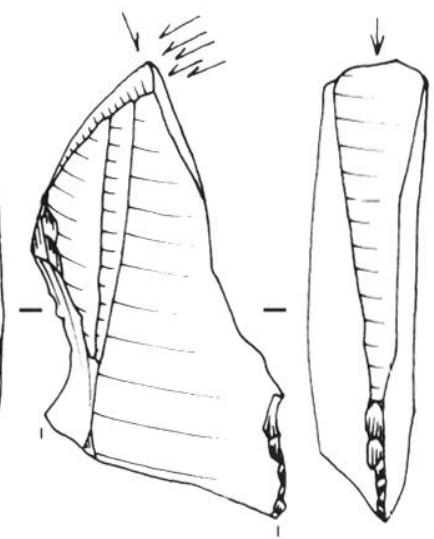

6

Fig. 19 - Burins busqués fracturés du niveau 7 : Lower. 1-3, burins à fracture par flexion; 4-6, burins à fracture transversale (dessins 1. . (.hiotti).

sur le biseau lui-même, ce dernier étant généralement intact.

- Des fractures affectent la pièce dans le sens transversal, mais, à une seule exception près elles n'ont pas développé de languette (fig. 19, n"s 4-6) ; elles affectent 6 pièces pour lesquelles le biseau est toujours intact. Elles semblent résulter d'une pression importante exercée sur le pan primaire du burin. Toutes ces pièces avaient l'extrémité du biseau étroite et allongée. Elles se sont cassées au niveau du point de faiblesse, c'est-à-dire au niveau de l'encoche d'arrêt.

Le poli n'est pas présent que sur des burins aurignaciens. Il affecte également 33 burins de tous types pour lesquels au moins un des bords retouchés est poli. Il s'agit dans la plupart des cas d'un bord portant une retouche marginale courte (fig. 17, $n^{\circ} 2$ ). Il semble bien s'agir de traces liées à l'utilisation des burins, car bien qu'elles soient localisées sur les bords retouchés, elles ne sont présentes que sur les burins, jamais sur les lames retouchées. D'autre part, la présence d'un poli identique sur tous les types de burins semble indiquer que l'usage des burins aurignaciens, après leur rôle de nucléus, était similaire à celui des autres burins.

Ces traces peuvent correspondre à ce que M. Dauvois a appelé abrasion et qu'il a défini comme correspondant « à une perte de matière due à une usure par frottement continu et répété, en va-et-vient ou non " (Dawvois, 1983). L. Pradel parle également de traces d'abrasion, voire même de poli, qui pourraient être obtenues par un travail prolongé sur des corps de dureté moindre (Pradel, 1973).

Nous avons vu qu'au moins une partie des burins aurignaciens avait été utilisée comme outils, mais il semble que certains n'aient été que des outils (du moins pour ce qui concerne leur utilisation sur le site). En effet, lors de notre étude de l'ensemble du matériel aurignacien (Chiotti, 1999), nous avions noté que ce niveau contenait une quantité importante de silex importé de la région de Bergerac, en particulier dans l'outillage $(10,32 \%)$. Les burins aurignaciens présentent également un nombre important de pièces en silex du Bergeracois : 18 sur un total de 83. Or ce n'est absolument pas le cas parmi les 
lamelles. Celles qui sont identifiées comme provenant des burins (type $\mathrm{C}$ ) ne contiennent que six pièces en silex du Bergeracois et celles qui sont indifférenciées (provenant des grattoirs ou des burins, de type A) n'en contiennent que cinq.

Il apparaît donc qu'une partie des burins aurignaciens ont été introduits dans l'abri déjà transformés en burins ${ }^{4}$. Nous avons donc ici un argument supplémentaire de l'utilisation secondaire des burins aurignaciens en tant qu'outils. En effet, dans le cas d'un apport sous leur forme finale, ils n'ont pu être introduits que pour servir d'outils. Cependant, cela ne signifie pas que, dans un premier temps, ils n'ont pas servi de nucléus à lamelles, mais simplement qu'ils n'ont pas eu cette fonction sur le site.

Les tables de débitage (ou pans secondaires des biseaux) de ces burins sont très standardisées, contrairement aux biseaux de tous les autres types de burins de cette industrie (Chiotti, 1999, graph. 120, p. 526), ce qui montre bien qu'ils avaient certainement une fonction différente des autres burins, à savoir celle de nucléus. La plupart de ces tables de débitage ont une largeur comprise entre $5 \mathrm{~mm}$ et $11 \mathrm{~mm}$, pour une longueur qui ne dépasse qu'exceptionnellement $20 \mathrm{~mm}$. Ces tables de débitage ont un gabarit qui correspond à celui des lamelles de type C. En effet, pour la longueur, qui est la seule dimension comparable, ces lamelles sont pour la plupart comprises entre $12 \mathrm{~mm}$ et $20 \mathrm{~mm}$.

Le plan de frappe (ou pan primaire des burins) est généralement formé par un aménagement, de type coups de burins $(82,29 \%)$, mais également parfois par un pan naturel $(17,71 \%)$. L'aménagement ou le ravivage a produit des déchets caractéristiques, déjà décrits par F. Le Brun-Ricalens et L. Brou, sous le nom de " tablette lamellaire de type Thèmes " (Le Brun-Ricalens, Brou, sous presse), et par M. Otte sous le nom d'" enlèvements rectilignes de burins carénés " (Otte, 1979). Ils sont identiques à de grandes chutes de burins, tout à fait classiques ; le seul élément diagnostique entre ces deux types

4. La réalisation d'un remontage dans l'un de ces silex du Bergeracois (Chiotti et al., à paraître) nous a cependant permis de savoir qu'une partie des burins aurignaciens réalisée dans ce matériau, a été produite et débitée dans l'abri, même si très peu de lamelles/chutes de burins y ont été retrouvées. Cela concerne au moins un burin busqué et deux burins busqués doubles (fig. $17, \mathrm{n}^{\circ} 3$ ) qui font partie du remontage. Il faut par ailleurs noter que sur les 6 lamelles de type $C$ en silex du Bergeracois, 5 sont dans la matière du remontage et sur les 5 lamelles de type A (indifférenciées), 3 sont dans cette même matière. de pièces étant la présence, sur le talon, de facettes correspondant aux négatifs des enlèvements lamellaires courbes. Cependant ces dernières ne sont présentes que sur les ravivages de plan de frappe, et pas sur les aménagements de premier ordre. De plus, elles peuvent parfois être confondues avec les restes de la troncature des burins sur troncature.

\section{LES LAMELLES DE TYPE $\mathbf{C}$}

Comme nous l'avons vu précédemment, en particulier dans le niveau 13, nous avons pu identifier une partie des lamelles provenant du débitage des burins aurignaciens (lamelles de type C). Il s'agit des lamelles qui sont détachées sur la partie du biseau jouxtant la face d'éclatement du support, et qui ont emporté une partie de cette face d'éclatement. Cette particularité, qui se traduit par la présence d'une petite facette abrupte sur le bord de la lamelle, permet de les identifier (fig. 20, $n$ 's 2-5).

Ces lamelles sont au nombre de 49 dans l'industrie du niveau 7 : Lower, mais les lamelles ainsi identifiées ne représentent qu'une partie relativement faible des lamelles produites à partir des burins aurignaciens. En effet, étant donné la quantité de ces pièces et les possibilités de production d'un seul de ces burins (Le BrunRicalens, Brou, sous presse; cf. p. 126), un grand nombre de lamelles a dû être produit dans cette industrie. Les possibilités de production peuvent également être illustrées par l'un des burins busqués du niveau $7:$ Lower pour lequel nous avons retrouvé deux des chutes d'aménagement du pan primaire (fig. 20, $n^{\circ} 1$ ). Ces pièces n'ont pas pu être remontées directement, cependant, leur matière très reconnaissable (silex du Bergeracois), la position du cortex, et la forme des chutes et du burin nous permettent de les relier sans risque d'erreur, et même de situer avec une relativement bonne précision la position des chutes par rapport au burin. Le positionnement de ces chutes indique un raccourcissement de la lame servant de support d'au moins $36 \mathrm{~mm}$, ce qui laisse entrevoir qu'un nombre conséquent de lamelles ont pu en être extraites.

La faible quantité de lamelles retrouvées peut avoir deux origines : d'une part, les lamelles détachées sur la partie du biseau se situant vers la face supérieure du support n'ont pas de facette abrupte et sont alors impossibles à distinguer des lamelles produites par les grattoirs aurignaciens ; d'autre part, ces pièces étant de très faibles 
dimensions, il est probable qu'une partie d'entre elles n'ait pas été récoltée lors de la fouille ${ }^{5}$.

L'étude morphométrique de ces lamelles - rapport longueur/largeur (fig. 15a) et histogramme des largeurs (fig. 16) - révèle une séparation en deux groupes de dimensions différentes.

Un premier groupe de petites lamelles (entre $2 \mathrm{~mm}$ et $6 \mathrm{~mm}$ de largeur et entre $12 \mathrm{~mm}$ et $18 \mathrm{~mm}$ de longueur) présente une très faible dispersion et un second groupe de lamelles plus grandes (entre $6 \mathrm{~mm}$ et $10 \mathrm{~mm}$ de largeur et entre $15 \mathrm{~mm}$ et $25 \mathrm{~mm}$ de longueur) est beaucoup plus diffus. Le premier groupe se superpose avec les points représentant le rapport longueur/largeur des négatifs mesurés sur les tables de débitage des burins qui forment eux-mêmes un nuage très groupé (fig. 15b). Cependant quelques points se superposent plus ou moins avec la seconde partie plus diffuse du nuage de points.

Les petites lamelles situées dans la partie basse du nuage constituent en fait le produit principal de la chaîne opératoire des burins busqués. De plus, la superposition des nuages de points représentant les lamelles et les négatifs sur les burins montre qu'une partie de ces derniers sont de dimensions inférieures aux lamelles les plus petites. Ceci nous conforte dans notre hypothèse formulée précédemment selon laquelle toutes les lamelles n'ont pas été récoltées lors de la fouille, et en particulier les plus petites.

Les lamelles du second groupe (les plus grandes) sont souvent des pièces épaisses qui ont emporté une partie importante de la surface de débitage et qui portent un grand nombre de négatifs de lamelles. La plupart d'entre elles ont emporté accidentellement une partie de la table de débitage (fig. $20, \mathrm{n}^{\circ} 7$ ), mais d'autres proviennent du ravivage de cette table de débitage (fig. $20, \mathrm{n}^{\circ} 6$ ).

La grande majorité des lamelles de type $\mathrm{C}$ ( 40 pièces sur 49) sont courbes et torses. Il y en a également 7 courbes et 2 rectilignes. Les lamelles non déjetées dominent avec 29 pièces. Il y a quand même beaucoup de lamelles déjetées à droite ( 17 pièces), en revanche, il n'y a que 3 lamelles déjetées à gauche.

Cette déviation vers la droite correspond à celle observée sur les négatifs présents sur les biseaux des

5. Le tamisage des sédiments n'a pas été homogène durant toute la durée de la fouille. Lors de la fouille du niveau 7 (saison 1961), le tamisage n'a pas été systématique, et lorsqu'il avait lieu, il s'agissait de tamisage à sec (renseignements H. M. Bricker). Ceci est certainement à l'origine de la perte d'une fraction très fine du matériel archéologique. burins aurignaciens. Parmi ces derniers, les pièces déjetées à droite sont même dominantes (41 négatifs sur 72). Les autres négatifs sont essentiellement non déjetés (26), seuls 5 sont déjetés à gauche.

Ces lamelles présentent également une seconde latéralisation : celle de la position de la facette abrupte. La facette se situe essentiellement sur le bord droit des lamelles ( 42 pièces sur 49 ). Cette latéralisation est liée à celle des burins dont elles sont originaires. En effet, si le pan secondaire du biseau est à gauche, la facette sera sur le bord droit de la lamelle et inversement. Comme pour la plupart des burins, le pan secondaire est à gauche, la majorité des chutes ont leur facette à droite.

En conclusion, il apparaît que la chaine opératoire de production de lamelles à partir des burins aurignaciens a fourni des lamelles de très petites dimensions, souvent inférieures à celles des grattoirs, qui sont en très grande majorité courbes et torses, et qui, lorsqu'elles sont déjetées, sont fortement latéralisées vers la droite. Malgré le faible nombre de pièces identifiées avec certitude, une grande quantité de lamelles a dû être produite par cette chaîne opératoire.

\section{PRODUCTION LAMELLAIRE INDIFFÉRENCIÉE}

Le problème qui se pose dans cette industrie est la coexistence de grattoirs et de burins aurignaciens, ces deux types d'outils-nucléus étant susceptibles de produire des lamelles identiques : les lamelles indifférenciées de type $\mathrm{A}$. Ces dernières seront donc étudiées globalement.

Du point de vue de leurs dimensions, deux groupes semblent se détacher (fig. 15a) : de petites lamelles $(2 \mathrm{~mm}$ à $5 \mathrm{~mm}$ de largeur pour $10 \mathrm{~mm}$ à $20 \mathrm{~mm}$ de longueur) et des lamelles un peu plus grandes $(6 \mathrm{~mm}$ à $9 \mathrm{~mm}$ de largeur pour $15 \mathrm{~mm}$ à $25 \mathrm{~mm}$ de longueur). Cependant, cette différence n'est pas significative pour distinguer l'origine de lamelles, car nous avons vu plus haut que le comportement des lamelles issues des burins aurignaciens est identique. Cette différence de gabarit (qui n'apparaît que sur les pièces entières) semble en fait totalement artificielle. En effet, elle n'apparaît pas si l'on observe la largeur de l'ensemble des lamelles indifférenciées de type A (fig. 16), pour lequel il y a une dominance des lamelles de $2 \mathrm{~mm}$ à $5 \mathrm{~mm}$ de largeur.

La concentration la plus importante (lamelles les plus petites) se superpose en grande partie au nuage 


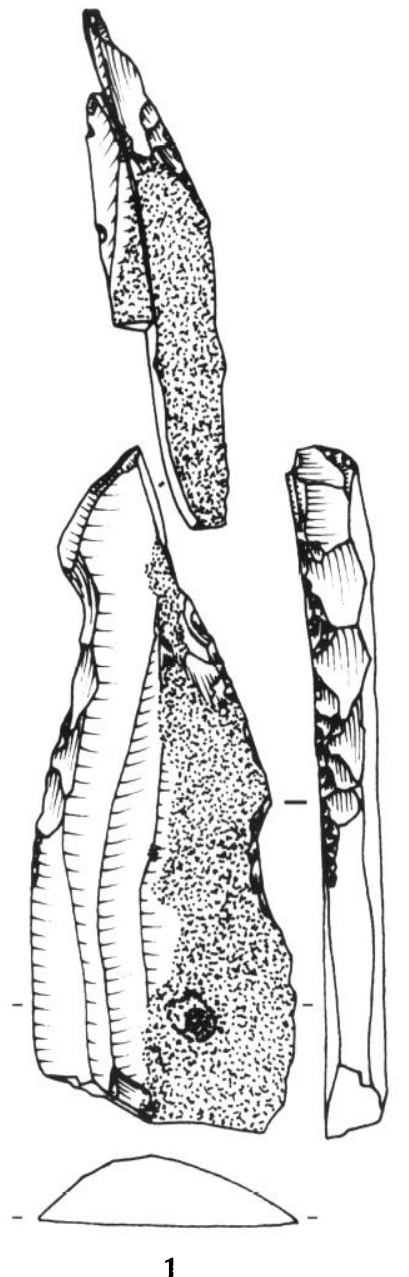

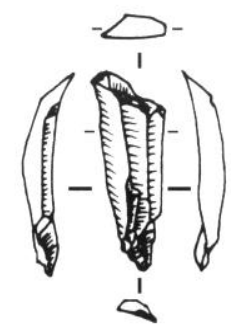

2

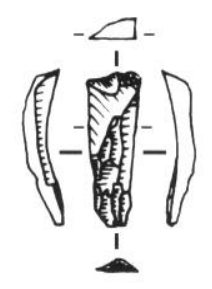

4

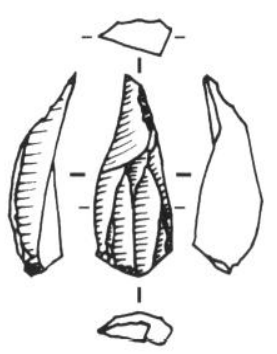

6

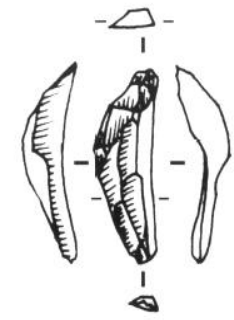

3

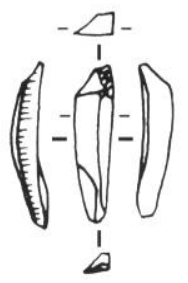

5

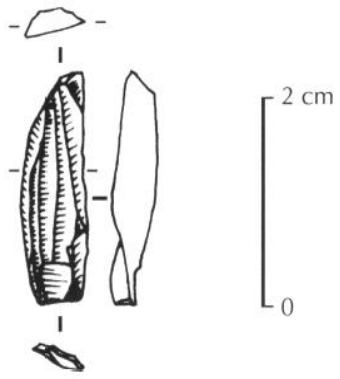

7

Fig. 20 - Éléments de la chaîne opératoire de production de lamelles de type C à partir des burins aurignaciens du niveau $7:$ Lower. 1, burin et ses chutes; $2-5$, lamelles de type $C ; 6$, lamelle de ravivage de la table de débitage ; 7 grande lamelle accidentelle (dessins $L$. Chiotti).

de points représentant les dimensions des négatifs présents sur les burins aurignaciens (fig. 15c). Ceci nous incite à penser que la plupart des pièces constituant la partie basse du nuage sont issues des burins aurignaciens.

La majorité des lamelles de type A ne sont pas déjetées $(57,41 \%)$. Cependant, les lamelles déjetées le sont essentiellement vers la droite $(24,07 \%$, pour seulement $6,48 \%$ vers la gauche). Les lamelles courbes et torses dominent avec $63,06 \%$. Les autres formes de lamelles sont toutes assez peu représentées, avec 17,12\% de lamelles courbes, $7,21 \%$ de lamelles rectilignes et $5,41 \%$ de lamelles torses. Trois types de lamelles se détachent donc de cet ensemble : des lamelles courbes et torses non déjetées (31 pièces), des lamelles courbes et torses déjetées à droite (27 pièces), et des lamelles courbes non déjetées (22 pièces).
Mis à part les observations effectuées directement sur les lamelles, le principal argument permettant de penser que la plupart d'entre elles sont issues des burins est le rapport entre les quantités d'outils-nucléus, qui est de 22 grattoirs-nucléus pour 80 burins-nucléus (voire même 97 biseaux).

En définitive, il ne ressort pas de réelle standardisation dans ce groupe de lamelles, ce qui semble normal étant donné qu'il s'agit d'un mélange de pièces de différentes origines. De plus, l'étude ne nous a pas permis de trouver des arguments permettant de distinguer réellement les lamelles des grattoirs de celles des burins. Cependant, du fait du rapport entre le nombre de burins et le nombre de grattoirs, il est probable que la majorité d'entre elles soient issues des burins, notamment les plus petites, comme le laissent penser les répartitions sur les graphiques longueur/largeur. 


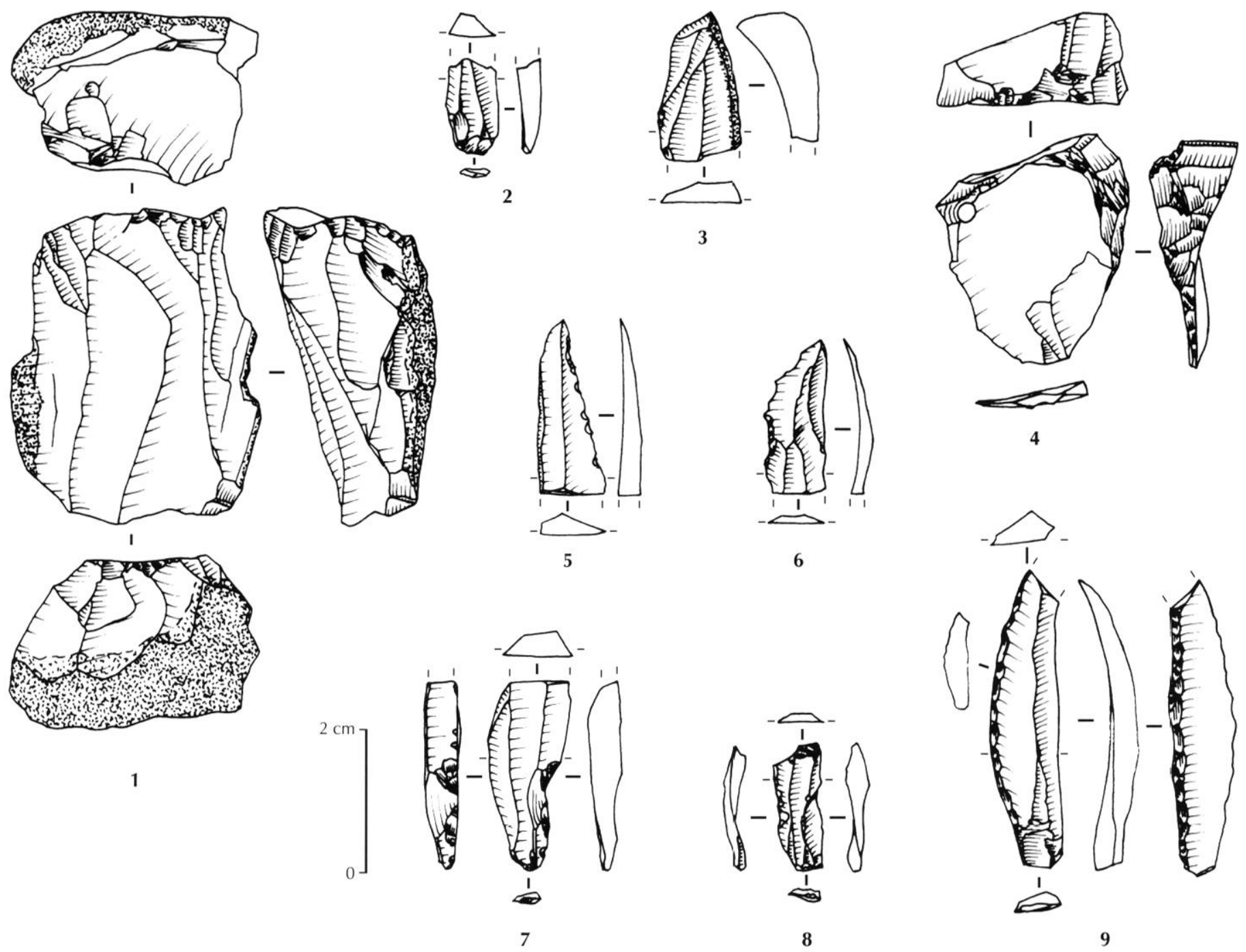

Fig. 21 - Production des nucléus prismatiques et outillage lamellaire du niveau $7:$ Lower. 1, nucléus laminaire; 2, 3, lamelles provenant du nucléus $n^{\circ} 1 ; 4$, tabletle de ravivage; $5,6,8$, lamelles retouchées; 7, lamelle à encoche; 9, lamelle Dufour (dessins L. Chiotti).

\section{PRODUCTION LAMELLAIRE} DES NUCLÉUS PRISMATIQUES

Les lamelles de type $\mathrm{D}$ provenant des nucléus prismatiques sont au nombre de 31 dans cette industrie, soit $9,34 \%$ des lamelles. Elles ont une largeur importante puisque treize d'entre elles font entre $11 \mathrm{~mm}$ et $12 \mathrm{~mm}$, et vingt-quatre entre $9 \mathrm{~mm}$ et $12 \mathrm{~mm}$. Ce sont toutes des lamelles non déjetées (sauf une pour laquelle ce caractère est indéterminé). La plupart sont rectilignes (18 pièces), mais toutes les autres formes sont représentées, notamment les lamelles torses avec 5 pièces.

Nous n'avons retrouvé ni de vrais nucléus prismatiques à lamelles, ni de nucléus laminaires réduits, dans cette industrie. Le débitage prismatique est néanmoins illustré par une petite tablette de ravivage qui porte des négatifs lamellaires (fig. 21, $\mathrm{n}^{\circ} 4$ ). Cependant, il est impossible de dire si cette tablette provient d'un vrai nucléus à lamelles ou d'un nucléus laminaire réduit.

La production de lamelles intercalées dans des schémas de productions laminaires peut également être illustrée dans cette industrie. Pour cela, nous prendrons l'exemple du nucléus laminaire (fig. $21, \mathrm{n}^{\circ} 1$ ) qui a fourni la lame sur laquelle est réalisé le burin busqué dont nous avons parlé plus haut afin d'illustrer la quantité de lamelles produites par ces burins-nucléus (fig. 20, $\mathrm{n}^{\circ} 1$ ). Comme nous l'avons déjà signalé, la matière première employée est un silex du Bergeracois très reconnaissable, notamment par sa zone souscorticale de couleur brune et jaune. Malgré ses faibles 
dimensions lors de son abandon $(4.5 \mathrm{~mm}$ x $36 \mathrm{~mm}$ x $22 \mathrm{~mm}$ ), ce nucléus prismatique bipolaire a fourni des lames de grandes dimensions puisque celle qui sert de support au burin busqué fait déjà plus de $10 \mathrm{~cm}$ (en tenant compte de la réduction que nous avons pu identifier) alors qu'elle est cassée. Trois autres lames de dimensions moindres, mais néanmoins importantes, ont été identifiées. Nous avons également retrouvé deux segments de lamelles, dont une est outrepassée (fig. 21, $n^{\prime \prime} 2,3$ ), qui proviennent de ce même bloc. Nous ne sommes en présence que d'une faible quantité de pièces (11 au total) et, en l'absence de possibilités de remontage, il nous est impossible de préciser la position de ces lamelles dans la séquence de débitage. Cependant ce qui importe ici est de constater que ce nucléus a fourni d'une part des grandes lames, et d'autre part des lamelles.

Dans ce cas particulier, nous pouvons exclure une réduction du nucléus dans le but d'obtenir des lamelles car les derniers enlèvements visibles ont des dimensions de petites lames.

Pour cette industrie, nous n'excluons pas la possibilité d'une production de lamelles par des nucléus à lamelles ou des nucléus à lames réduits, mais nous n'en avons aucune preuve.

\section{LAMELLES OPPORTUNISTES}

Elles ne sont représentées que par 18 pièces, soit $5,42 \%$ des lamelles. Il s'agit essentiellement de pièces rectilignes et non déjetées. Ce sont des lamelles de dimensions asse $\%$ importantes, avec des largeurs comprises entre $8 \mathrm{~mm}$ et $12 \mathrm{~mm}$ et des longueurs entre $20 \mathrm{~mm}$ et $35 \mathrm{~mm}$ (fig. 15a). Leur rapport longueur/ largeur forme un nuage très diffus. Elles forment en fait un groupe sans aucune homogénéité.

Cés lamelles aux formes très irrégulières sont des sous-produits qui peuvent provenir de n'importe quel type de débitage.

\section{OUTILLAGE LAMELLAIRE}

L'outillage lamellaire est peu développé dans l'industrie du niveau $7:$ I.ower. Seules 14 lamelles ont été transformées en outils: 6 en lamelles retouchées, 5 en lamelles à encoche, 2 en lamelles tronquées et 1 en lamelle Dufour.
Les supports choisis sont très rariés, et ne proviennent manifestement pas d'une volonté de produire des supports afin de les transformer en outils, mais plutôt d'un choix aléatoire de certaines pièces lorsqu'un besoin se présentait.

Les supports utilisés pour les différents outils sont les suivants :

- lamelles retouchées : 2 lamelles de type B, 2 lamelles de type $\mathrm{D}, 1$ lamelle de type $\mathrm{C}$ et 1 lamelle indifférenciée (type A) ;

- lamelles à encoche : 2 lamelles de type B, 1 lamelle de type D, 1 lamelle indifférenciée (type A) et 1 lamelle indéterminée ;

- lamelles tronquées: 1 lamelle de type D et 1 lamelle indéterminée ;

- lamelle Dufour : 1 lamelle indifférenciée (type A).

À l'exception de la lamelle Dufour (fig. 21, n 9), tous ces outils sont en fait très peu retouchés. Les aménagements, qu'il s'agisse d'encoches, de troncatures ou de bords retouchés, ne comportent que quelques retouches qui, de plus, ne sont pas d'une grande régularité (fig. 21, $\left.\mathrm{n}^{\circ \prime} 5-8\right)$.

La lamelle Dufour est en revanche très fortement retouchée. Il s'agit d'une lamelle à retouche alterne: inverse sur le bord droit et directe sur le bord gauche. La retouche inverse est abrupte, très marquée et présente sur toute la longueur de la lamelle. La retouche directe est moins importante, mais elle est également continue sur toute la longueur.

Le support est une lamelle indifférenciée de petites dimensions $(13,7 \mathrm{~mm} \times 3,4 \mathrm{~mm} \times 1,4 \mathrm{~mm})$, ce qui la place exactement dans le gabarit dominant des lamelles produites sur les burins aurignaciens.

Malgré la quantité de lamelles produites, cette lamelle Dufour est la seule qui ait été retrouvée. Même si une partie des lamelles ont dû être utilisées brutes, il paraît étonnant de ne trouver qu'un seul exemplaire de ce type d'outils dans une telle industrie. Ces lamelles étant de très petites dimensions, nous pensons qu'il est possible qu'une partie d'entre elles n'aient pas été récoltées.

Lors de récents travaux à Caminade, J.-G. Bordes et A. Lenoble (2001) ont identifié, dans l'Aurignacien récent, de petites lamelles retouchées qui sont en fait des chutes de burins busqués portant une retouche directe (ou trace d'utilisation) sur le dos de la chute. Ces lamelles retouchées sont de très petites dimensions : en 
moyenne $15 \mathrm{~mm}$ x $3 \mathrm{~mm}$ x $3 \mathrm{~mm}$ (Bordes, Lenoble, 2002 ; Bordes, à paraître).

Cette observation conforte notre première idée selon laquelle il est possible que la fraction la plus fine n'ait pas été recueillie, d'autant plus qu'à Caminade, ces pièces n'ont pas été vues lors des anciennes fouilles de B. Mortureux et D. de Sonneville-Bordes (SonnevilleBordes, Mortureux, 1955 ; Sonneville-Bordes, 1970). Par conséquent, si de telles chutes de burins retouchées étaient présentes, elles ont disparu lors de la fouille.

En conclusion, l'outillage lamellaire du niveau 7 : Lower est constitué de quelques pièces assez peu retouchées dont les supports sont choisis de façon aléatoire parmi les pièces disponibles.

La chaîne opératoire de production de lamelles à partir des burins aurignaciens a permis d'obtenir des pièces de très faibles dimensions qui pouvaient être destinées soit à être retouchées (pièces non retrouvées?), soit à être utilisées brutes. La présence d'une lamelle Dufour très finement retouchée et de faibles dimensions pourrait être un argument en faveur de la première de ces deux hypothèses.

\section{NIVEAUX 7 : UPPER ET 6 , AURIGNACIEN ÉVOLUÉ}

Les niveaux 7 : Upper et 6 (et plus particulièrement le niveau 6) présentent des industries assez particulières, dans le sens où la majeure partie des outils ont un aspect assez irrégulier. Ceci concerne la portion de l'outillage que nous avons dénommée "outils peu élaborés " (pièces plus ou moins retouchées, pièces à encoche ou denticulés), mais également les "outils élaborés " (grattoirs, burins...). Cette irrégularité des pièces, assez difficile à quantifier, laisse dans l'ensemble l'impression d'industries frustes dont les pièces semblent avoir été réalisées rapidement, sans réelle volonté d'obtenir de «beaux "supports, comme c'est le cas dans les industries des autres niveaux (Chiotti, 1999).

En plus de cet aspect fruste, il faut signaler, dans le niveau 6 , un très fort taux de fracturation, en particulier pour les lamelles, puisque $88,67 \%$ de ces dernières sont fracturées (alors que ce taux ne dépasse jamais $72 \%$ dans les autres niveaux). De plus, il s'agit souvent d'une fracturation multiple puisque $53,30 \%$ des fragments sont mésiaux, le plus souvent courts. Cette remarque permet d'expliquer le fort taux de lamelles indéterminées ainsi que le nombre important de pièces pour lesquelles il nous a été impossible de déterminer certains caractères, tels que la forme ou la déviation.

Le niveau 7 : Upper a livré 115 lamelles (tabl. I), dont : 31 lamelles indifférenciées de type A, soit $26,96 \%$;

29 lamelles de type B, soit $25,22 \%$;

24 lamelles de type C, soit $20,87 \%$;

12 lamelles de type D, soit $10,43 \%$;

7 lamelles opportunistes, soit $6,09 \%$;

12 lamelles indéterminées soit $10,43 \%$.

Le niveau 6 a livré 259 lamelles dont :

64 lamelles indifférenciées de type A, soit $24,71 \%$;

68 lamelles de type B, soit $26,25 \%$;

5 lamelles de type $\mathrm{C}$, soit $1,93 \%$;

35 lamelles de type $\mathrm{D}$, soit $13,51 \%$;

17 lamelles opportunistes, soit $6,56 \%$;

70 lamelles indéterminées, soit $27,03 \%$.

Contrairement aux autres industries, le silex importé de la région de Bergerac a été fortement employé pour réaliser les lamelles du niveau 6. En effet, sur les 259 lamelles, 41 sont en silex du Bergeracois, soit 15,83\%, alors que ce dernier ne représente que $5,71 \%$ de l'ensemble de la production lithique de ce niveau. Comme c'est généralement le cas, il est mieux représenté dans l'ensemble de l'outillage $(9,83 \%)$, et encore plus dans l'outillage lamellaire $(24,44 \%)$. Cette matière n'a généralement pas été débitée sur place, cependant, dans le niveau 6, nous avons retrouvé deux nucléus (dont un portant quelques négatifs lamellaires, cf. p. 148) et une quantité de produits de débitage bruts supérieure à celle des autres niveaux, ce qui atteste un débitage dans l'abri. Cette matière première est présente dans tous les types de production de lamelles puisqu'elle a fourni 10 lamelles de type $\mathrm{B}, 10$ lamelles de type $\mathrm{D}, 10$ lamelles indéterminées, 7 lamelles indifférenciées de type A et 4 lamelles opportunistes.

\section{PRODUCTION LAMELLAIRE DES GRATTOIRS CARÉNÉS ET À MUSEAU}

\section{LES GRATTOIRS-NUCLÉUS}

Dans le niveau 7: Upper, les grattoirs aurignaciens sont représentés par 14 pièces, soit $14,43 \%$ de l'outillage. Il s'agit de 9 grattoirs carénés (dont 3 atypiques) et de 5 grattoirs à museau épais. Leurs supports sont 
constitués de 11 éclats, 2 blocs et 1 lame. Quatre d'entre cux présentent une encoche, toujours sur le bord gauche du front. Parmi les carénés, quatre sont des carénés à coches (Ronen, 1964). Deux ravivages de grattoirs carénés (Chiotti, 2000) sont présents, tous deux détachés sur le bord gauche des fronts.

Dans le niveau 6, nous avons 17 grattoirs aurignaciens, ce qui représente $2,35 \%$ de l'outillage. Il s'agit de 13 grattoirs carénés et 4 grattoirs à museau. Leurs supports sont constitués de 6 éclats, 6 blocs et 1 lame. Deux grattoirs ont une encoche de part et d'autre du front, 2 en ont une sur le bord gauche et 1 en a une sur le bord droit. Cinq ravivages de grattoirs carénés sont présents dont 4 détachés sur le bord gauche et 1 sur le bord droit.
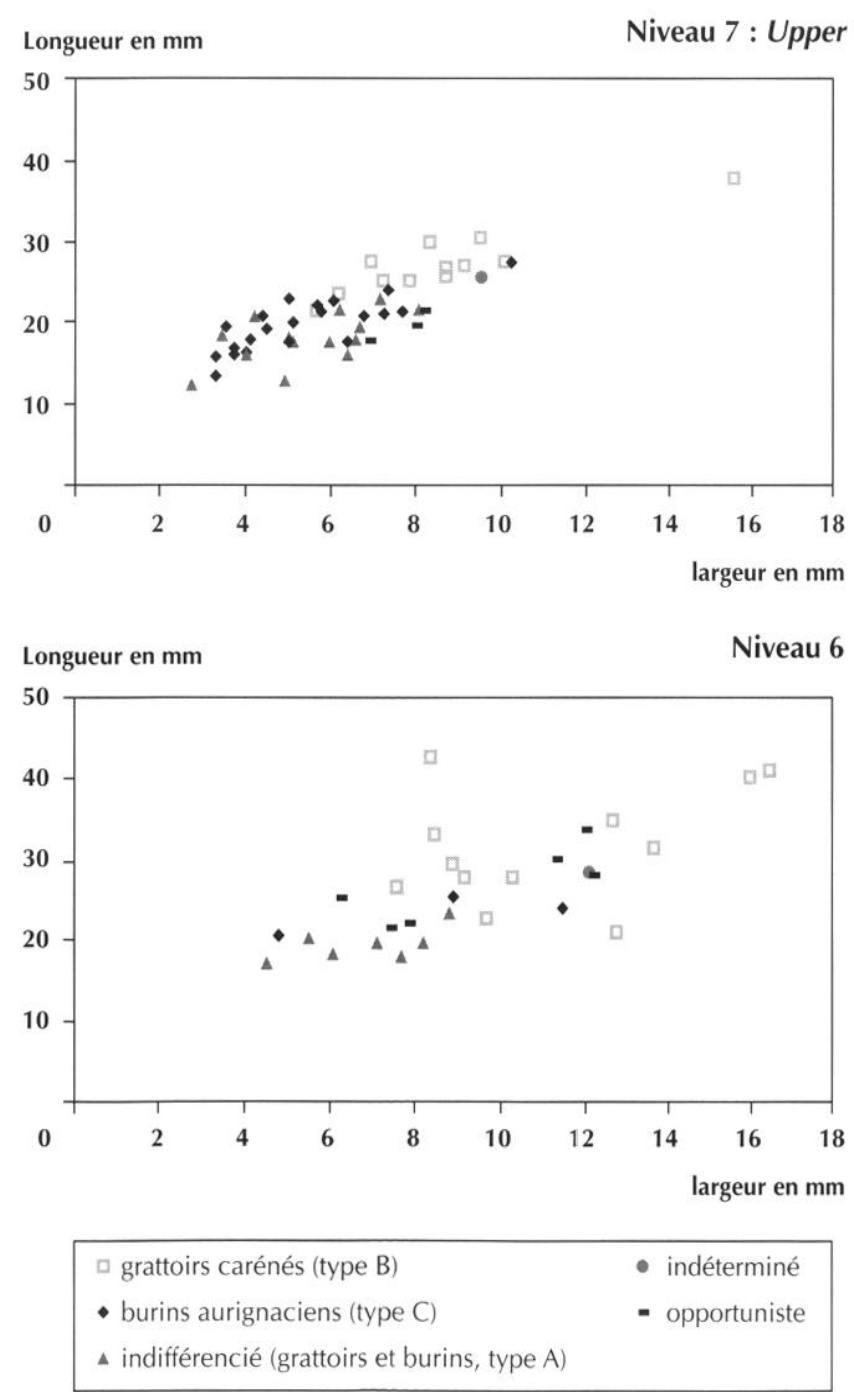

Fig. 22 - Diagrammes présentant les rapports longueur/largeur des différents types de lamelles entières, dans les niveaux 7 : Upper et 6.

\section{LES LAMELLES DE TYPE B}

Les lamelles de type B sont au nombre de 29 dans le niveau 7: upper et de 68 dans le niveau 6. Les pièces entières sont de dimensions plus homogènes dans le niveau 7: Upper (fig. 22). Les largeurs de la totalité de ces lamelles donnent des résultats similaires pour les deux niveaux, avec des pièces dont la largeur est essentiellement comprise entre $7 \mathrm{~mm}$ et $12 \mathrm{~mm}$ (fig. 23). La plupart de ces lamelles ne sont pas déjetées, et parmi celles qui le sont, il y en a une majorité à droite. Dans le niveau 7: Upper, il y a une dominance des lamelles courbes et torses, avec 14 pièces sur 29; en revanche, dans le niveau 6, il n'y a aucune forme préférenticlle, avec autant de pièces courbes, que de torses et de courbes et torses.

Comme dans les niveaux précédemment étudiés, les lamelles de type B ne sont donc pas standardisées, en particulier dans le niveau 6 où elles présentent une grande variété, quel que soit le caractère considéré. Comme cela a déjà été vu plus haut, ces lamelles ne sont en fait que des phases de préparation. De plus, la grande variété de formes du niveau 6 est sans doute liée à l'un des caractères principaux de cette industrie, à savoir une grande irrégularité de l'ensemble des pièces qui se traduit par un aspect général très fruste, quel que soit le type de pièces observé.

\section{PRODUCTION LAMELLAIRE DES BURINS AURIGNACIENS}

\section{LES BURINS-NUCLÉUS}

Les burins aurignaciens du niveau 7 : Upper sont au nombre de cinq : 3 burins busqués, 1 burin des Vachons et 1 burin caréné (sur un burin multiple mixte). Les plans de frappe sont formés soit par un ou deux coups de burin (respectivement 2 et 1 pièces), soit par un pan naturel (1 pièce). La table de débitage est sur la gauche pour quatre biseaux et sur la droite pour le cinquième.

Les burins aurignaciens sont au nombre de 30 dans le niveau 6 (dont 1 sur un burin multiple mixte et 3 sur des grattoirs-burins). Il s'agit de 12 burins busqués (dont 2 atypiques), 9 burins carénés et 9 burins des Vachons. Ils sont réalisés sur 14 lames et 16 éclats. Dix-huit burins sont en partie distale des supports et douze en partie proximale. Ils sont fortement latéralisés, avec 24 pièces 
lamelles des grattoirs carénés (type B)

nombre de pièces

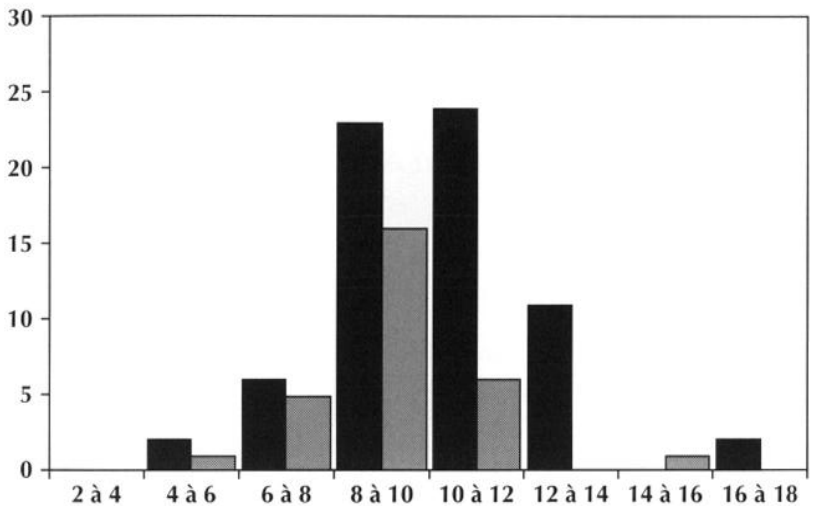

largeur en mm

lamelles indifférenciées (grattoirs et burins, type A)

nombre de pièces

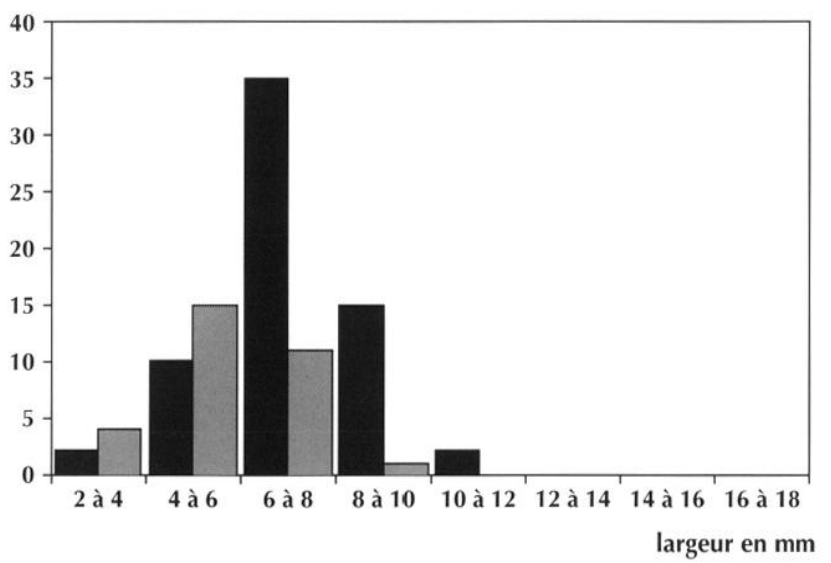

lamelles des burins aurignaciens (type C)

nombre de pièces

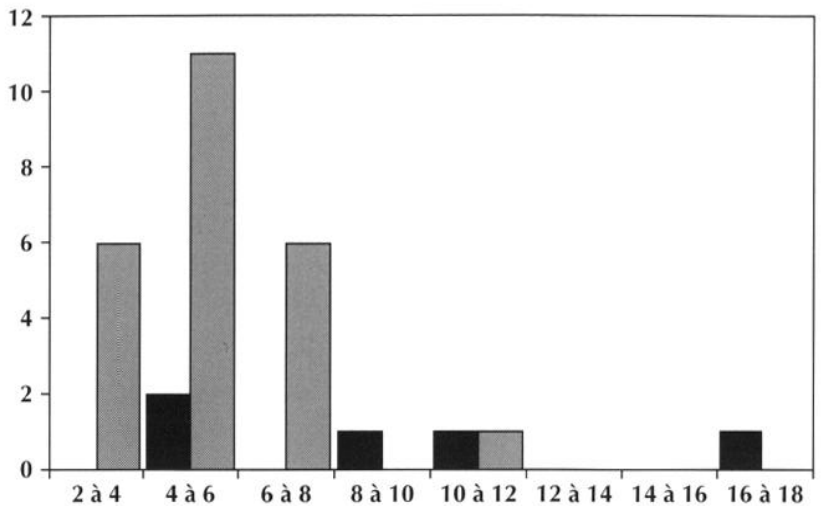

largeur en $\mathrm{mm}$

lamelles des nucléus prismatiques (type D)

nombre de pièces

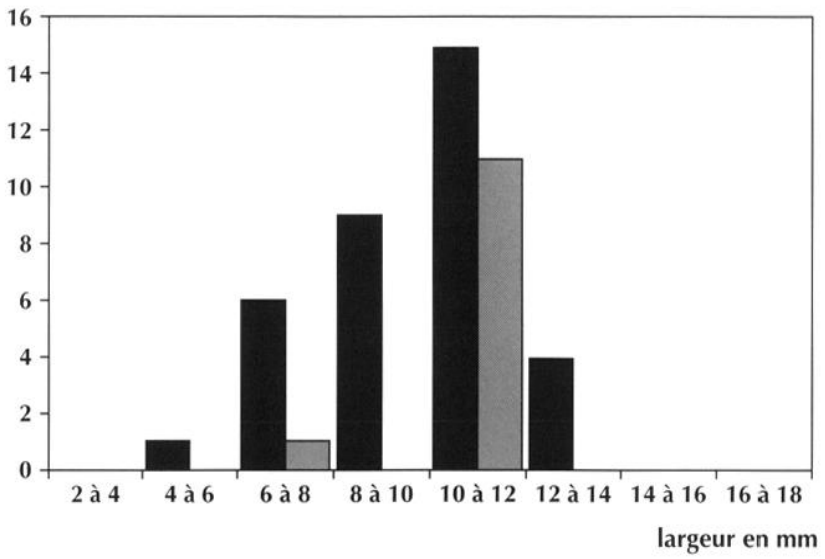

niveau 6

niveau 7 : Upper

Fig. 23 - Histogrammes présentant les largeurs des lamelles des grattoirs carénés (type B), des lamelles des burins aurignaciens (type (), des lamelles indifférenciées (type A) et des lamelles des nucléus prismatiques (type D) dans les niveaux $7:$ Upper et 6.

dont le pan secondaire est sur la gauche et sculement 6 sur la droite.

\section{LES LAMELLES DE TYPE C}

Les lamelles de type (i sont assez peu nombreuses avec 24 pièces dans le niveau 7 : Upper, et seulement 5 dans le niveau 6.

Dans le niveau 7 : (ppper, elles ont des largeurs asse\% variables (fig. 22), comprises entre $3 \mathrm{~mm}$ et $8 \mathrm{~mm}$ (fig. 23). Les longueurs sont quant à elles comprises entre $15 \mathrm{~mm}$ et $25 \mathrm{~mm}$. La majorité de ces lamelles (16 sur 24) sont déjetées à droite. Elles sont presque toutes courbes et torses (22 sur 24). La facette abrupte se situe presque toujours sur le bord droit (22 lamelles sur 24).

Dans le niveau 6 le nombre de pièces est trop faible pour tenter une quelconque analyse. La seule remarque possible est que les 5 lamelles sont toutes courbes et torses.

\section{PRODUCTION LAMELLAIRE INDIFFÉRENCIÉE}

Comme dans le niveau $7:$ Lower, nous avons dans ces industries une coexistence de grattoirs et de burins aurignaciens, et donc une partie des lamelles de type A dont l'origine est indifférenciée. 
Il y a trop peu de pièces entières dans ces industries pour obtenir des données à partir des rapports longueur/largeur, en particulier dans le niveau 6 (fig. 22). Les largeurs de l'ensemble des lamelles indifférenciées donnent des résultats légèrement différents dans les deux industries, avec un pic entre $4 \mathrm{~mm}$ et $7 \mathrm{~mm}$ pour le niveau 7 : Upper et entre $6 \mathrm{~mm}$ et $9 \mathrm{~mm}$ pour le niveau 6 (fig. 23).

Ces lamelles sont donc en moyenne plus petites dans le niveau 7 : Upper. Ceci est peut-être lié au fait qu'une partie plus importante d'entre elles doit provenir de burins aurignaciens. En effet, ces derniers sont plus nombreux dans cette industrie que les grattoirs aurignaciens (respectivement 31 et 17 pièces), et nous avons vu dans les autres niveaux que les lamelles de type $\mathrm{A}$ issues des burins aurignaciens sont généralement sensiblement plus petites que celles issues des grattoirs (cf. p. 141). Au contraire dans le niveau 6 , qui ne contient que très peu de burins aurignaciens ( 5 pièces), ces lamelles sont en grande majorité issues des grattoirs (17 pièces), et sont en moyenne un peu plus grandes.

La majorité des lamelles indifférenciées ne sont pas déjetées, mais dans les deux niveaux il y a quand même un nombre important de lamelles déjetées à droite. Il faut également signaler que dans le niveau 6 la catégorie dominante est celle des indéterminés, ce qui est lié à la très forte fracturation de cette industrie. Dans le niveau $7:$ Upper, la majorité des pièces sont courbes et torses. En revanche dans le niveau 6 , il n'y a aucune forme dominante, avec quasiment autant de pièces rectilignes, courbes, torses ou courbes et torses.

En définitive, nous n'avons pas pu mettre en évidence de type et de gabarit de lamelles vraiment dominants, sans doute essentiellement en raison de la quantité limitée de pièces.

Quant à l'origine de ces lamelles, la seule chose que nous pouvons dire est liée aux quantités de burins de pièces nucléiformes. Elles proviendraient plutôt des burins dans le niveau $7:$ Upper et plutôt des grattoirs dans le niveau 6 , ceci semblant confirmé par les dimensions des pièces.

\section{PRODUCTION LAMELLAIRE DES NUCLÉUS PRISMATIQUES}

Les lamelles de type D sont au nombre de 12 dans le niveau 7 : Upper et de 35 dans le niveau 6. Ce sont des lamelles assez larges: entre $10 \mathrm{~mm}$ et $12 \mathrm{~mm}$ dans le niveau 7 : Upper et entre $7 \mathrm{~mm}$ et $13 \mathrm{~mm}$ dans le niveau 6 (fig. 23). Parmi les pièces pour lesquelles la détermination de ces caractères a été possible, aucune n'est déjetée, et presque toutes sont rectilignes.

Comme dans les autres niveaux, plusieurs modalités de production sont envisageables : des lamelles produites à partir de nucléus à lamelles, des lamelles produites à partir de nucléus à lames réduits ou des lamelles intercalées dans une production laminaire.

Ce dernier cas n'a pas pu être mis en évidence dans ces deux niveaux. En revanche, les deux premiers sont représentés et peuvent être identifiés grâce à la présence de nucléus et de tablettes de ravivage.

Les nucléus laminaires réduits jusqu'à la production de lamelles sont représentés par trois pièces dans le niveau 6 : deux nucléus prismatiques bipolaires et un nucléus prismatique unipolaire.

Le plus gros nucléus prismatique du niveau 6 (52 $\mathrm{mm} \times 34 \mathrm{~mm} \times 33 \mathrm{~mm}$ ) présente de nombreux négatifs de lamelles (fig. $24, \mathrm{n}^{\circ} 1$ ). Il s'agit d'un nucléus pour lequel nous avons retrouvé une tablette de ravivage (fig. 24, $\mathrm{n}^{\circ} 2$ ). Cette dernière, qui porte également des négatifs de lamelles, ne remonte pas directement sur le plan de frappe du nucléus, mais sa position peut être déterminée grâce à sa forme et à la présence de cortex. Cette position indique qu'un nombre relativement important de lamelles a dû être obtenu à partir de ce nucléus. La forme et les dimensions de ce nucléus nous ont fait penser, dans un premier temps, qu'il s'agissait d'un nucléus laminaire réduit. Cependant la position de la tablette (qui révèle la quantité importante de lamelles produites) nous incite à penser que cette pièce pourrait en fait être un nucléus à lamelles stricto sensu. La matière très particulière de cette pièce nous permet, de plus, d'affirmer qu'aucune des lamelles produites n'a été retrouvée sur le site.

En revanche, les deux autres nucléus du niveau 6 ont fourni nettement moins de lamelles puisque des négatifs de lames sont encore visibles et que seuls les derniers enlèvements sont des lamelles.

Le premier ( $54 \mathrm{~mm}$ x $32 \mathrm{~mm}$ x $27 \mathrm{~mm}$ ) est un nucléus prismatique unipolaire en silex sénonien noir. Il a donné quelques lamelles qui ont toutes rebroussé sur une irrégularité située au centre de la surface de débitage (fig. $24, n^{\circ} 3$ ). Les négatifs précédents encore visibles sont laminaires. 


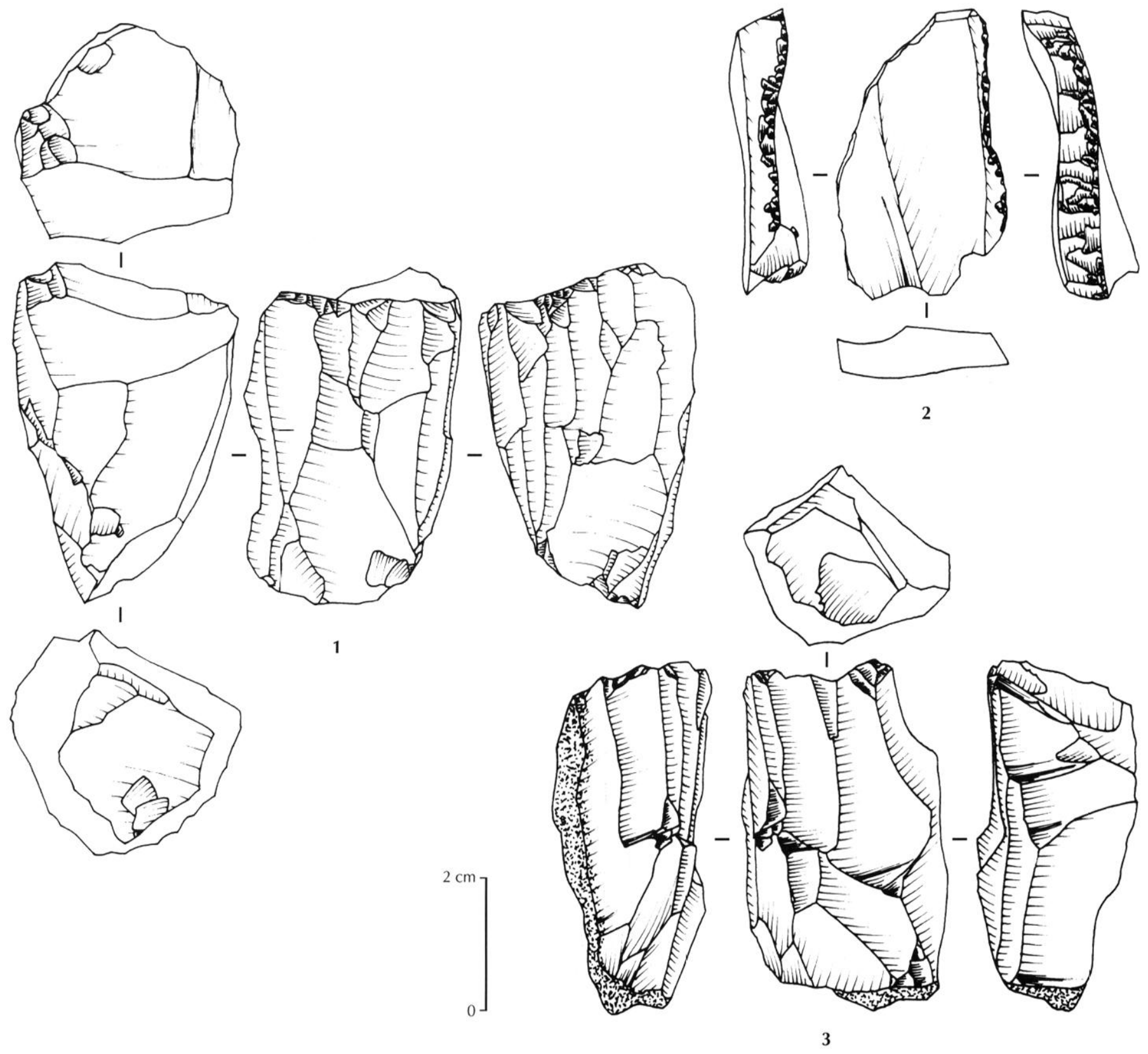

Fig. 24 - Nucléus et tablette de ravivage du niveau $6: 1$, nucléus prismatique pouvant être un nucléus à lamelles stricto sensu ; 2 , tablette de ravivage provenant du nucléus $n^{\circ} 1 ; 3$, nucléus prismatique laminaire réduit (dessins $L$. Chiotti).

Le second (42 mm x $37 \mathrm{~mm} \times 32 \mathrm{~mm}$ ) est l'un des rares nucléus en silex importé de la région de Bergerac. Il est bipolaire, à deux plans de frappe croisés (fig. 25, $\mathrm{n}^{\circ} 1$ ). Seuls les derniers enlèvements de l'un des plans de frappe sont de dimensions lamellaires.

Dans ces deux derniers cas, l'obtention de lamelles semble plus opportuniste que due à une réduction volontaire dans le but de débiter des lamelles.
Le niveau 6 a également livré un vrai nucléus à lamelles. Celui-ci est réalisé sur un petit galet de silex sénonien noir, qui n'est autre qu'un ancien éclat, par la suite roulé par la rivière (fig. $25, \mathrm{n}^{\circ} 2$ ). Cette pièce, de très faibles dimensions ( $40 \mathrm{~mm} \times 31 \mathrm{~mm} \times 17 \mathrm{~mm}$ ), est le résultat d'un débitage développé sur un galet qui ne devait pas être beaucoup plus gros. Deux plans de frappe successifs et opposés ont été aménagés afin d'exploiter 

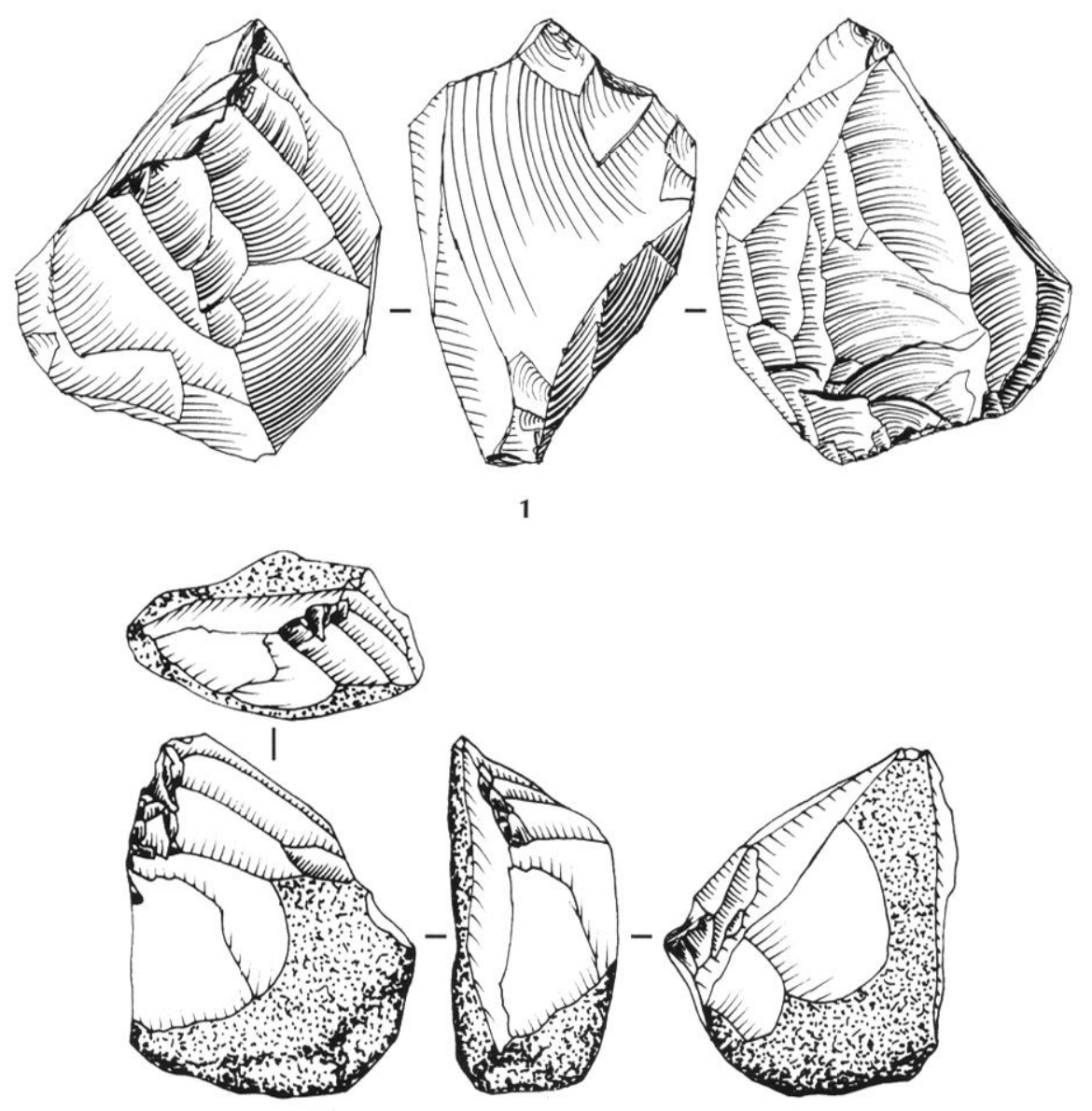

2

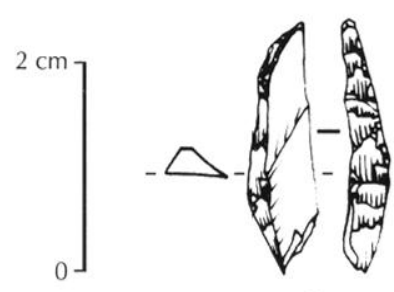

3

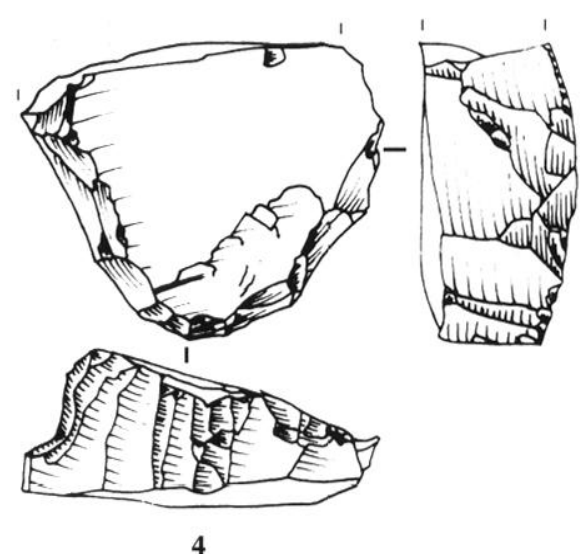

Fig. 25 - Nucléus et tablettes de ravivage du niveau $6: 1$, nucléus laminaire réduit en silex du Bergeracois; 2 , nucléus à lamelles stricto sensu ; 3, tablette de ravivage partiel du plan de frappe; 4, tablette de ravivage total du plan de frappe (dessins : 1, M. Dauvois ; 2-4, L. Chiotti).

l'épaisseur de la tranche du galet. Le dernier, qui a permis de détacher les lamelles dont les négatifs sont identifiables, a été réalisé simplement par le détachement d'un éclat laminaire ou lamellaire. D'après les négatifs, les lamelles obtenues sont des pièces courbes et torses, déjetées à droite, qui n'ont pas l'aspect de lamelles issues de nucléus, mais sont plutôt semblables à des lamelles issues de grattoirs carénés.

En plus de ces nucléus relativement bien identifiés, nous avons retrouvé deux pièces de très faibles dimensions pour lesquelles il n'a pas été possible de dire s'il s'agissait de petits nucléus à lamelles ou de réductions 
extrêmes de nucléus à lames. L'une provient du niveau 7: Upper ( $40 \mathrm{~mm} \times 22 \mathrm{~mm} \times 19 \mathrm{~mm}$ ) et l'autre du niveau $6(31 \mathrm{~mm} \times 31 \mathrm{~mm} \times 17 \mathrm{~mm})$. Ces pièces portent quelques négatifs lamellaires, mais leur état d'exhaustion final ne permet pas de trancher.

Le niveau 6 a également livré quatre tablettes de ravivage (en plus de celle correspondant au nucléus, citée plus haut) qui portent des traces de négatifs de lamelles. Il s'agit de 3 tablettes à section plate qui ravivent la totalité du plan de frappe (fig. $25, \mathrm{n}^{\circ} 4$ ), et d'une tablette à section triangulaire et de forme lamellaire qui ne ravive que partiellement le plan de frappe (fig. 25, $n^{\circ} 3$ ) (Inizan et al., 1995). Elles attestent un débitage prismatique de lamelles, mais il est impossible de dire si elles proviennent de vrais nucléus à lamelles ou de nucléus à lames réduits.

\section{LAMELLES OPPORTUNISTES}

Les lamelles opportunistes sont très peu nombreuses avec seulement sept pièces dans le niveau $7:$ Upper et dixsept dans le niveau 6. Comme dans les autres niveaux, il s'agit de lamelles irrégulières qui n'ont aucun caractère permettant de les rattacher à l'un ou l'autre des groupes précédents et qui ne présentent pas de standardisation.

\section{OUTILLAGE LAMELLAIRE}

Dans le niveau 7 : Upper, l'outillage sur lamelles est très peu présent, avec seulement deux lamelles Dufour et une lamelle retouchée sur un bord. L'outillage sur lamelles est en revanche beaucoup plus développé dans le niveau 6 . Mis à part le niveau 8 et sa grande quantité de lamelles Dufour, c'est celui qui contenait le plus de lamelles transformées avec 45 pièces. Cet outillage est composé de 22 lamelles plus ou moins retouchées, 10 lamelles de Font-Yves, 4 lamelles Dufour, 4 lamelles à encoche, 2 lamelles denticulées, 2 lamelles tronquées et 1 bec.

La plupart de ces outils sont des pièces assez peu retouchées, de façon discontinue ou partielle, et généralement assez irrégulière. C'est surtout le cas pour les pièces plus ou moins retouchées, les lamelles à encoche, les lamelles denticulées, les lamelles tronquées, les petites lamelles Dufour et le bec (fig. 26, $\mathrm{n}^{\mathrm{os}}$ 9-11).

Ces dernières catégories d'outils sont, comme dans les niveaux précédents, réalisées sur des supports très variés qui ne résultent pas d'une sélection rigoureuse en fonction de leur forme. Ces outils s'intègrent tout à fait dans l'aspect général assez fruste du gros outillage réalisé sur lames et sur éclats.

Les supports utilisés pour réaliser ces outils sommaires sont les suivants :

- lamelles plus ou moins retouchées: 8 lamelles de type B, 4 lamelles de type D, 1 lamelle opportuniste, 1 lamelle indifférenciée (type A) et 8 lamelles indéterminées ;

- lamelles à encoche : 2 lamelles de type $\mathrm{D}, 1$ lamelle indifférenciée (type A) et 1 lamelle indéterminée ;

- lamelles denticulées : 2 lamelles de type $\mathrm{D}$;

- lamelles tronquées : 2 lamelles de type B ;

- petites lamelles Dufour (fig. 26, $n^{\circ} 6$ ) : 1 lamelle indifférenciée (type A) et 1 lamelle indéterminée ;

- bec: 1 lamelle de type $\mathrm{D}$.

Hormis ces pièces faiblement retouchées, le niveau 6 a également livré quelques outils de très bonne facture constitués de 10 lamelles de Font-Yves et 2 grandes lamelles Dufour.

Bien que toutes fragmentaires, les 10 pièces identifiées comme lamelles de Font-Yves sont très caractéristiques des pièces décrites sur le site de Font-Yves (Bouyssonie et al., 1913). Elles sont très standardisées, toutes réalisées sur des lamelles de type $\mathrm{D}$, de dimensions assez proches: entre $4,7 \mathrm{~mm}$ et $8,2 \mathrm{~mm}$ de largeur (fig. 26, $\mathrm{n}^{\mathrm{os}} 1-5$ ). Pour huit de ces pièces, la retouche est abrupte sur l'un des bords, formant parfois presque un petit dos. Comme c'est généralement le cas sur ce type de pièces (Demars, Laurent, 1989), le dos se situe sur le bord droit. Le bord opposé au dos porte généralement une retouche marginale directe, sauf dans un cas où elle est inverse. Il y a également deux pièces pour lesquelles le bord opposé n'est pas retouché.

Parmi ces lamelles, deux ont conservé une extrémité aménagée (fig. 26, $\mathrm{n}^{\circ} 2,5$ ). L'aménagement consiste en un rétrécissement de la lamelle par une retouche qui rend les deux bords convergents, jusqu'à former une sorte de petite pointe. Dans les deux cas, cet aménagement concerne l'extrémité proximale de la lamelle. Il semble plutôt s'agir d'une base que d'une vraie pointe.

Les deux grandes lamelles Dufour sont également fragmentaires. Il s'agit de parties proximales de grandes lamelles longues et étroites $(31,5 \mathrm{~mm} \times 4,2 \mathrm{~mm} \times 2,3 \mathrm{~mm}$ et $23,1 \mathrm{~mm} \times 5,2 \mathrm{~mm} \times 2,3 \mathrm{~mm}$ ). Ce sont des lamelles à profil courbe et torse, issues de grattoirs carénés (fig. 26, 


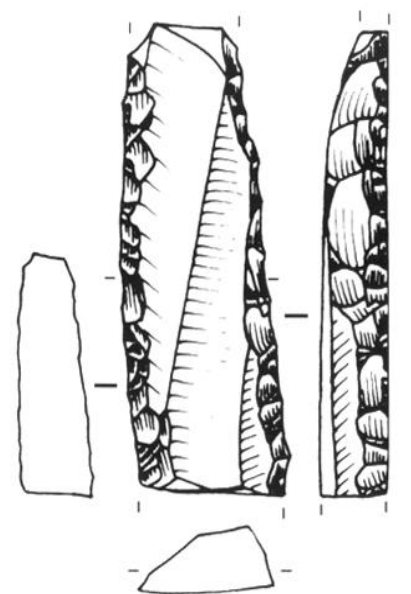

1

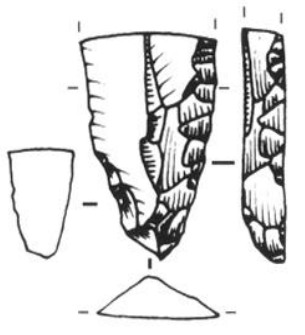

5

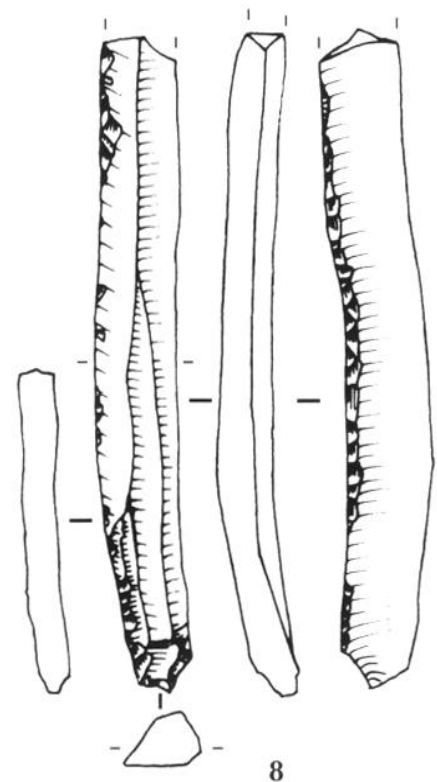

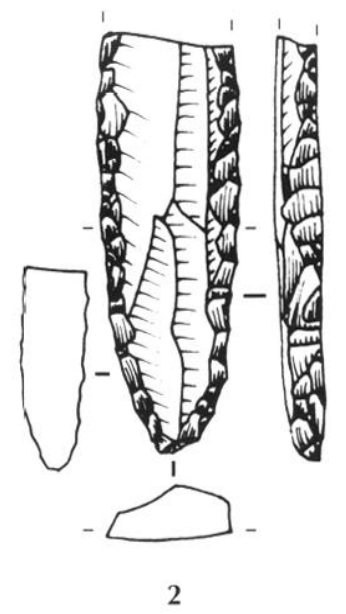
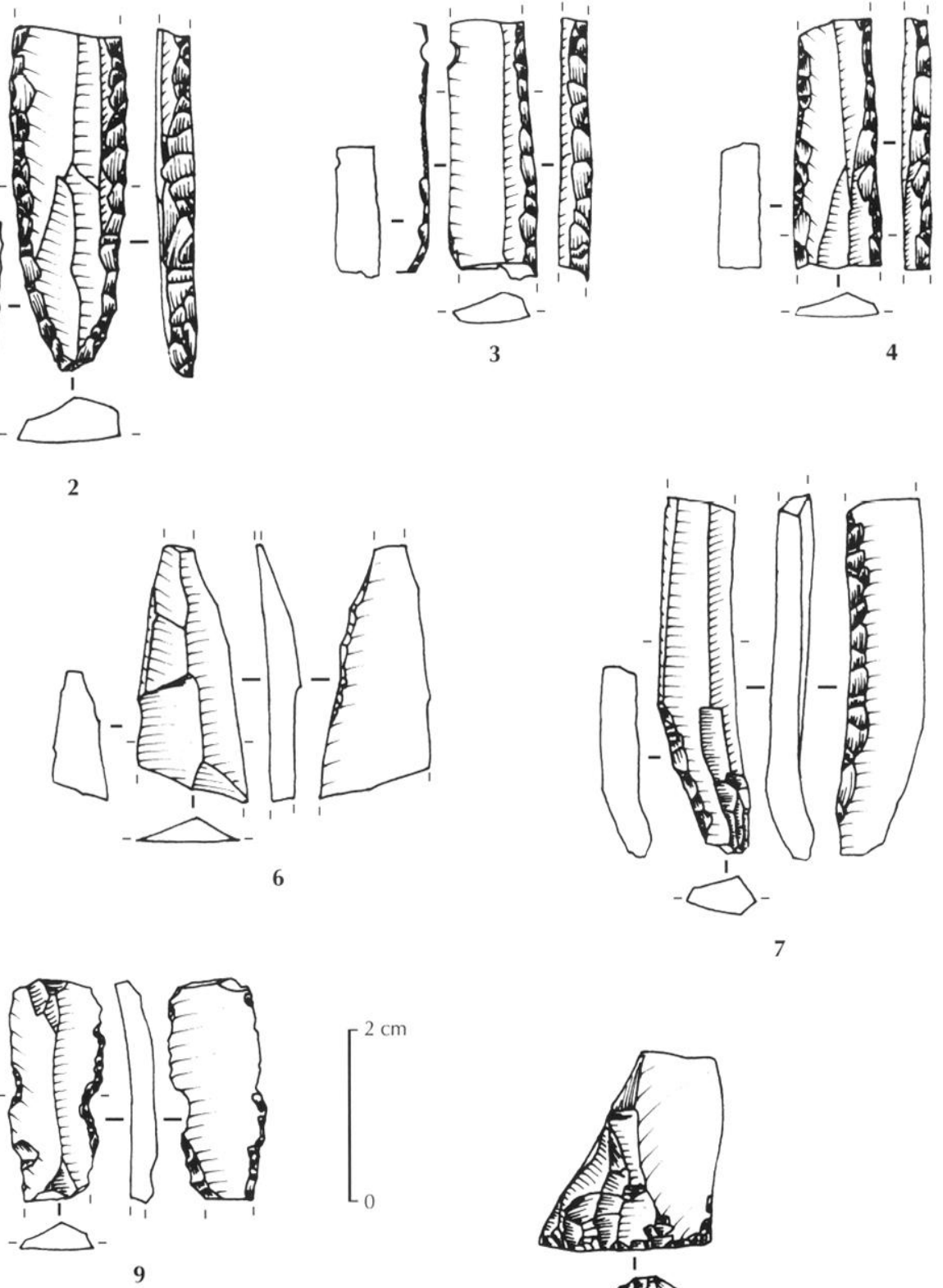

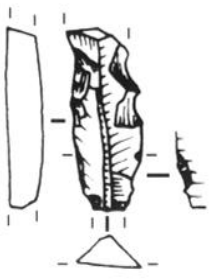

10

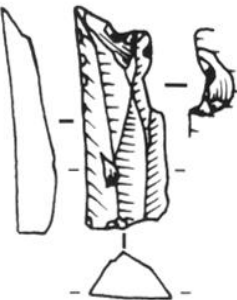

11
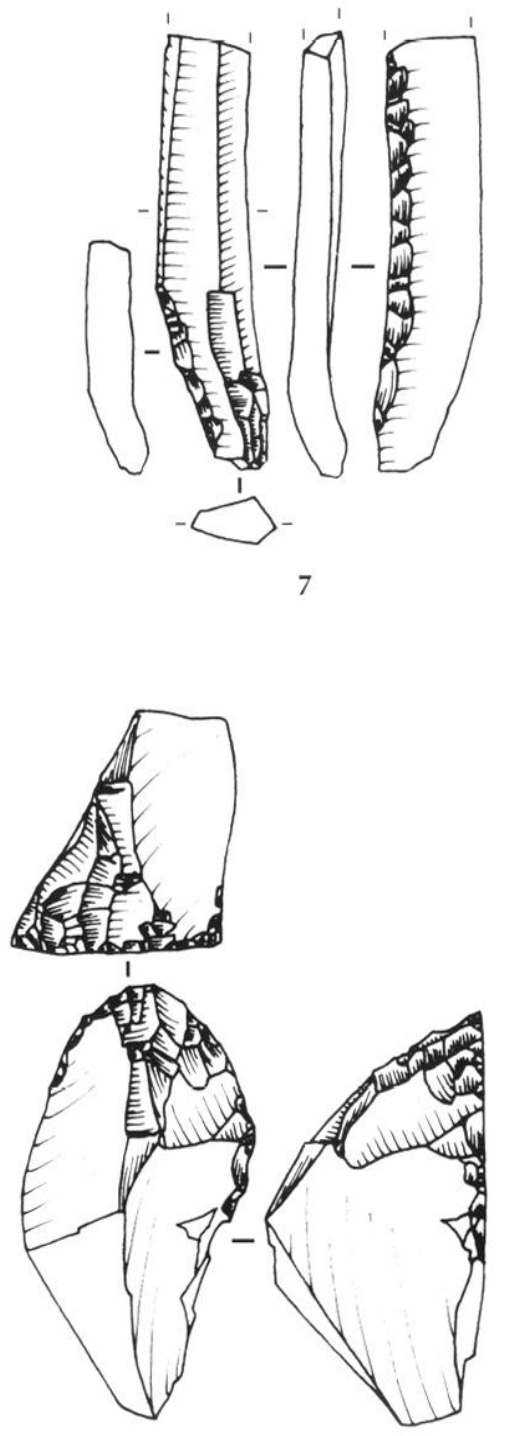

12

Fig. 26 - Outillage lamellaire du niveau 6: 1-5, lamelles de Font-Yves; 6, petite lamelle Dufour; 7, 8, grandes lamelles Dufour; 9, 11 , lamelles à encoche; 10, lamelle retouchée; 12, grattoir caréné sur lequel a été débité le support de la lamelle Inufour $n^{\circ} 8$ (dessins $I$. (Chiotti). 
$\left.\mathrm{n}^{\mathrm{os}} 7,8\right)$. Nous avons retrouvé un grattoir caréné en silex patiné blanc (fig. 26, $\mathrm{n}^{\circ}$ 12) dont les dimensions, la forme et la matière correspondent à la plus grande de ces deux lamelles ; c'est probablement le grattoir à partir duquel cette lamelle a été obtenue.

Les deux grandes lamelles Dufour portent une retouche inverse bien marquée, continue et régulière sur leur bord droit. Cette retouche est plus accentuée sur la partie mésiale, c'est-à-dire dans la partie la plus concave. Le bord gauche n'est retouché que dans sa partie proximale par une retouche directe.

Ces deux pièces sont atypiques dans le sens où elles n'entrent dans la définition d'aucun des deux sous-types de lamelles Dufour: Roc de Combe et Dufour (Demars, Laurent, 1989). Elles ont toutes les caractéristiques typologiques de lamelles Dufour, mais elles diffèrent par la technologie de leurs supports. Malgré leurs dimensions (et en particulier leur longueur), leurs supports (lamelles courbes et torses incontestablement issues de grattoirs carénés) ne nous permettent pas de les classer dans le sous-type Dufour, dont les supports sont de grandes lamelles à profil soit courbe, soit rectiligne. D'autre part, leurs grandes dimensions les excluent du sous-type Roc de Combe dont les supports sont bien des lamelles à profil torse, mais dont la longueur varie entre $15 \mathrm{~mm}$ et $20 \mathrm{~mm}$ (Demars, Laurent, 1989).

Cependant, les deux lamelles Dufour du niveau 6 se rapprochent plutôt du sous-type Roc de Combe pour plusieurs raisons. D'une part, les supports présentent le profil courbe et torse, et sont issus d'une même chaîne opératoire, seule leur longueur diffère. D'autre part, du point de vue typologique, elles portent une retouche identique qui, comme sur les petites lamelles Roc de Combe (Chiotti, 2000), a tendance à accentuer la concavité naturelle du bord droit.

En conclusion, l'outillage lamellaire du niveau 6 est constitué de deux types d'outils très différents.

Nous trouvons d'une part des pièces d'assez mauvaise facture, aménagées par quelques retouches, sur des supports qui n'ont pas été fabriqués pour cela, mais qui semblent avoir été choisis aléatoirement. Ces pièces sont conformes à la majeure partie de l'outillage de ce niveau.

D'autre part, il existe des outils bien élaborés (lamelles de Font-Yves et grandes lamelles Dufour) fabriqués sur des supports standardisés, soigneusement choisis : des lamelles de type D pour les lamelles de Font-Yves et des lamelles de grattoirs carénés ${ }^{6}$ pour les lamelles Dufour. Ces pièces bien élaborées sont assez exceptionnelles pour cette industrie. En effet, même parmi les outils de grandes dimensions, il est peu fréquent dans le niveau 6 d'avoir des outils d'une telle facture.

$$
* *
$$

Au terme de cette étude des lamelles de l'Aurignacien de l'abri Pataud, le premier caractère qui ressort concerne les quantités de lamelles produites. Il apparaît immédiatement qu'en règle générale les lamelles sont plus nombreuses dans l'Aurignacien évolué que dans l'Aurignacien ancien.

Le fait le plus important que nous avons pu mettre en évidence est la variabilité des techniques employées par les Aurignaciens pour obtenir des lamelles. Trois schémas principaux ont été identifiés tout au long de la séquence: une production à partir des grattoirs, une autre à partir des burins et une troisième à partir de nucléus prismatiques. De plus, à l'intérieur de ces trois schémas principaux, nous avons pu mettre en évidence des variations.

Pour les grattoirs, le schéma opératoire du débitage diffère selon qu'il s'agit de grattoirs carénés ou de grattoirs à museau épais (plus petits). Ces derniers permettent, de par les dimensions de leurs fronts, d'obtenir de petites lamelles sans aménagement de la longueur de la table de débitage. Au contraire, sur les grattoirs carénés, plus épais, cet aménagement est nécessaire, ce qui produit des lamelles de gabarit supérieur (type B), qui ne sont en fait que des sous-produits.

Dans l'Aurignacien ancien, nous avons pu différencier deux formes parmi les petites lamelles de type A obtenues par ces deux types de grattoirs-nucléus : d'une part, des lamelles courbes et torses et d'autre part, des lamelles courbes ou rectilignes, sans pouvoir préciser réellement lesquelles étaient recherchées. Cependant, selon l'étude d'autres sites, il semble que dans l'Aurignacien ancien ce soient les lamelles rectilignes qui étaient recherchées.

6. I.es deux lamelles servant de support aux grandes lamelles Dufour sont uniques dans cette industrie, voire même dans tout l'Aurignacien de l'abri Pataud. Elles proviennent incontestablement de grattoirs carénés, mais ne peuvent entrer ni dans le type A (longueur beaucoup trop importante), ni dans le type B (largeur trop faible et caractère volontaire incontestable, contrairement aux autres pièces de ce type, qui sont des sous-produits). 
Au contraire, dans l'Aurignacien évolué (au moins dans le niveau 8), les lamelles recherchées étaient incontestablement à la fois courbes et torses, et plus particulièrement celles qui sont déjetées à droite. Ce sont celles qui ont servi de supports aux lamelles Dufour du sous-type Roc-de-Combe. Pour les autres niveaux de l'Aurignacien évolué (7: Lower à 6), il y a également une majorité de lamelles courbes et torses, mais dont seulement une partie provient des grattoirs, puisqu'il y a un mélange avec les lamelles provenant des burins.

Contrairement aux grattoirs, la production des burins est homogène, qu'elle provienne des burins busqués, des burins carénés ou des burins des Vachons. Les lamelles produites (de type C) sont toujours des pièces courbes et torses qui sont, en moyenne, de dimensions un peu inférieures à celles provenant des grattoirs. Ce type de production est développé essentiellement dans l'Aurignacien évolué (à partir du niveau $7:$ Lower). Cependant, une chaîne opératoire similaire et complète a été rencontrée dans l'Aurignacien ancien du niveau 13, ce qui montre que, dès cette époque, la technique du burinnucléus était connue.

En définitive, pour l'Aurignacien évolué, les petites lamelles recherchées étaient bien des lamelles à la fois courbes et torses, qu'elles soient produites sur des grattoirs ou sur des burins.

Tout au long de la séquence aurignacienne de l'abri Pataud, c'est la production de ces petites lamelles qui domine très fortement, bien qu'une production de lamelles plus grandes, à partir de nucléus prismatiques (lamelles de type D), soit toujours présente. Comme celle des grattoirs, cette production présente des variations. Dans la plupart des niveaux, ces lamelles semblent être majoritairement des pièces intercalées dans des débitages laminaires, et donc plutôt involontaires. Il est également possible que dans certains niveaux une partie des lamelles dites opportunistes aient en fait la même origine.

Les nucléus à lamelles sont très rares dans toute la séquence, à l'exception du niveau 6 où ils sont bien représentés (surtout si on les compare au nombre de lamelles issues de cette production dans cette industrie). Ces nucléus peuvent être de deux types: soit de vrais nucléus à lamelles, soit des nucléus à lames réduits qui ont donné des lamelles à la fin de leur production.

La dernière partie de la présente étude concerne l'outillage lamellaire. Il est frappant de noter que, quel que soit le niveau considéré, ce petit outillage est extrêmement peu développé, surtout par rapport au nombre de lamelles produites. La majeure partie de cet outillage est constituée de pièces relativement peu retouchëes, réalisées sur des supports de tous types qui semblent choisis au hasard parmi les lamelles disponibles et qui ne présentent aucune standardisation. Les seules pièces de bonne facture et pour lesquelles les supports sont standardisés sont les lamelles Dufour et les lamelles de FontYves, et se situent quasiment toutes dans l'Aurignacien évolué.

Les lamelles Dufour sont réalisées sur les petites lamelles courbes et torses issues des grattoirs pour le niveau 8 (le plus riche), ou bien des grattoirs et/ou des burins pour les autres niveaux (très pauvres). Le déséquilibre très important entre le nombre de pièces produites et le nombre de pièces transformées nous pousse à proposer deux hypothèses: soit ces petites lamelles ont été utilisées brutes, soit la plupart des pièces transformées ont été emportées hors du site.

Les lamelles de Font-Yves ne sont présentes que dans le niveau 6. Elles sont également réalisées sur des supports standardisés, mais provenant cette fois-ci de nucléus prismatiques (lamelles de type D). La quantité de nucléus à lamelles, beaucoup plus importante dans ce niveau que dans les autres, est sans doute à mettre en relation avec la production de ce type d'outils. Cependant, et contrairement à ce qui est observé dans les autres niveaux et pour les autres types de lamelles, nous n'avons ici qu'un nombre très faible de produits par rapport aux nucléus. La matière première très facilement reconnaissable de certains nucléus nous permet même d'être certain que les lamelles qu'ils ont fournies ne sont pas présentes parmi les pièces retrouvées sur le site. Ceci peut se traduire de deux manières : soit les pièces ont été emportées (peut-être pour être transformées en lamelles de Font-Yves), soit les lamelles issues de ces nucléus ont été produites hors du site, ou dans un secteur non fouillé de l'abri.

En définitive, nous voudrions insister sur deux points qui nous paraissent essentiels : d'une part, la présence en parallèle de différentes chaînes opératoires ayant pour but l'obtention de lamelles et d'autre part, le rapport entre ces productions et leur utilisation comme supports d'outils, qui n'est pas toujours clair, ce qui traduit probablement le fait qu'une partie importante de ces lamelles devaient être utilisées brutes. 


\section{BIBLIOGRAPHIE}

Albry T., Detrain L., Kervizo B.

1995 : «Les niveaux intermédiaires entre

le Gravettien et le Solutréen de l'abri Casserole (Les Eyzies-de-Tayac): mise en évidence d'un mode de production original de microlithes et implications ". Bulletin de la Société préhistorique fransaise, 92, 3, p. 296-301.

BARION L., BouYssonit A., BouYssonit J. 1906 : "Grattoir caréné et ses dérivés à la Coumbâ Del Bouïtou (Corrèze) ". Revue de l'École d'Anthropologie, 16 , p. 401-411.

BON F.

2000 : La question de l'unité technique et économique de l'Aurignacien : réflexions. sur la variabilité des industries lithiques à partir de l'étude comparée de trois sites des Pyrénées francaises. La Tuto de Camalhot, Régismont-le-Haut et Brassempouy: Thèse de doctorat, univ. de Paris I - Panthéon-Sorbonne, $425 \mathrm{p}$.

BORIIES J.-G.

2000 : "La séquence aurignacienne de Caminade revisitéc: l'apport des raccords d'intérêt stratigraphique ". Paléo, 12, p. 387-407.

À paraître: "Objectifs et modalités de quelques productions lamellaires dans le sud-ouest de la France: Caminade, Roc de Combe, Corbiac Vignoble II et Le Piage ", in Actes du $X I V^{r}$ congrès de l'UISPP, colloque sur les productions lamellaires altribuées à l'Aurignacien : chaînes opératoires et perspectives techno-culturelles, Liège, sept. 2001.

BoRItes J.-G., LENoBt.: A.

2001 : Caminade (Sarlat, Dordogne). Document final de synthèse de fouille programmée, Service régional de l'archéologie d'Aquitaine, Bordeaux, $66 \mathrm{p}$.

2002 : «La " lamelle Caminade " : un nouvel outil lithique aurignacien?"
Bulletin de la Société préhistorique frangraise, 99, 4, p. 735-749.

Botyssonit A., Bol'yssonil J., BARIOON L. 1913 : « La station préhistorique de FontYves (Corrèze) ". Revue anthropologique, 23, 6, p. 218.

BOL YSSONIE J., SONAFTIIL-BORIDES D. IDE 1957 : "L'abri $n^{\circ} 2$ des Vachons, gisement aurignacien et périgordien, commune de Voulgézac (Charente) ", in Congrès préhistorique de France, $\mathrm{XV}^{\mathrm{c}}$ session, Poitiers, 1956, p. 271-309.

BRoOKS A. S.

1979 : The significance of variability in Paleolithic assemblages: An Aurignacian exumple from southwestern France. Thesis of Department of Anthropology, Harvard university, Cambridge, Massachussetts, 1057 p.

1995 : "L.Aurignacien de l'abri Pataud, nivcaux 6 à 11 ", in Brickl:R H. M. (dir.), Ie Paléolithique supénieur de l'abri Pataud (Dordogne) : les fouilles de H. L. Movius $\mathrm{fr}$. Paris, éd. de la Maison des sciences de l'homme (Documents d'archéologie française, 50), p. 167-222.

\section{ChIZZAN M.}

2001 : "Bladelet Production in the Aurignacian of La Ferrassie (Dordogne, France) ». Lithic Techno$\log ;, 26,1$, p. $16-28$.

Chicotiti I.

1999 : Les industries lithiques des niveraux aurignaciens de l'abri Pataud, Les Eyziesde-Tayac (Dordogne) : étude technologique et typologique. Thèse de doctorat du Muséum national d'histoire naturelle, Paris, 2 vol., 839 p.

2000 : "Lamelles Dufour et grattoirs aurignaciens (carénés et à museau) de la couche 8 de l'abri Pataud, Les Eyries-de-Tayac, Dordogne ". L'Anthropologie, 104, 2, p. 239-263.
À paraître : "La production lamellaire du niveau 8 (Aurignacien évolué) de l'abri Pataud (Les Eyzies-de-Tavac, Dordogne, France) ", in Actes du XIV congrès de l'UISPP, colloque sur les productions lamellaires attribuées à l'Aurignacien : chaînes opératoires el perspectives techno-culturelles, Liège, sept. 2001.

Chioter L., Lfo\% L. E., Nespoliet R., PotTtiak $C$.

À paraître : "Quelques exemples de stratégies d'approvisionnement dans l'Aurignacien et le Gravettien à l'abri Pataud (Dordogne) ", in Actes de la table ronde d'Aurillac: Matières premières lithiques. Inventaire, caractérisation et circulation durant la Préhistoire (Paléolithique, Mésolithique et Néolithique), en Europe occidentale, 20-22 juin 2002.

DALVOIS M.

1983 : "Burins usés de la grotte du (rest à Viry (Saône-et-Loire) ", in Congrès préhistorique de France, $\mathrm{XXI}^{\circ}$ session, Montauban-Cahors, sept. 1979, 2, p. $97-110$.

DEIPORTE: $\mathrm{H}$.

1968 : « L'abri du Facteur à Tursac (Dordogne) -I- Étude générale ". Gallia Préhistoire, XI, 1, p. 1-112.

DEMARS P.-Y.

1977 : Morphologie des outils caractéristiques des niveaux aurignaciens du bassin de Brive (Corrèze). Diplôme de l'École pratique des hautes études, Paris, $104 \mathrm{p}$.

1982 : "Les grattoirs carénés et à museau, les burins busqués et carénés, les pièces nucléiformes dans le bassin de Brive, approche stylistique ". Bulletin de la Société préhistorique francaise, 79, 10-12, p. 341-368.

1994 : L'économie du silex au Paléolithique supérieur dans le nord de l'Aquitaine. 
Analyse, synthèse et interprétations. Thèse de doctorat d'état, univ. de Bordeaux 1, 2 vol., 549 et 270 p.

DEMARS P.-Y., LAURENT P.

1989 : Types d'outils du Paléolithique supérieur en Europe. Paris, éd. du CNRS (Cahiers du Quaternaire, 14), $178 \mathrm{p}$.

\section{DJINDJIAN F.}

1993 : "L'Aurignacien du Périgord, une révision ", Préhistoire européenne, 3, p. 29-54.

ELoY L.

1946 : “Éclats de ravivage appartenant aux grattoirs carénés nucléiformes ou rabots des stations paléolithiques de Goyet-Mozet (province de Namur) ". Bulletin de la Société royale belge d'Anthropologie et de Préhistoire, 57, p. 192-195.

HAHN J., OWFN L. R.

1984 : «Débitage et utilisation de lames dans l'Aurignacien du Geissenklösterle, Jura Souabe ", in Préhistoire de la pierre taillée, économie du débitage laminaire. Paris, éd. du Cercle de recherches et d'études préhistoriques, 2 , p. 31-37.

INIZAN M.-L., REDLRON M., Roche H., TIXIER J.

1995 : Technologie de la pierre taillée. Meudon, éd. du Cercle de recherches et d'études préhistoriques, $4,197 \mathrm{p}$.

LF BRLN-RICALENS F.,

1996 : "L'occupation d'Hui à Beauville, Lot-ct-Garonne: activités domestiques en plein air à l'Aurignacien ancien ", in La Vie Préhistorique. Dijon/Paris, éd. Faton/Société préhistorique française, p. 80-85.

LF. BRUN-RICALENS F., BROU L.

Sous presse : « Burins carénés - nucléus à lamelles : identification d'une chaîne opératoire particulière à Thèmes (Yonne) et implications ", in: Fosse G., ThÉve.sin A., Le Paléolithique supérieur et le Mésolithique dans le nord- est de la France et les pays limitrophes. Actes de la table ronde de Valenciennes, 18-19 oct. 1997 (Cahiers de Préhistoire du Nord).

LLCAS G.

1997 : « Les lamelles Dufour du

Flageolet I (Bézenac, Dordogne) dans le contexte aurignacien ". Paléo, 9, p. 191-219.

1999 : «Production expérimentale de lamelles torses: approche préliminaire ". Bulletin de la Société préhistorique francaise, 96, p. 145-151.

2000 : Les industries lithiques du Flageolet I (Dordogne) : approche économique, technologique, fonctionnelle et analyse spatiale. Thèse de doctorat, univ. de Bordeaux 1, 2 vol., 307 et 295 p.

Marks A. E., Al..Mr:Il) F.

1996 : " The late Aurignacian and "Aurignacians" Elements in the Upper Palaeolithic of the Portuguese Estremadura, Portugal ", in Colloquium XI, The late Aurignacian., Actes du XIII" congrès de l'UISPP, Forli, sept. 1996. Forli, éd. ABACO, vol. 6, p. 11-21.

Movils H. L. JR.

1977 : Excavation of the abri Pataud, Les Eyzies (Dordogne): Stratigraphy. American School of Prehistoric Research, Peabody Museum, Harvard University, Cambridge, Massachussetts, $31,167 \mathrm{p}$.

Movic's H. L. JR. (ED.)

1975 : Excavation of the abri Pataud, Les Eyzies (Dordogne): Contributors. American School of Prehistoric Research, Peabody Museum, Harvard University, Cambridge, Massachussetts, 30, 305 p.

OTTE M.

1979 : Le Paléolithique supérieur ancien en Belgique. Bruxelles, Musées royaux d'art et d'histoire (Monographies d'archéologie nationale, 5), 684 p.

\section{Perpère: $\mathrm{M}$.}

1972a: "Les burins aurignaciens des Vachons (Charente) ", in Congrès pré- historique de France, XIX" session, Auvergne, 1969, p. 320-233.

1972b : "Remarques sur l'Aurignacien en Poitou-Charentes ". L'Anthropologie, 76, 5-6, p. 387-425.

PriroNy D.

1934 : "La Ferrassie, Moustérien Périgordien - Aurignacien ". Préhistoire, III, p. 1-92.

PRADEL. L.

1962 : «Du burin busqué au burin nucléiforme, formes de passage ". Bulletin de la Société préhistorique fransaise, I.IX, 9-10, p. 684-692.

1973 : «Traces d'usage sur les burins du Paléolithique supérieur ". Bulletin de la Société préhistorique franşaise, 70 , crsm 3, p. 90-96.

RI(GALI) J.-P.

1993 : «L'Aurignacien dans le sud-ouest de la France : bilan et perspectives ", in Aurignacien en Europe et au ProcheOrient. Actes du XII' congrès international des sciences préhistoriques et protohistoriques, UISPP, Bratislava, sept. 1991. Institut archéologique de l'Académie slovaque des sciences, p. 181-186.

\section{RONEN A.}

1964 : “Grattoirs carénés à encoche de l'Aurignacien ". Bulletin de la Société préhistorique fransaise, 61 , crsm 6 , p. 147-150.

\section{Schmider B., Prikpikr: M.}

1996 : "Production et utilisation de lamelles dans l'Aurignacien de la grotte du Renne, à Arcy-sur-Curc ", in Pautrat Y., Thevenis A. (éd.), Paléolithique supérieur et Épipaléolithique dans le Nord-Est de la France, Actes de la table ronde de Dijon, 7-8 oct. 1995. DRAC - SRA de Bourgogne, p. 4-10.

SONNFITLIE-BORIDES D. DE.

1970 : "Les industries aurignaciennes de l'abri Caminade est, commune de La Canéda (Dordogne) ". Quaternaria, Rome, 13, p. 77-131. 
SONNEvilite-Bordes D. DE, Mortureli B.

1955 : “L'abri Caminade, commune de La Canéda (Dordogne) ", Bulletin de la Société préhistorique fransaise, LII, 9-10, p. 608-619.
TIXIER J.

1963 : Typologie de l'Épipaléolithique du Maghreb. Paris, AMG (Mémoires du Centre de recherches anthropologiques préhistoriques et ethnographiques d'Alger, II), $212 \mathrm{p}$.
ZILHÃO J.

1997 : "Lapa da Anecrial ", in $O$ Paleolítico Superior da Estramadura portuguesa. Thèse de doctorat, univ. de Lisbonne, 1995, éd. Colibri, vol. II, p. 127-143. 